\title{
Dynamics of starbursting dwarf galaxies ${ }^{\star \star \star \star}$ III. A H I study of 18 nearby objects
}

\author{
Federico Lelli $^{1,2}$, Marc Verheijen ${ }^{1}$, and Filippo Fraternali ${ }^{3,1}$ \\ 1 Kapteyn Astronomical Institute, University of Groningen, Postbus 800, 9700 AV Groningen, The Netherlands \\ e-mail: lelli@astro.rug.nl \\ 2 Department of Astronomy, Case Western Reserve University, 10090 Euclid Avenue, Cleveland, OH 44106, USA \\ e-mail: federico.lelli@case.edu \\ 3 Department of Physics and Astronomy, University of Bologna, viale Berti Pichat 6/2, 40127 Bologna, Italy
}

Received 12 September 2013 / Accepted 24 April 2014

\section{ABSTRACT}

\begin{abstract}
We investigate the dynamics of starbursting dwarf galaxies, using both new and archival H I observations. We consider 18 nearby galaxies that have been resolved into single stars by HST observations, providing their star formation history and total stellar mass. We find that 9 objects have a regularly rotating H I disk, 7 have a kinematically disturbed H I disk, and 2 show unsettled H I distributions. Two galaxies (NGC 5253 and UGC 6456) show a velocity gradient along the minor axis of the HI disk, which we interpret as strong radial motions. For galaxies with a regularly rotating disk we derive rotation curves, while for galaxies with a kinematically disturbed disk, we estimate the rotation velocities in their outer parts. We derive baryonic fractions within about 3 optical scale lengths and find that, on average, baryons constitute at least $30 \%$ of the total mass. Despite the star formation having injected $\sim 10^{56}$ ergs in the ISM in the past $\sim 500 \mathrm{Myr}$, these starbursting dwarfs have both baryonic and gas fractions similar to those of typical dwarf irregulars, suggesting that they did not eject a large amount of gas out of their potential wells.
\end{abstract}

Key words. galaxies: dwarf - galaxies: starburst - galaxies: kinematics and dynamics - galaxies: evolution - dark matter

\section{Introduction}

Starburst activity is thought to strongly affect the evolution of dwarf galaxies. Both observations and theoretical models suggest that massive star formation can alter the morphology and kinematics of the gas in dwarf galaxies (e.g., Mac Low \& Ferrara 1999; Cannon et al. 2011), as well as their chemical properties (e.g., Recchi et al. 2004; Romano et al. 2006). Moreover, models of galaxy formation in a $\Lambda$ cold dark matter $(\Lambda \mathrm{CDM})$ cosmology require strong feedback from star formation to explain several observational facts, such as i) the existence of bulgeless disk galaxies by removing low angular-momentum gas from the galaxy center (e.g., Governato et al. 2010; Brook et al. 2011); ii) the "cored" DM profiles observed in dwarfs by flattening the presumed central "cusps" (e.g., Oh et al. 2011a; Governato et al. 2012); iii) the slope of the baryonic Tully-Fisher relation by reducing the baryonic fraction in galaxies (e.g., McGaugh 2012; Stringer et al. 2012); and iv) the number density of lowluminosity galaxies by suppressing star formation in low-mass DM halos (e.g., Okamoto et al. 2010; Sawala et al. 2013). Detailed dynamical studies of nearby starbursting dwarfs are necessary to determine the actual efficiency of these processes.

Starbursting dwarfs can be identified by i) their blue colors and high surface brightness, such as the blue compact dwarfs (BCDs; e.g., Gil de Paz et al. 2003); ii) their strong emission-lines, such as the HII-galaxies (e.g.,

\footnotetext{
* Appendices are available in electronic form at http: //www . aanda. org

$\star \star$ H I datacubes (FITS files) are only available at the CDS via anonymous ftp to cdsarc.u-strasbg. fr $(130.79 .128 .5)$ or via http: //cdsarc.u-strasbg.fr/viz-bin/qcat?]/A+A/566/A71
}

Terlevich et al. 1991; Taylor et al. 1995); and iii) their peculiar morphologies, such as the "amorphous dwarfs" (e.g., Gallagher \& Hunter 1987; Marlowe et al. 1999). Hereafter, we refer to any starbursting dwarf as a BCD.

To date, detailed studies of the HI kinematics of BCDs have been focused either on individual galaxies (e.g., Viallefond \& Thuan 1983; Hunter et al. 1996; Wilcots \& Miller 1998; Matthews \& Uson 2008) or on small galaxy samples with four to five objects (e.g., van Zee et al. 1998, 2001; Thuan et al. 2004; Ramya et al. 2011). These studies show that some BCDs have regularly rotating HI disks (e.g., van Zee et al. 1998, 2001), whereas others have complex H I kinematics (e.g., Cannon et al. 2004; Kobulnicky \& Skillman 2008). The relative fraction of BCDs with ordered or disturbed HI kinematics remains unclear, as does the possible relation between the gas kinematics and the starburst. The DM content of starbursting dwarfs is also poorly constrained. Elson et al. $(2010,2013)$ argue that the BCDs NGC 1705 and NGC 2915 are dominated by DM at all radii (see also Meurer et al. 1996, 1998). In contrast, Walter et al. (1997) and Johnson et al. (2012) studied the starbursting dwarfs II Zw 33 and NGC 1569, respectively, and conclude that there is no need for DM to explain their inner kinematics.

To clarify these issues, we considered a sample of 18 starbursting dwarfs, for which we collected both new and archival H I observations. We selected objects that have been resolved into single stars by the Hubble Space Telescope (HST), providing a direct estimate of the recent star-formation history ( $\mathrm{SFH}$ ) and of the total stellar mass (e.g., Annibali et al. 2003). The latter information allows us to break the "disk-halo degeneracy" (van Albada \& Sancisi 1986) and to estimate baryonic fractions. In Lelli et al. (2012a,b), we presented our results for two show-case galaxies: I Zw 18 and UGC 4483. For both objects, 
Table 1. Galaxy sample.

\begin{tabular}{|c|c|c|c|c|c|c|c|c|c|c|c|}
\hline Name & $\begin{array}{l}\text { Alternative } \\
\text { name }\end{array}$ & $\begin{array}{c}\text { Dist } \\
(\mathrm{Mpc})\end{array}$ & $\begin{array}{c}M_{*} \\
\left(10^{7} M_{\odot}\right) \\
\end{array}$ & $M_{\mathrm{R}}$ & $\begin{array}{c}M_{*} / L_{\mathrm{R}} \\
\left(M_{\odot} / L_{\odot}\right) \\
\end{array}$ & $b$ & $\begin{array}{c}S F R_{\mathrm{p}} \\
\left(10^{-3} M_{\odot} \mathrm{yr}^{-1}\right) \\
\end{array}$ & $\begin{array}{c}t_{\mathrm{p}} \\
(\mathrm{Myr}) \\
\end{array}$ & $\begin{array}{c}\Sigma_{\mathrm{SFR}}\left(t_{\mathrm{p}}\right) \\
\left(10^{-3} M_{\odot} \mathrm{yr}^{-1} \mathrm{kpc}^{-2}\right)\end{array}$ & $12+\log (\mathrm{O} / \mathrm{H})$ & Ref. \\
\hline NGC 625 & ESO 297-G005 & $3.9 \pm 0.4$ & $26 \pm 10$ & $-17.25 \pm 0.24$ & $>0.6$ & $3.0 \pm 0.1$ & $86 \pm 20$ & $820 \pm 180$ & $2.5 \pm 0.6$ & $8.08 \pm 0.12$ & $\mathrm{a}, \mathrm{g}, 1$ \\
\hline NGC 1569 & UGC 3056 & $3.4 \pm 0.2$ & $70 \pm 7$ & $-17.14 \pm 0.25$ & $1.7 \pm 0.2$ & $21 \pm 1$ & $240 \pm 10$ & $40 \pm 10$ & $8.5 \pm 0.3$ & $8.19 \pm 0.02$ & $\mathrm{a}, \mathrm{h}, \mathrm{m}$ \\
\hline NGC 1705 & ESO 158-G013 & $5.1 \pm 0.6$ & $>20$ & $-16.35 \pm 0.26$ & $>1$ & $\sim 6$ & $314 \pm 78$ & $\sim 3$ & $44 \pm 11$ & $8.21 \pm 0.05$ & $\mathrm{~b}, \mathrm{i}, \mathrm{l}$ \\
\hline NGC 2366 & UGC 3851 & $3.2 \pm 0.4$ & $26 \pm 3$ & $-16.64 \pm 0.27$ & $1.0 \pm 0.1$ & $5.6 \pm 0.4$ & $160 \pm 10$ & $450 \pm 50$ & $2.6 \pm 0.2$ & $7.91 \pm 0.05$ & $\mathrm{a}, \mathrm{h}, \mathrm{l}$ \\
\hline NGC 4068 & UGC 7047 & $4.3 \pm 0.1$ & $22 \pm 3$ & $-15.67 \pm 0.05$ & $2.0 \pm 0.3$ & $4.7 \pm 0.3$ & $42 \pm 3$ & $360 \pm 40$ & $4.5 \pm 0.3$ & $\ldots$ & $\mathrm{a}, \mathrm{h}$ \\
\hline NGC 4163 & UGC 7199 & $3.0 \pm 0.1$ & $10 \pm 3$ & $-14.81 \pm 0.10$ & $2.0 \pm 0.6$ & $2.9 \pm 0.6$ & $12 \pm 3$ & $450 \pm 50$ & $3.8 \pm 0.9$ & $7.56 \pm 0.14$ & $\mathrm{a}, \mathrm{h}, \mathrm{l}$ \\
\hline NGC 4214 & UGC 7278 & $2.7 \pm 0.2$ & $>28$ & $-17.77 \pm 0.24$ & $>0.4$ & $3.1 \pm 0.9$ & $130 \pm 40$ & $450 \pm 50$ & $8.5 \pm 2.6$ & $8.22 \pm 0.05$ & $\mathrm{a}, \mathrm{h}, \mathrm{l}$ \\
\hline NGC 4449 & UGC 7592 & $4.2 \pm 0.5$ & $210 \pm 35$ & $-18.88 \pm 0.26$ & $1.0 \pm 0.2$ & $6.0 \pm 0.5$ & $970 \pm 70$ & $5 \pm 3$ & $28 \pm 2$ & $8.26 \pm 0.09$ & $\mathrm{a}, \mathrm{h}, \mathrm{l}$ \\
\hline NGC 5253 & Haro 10 & $3.5 \pm 0.4$ & $154 \pm 21$ & $-17.61 \pm 0.27$ & $2.4 \pm 0.3$ & $9.0 \pm 0.9$ & $400 \pm 40$ & $450 \pm 50$ & $29 \pm 3$ & $8.12 \pm 0.05$ & $\mathrm{a}, \mathrm{g}, \mathrm{k}$ \\
\hline NGC 6789 & UGC 11425 & $3.6 \pm 0.2$ & $7 \pm 2$ & $-15.09 \pm 0.14$ & $1.1 \pm 0.3$ & $3.8 \pm 1.3$ & $15 \pm 5$ & $565 \pm 65$ & $9.7 \pm 3.2$ & $\ldots$ & $\mathrm{a}, \mathrm{i}$ \\
\hline UGC 4483 & $\ldots$ & $3.2 \pm 0.2$ & $1.0 \pm 0.2$ & $-12.97 \pm 0.19$ & $1.1 \pm 0.3$ & $14 \pm 3$ & $11 \pm 2$ & $565 \pm 65$ & $8.8 \pm 3.5$ & $7.56 \pm 0.03$ & $\mathrm{a}, \mathrm{i}, 1$ \\
\hline UGC 6456 & VII Zw 403 & $4.3 \pm 0.1$ & $5 \pm 2$ & $-14.41 \pm 0.05$ & $1.5 \pm 0.6$ & $7.6 \pm 1.1$ & $23 \pm 3$ & $16 \pm 8$ & $5.1 \pm 0.7$ & $7.69 \pm 0.01$ & $\mathrm{a}, \mathrm{j}, \mathrm{n}$ \\
\hline UGC 6541 & Mrk 178 & $4.2 \pm 0.2$ & $>0.8$ & $-14.61 \pm 0.10$ & $>0.2$ & $\sim 3$ & $\sim 3$ & $\ldots$ & $1.2 \pm 0.6$ & $7.82 \pm 0.06$ & $\mathrm{c}, \mathrm{j}, 1$ \\
\hline UGC 9128 & DDO 187 & $2.2 \pm 0.1$ & $1.3 \pm 0.2$ & $-12.82 \pm 0.12$ & $1.6 \pm 0.2$ & $6.3 \pm 1.4$ & $5 \pm 1$ & $150 \pm 50$ & $4.4 \pm 0.9$ & $7.75 \pm 0.05$ & $\mathrm{a}, \mathrm{h}, \mathrm{l}$ \\
\hline UGCA 290 & Arp 211 & $6.7 \pm 0.4$ & $>1.0$ & $-14.09 \pm 0.18$ & $>0.4$ & $\sim 3$ & $42 \pm 15$ & $\sim 15$ & $16 \pm 6$ & $\ldots$ & $\mathrm{d}, \mathrm{i}$ \\
\hline I Zw 18 & Mrk 116 & $18.2 \pm 1.4$ & $>1.7$ & $-14.99 \pm 0.26$ & $>0.3$ & $\sim 30$ & $\sim 100$ & $\sim 10$ & $\sim 127$ & $7.20 \pm 0.01$ & $e, j, k$ \\
\hline I Zw 36 & Mrk 209 & $5.9 \pm 0.5$ & $>0.8$ & $-14.88 \pm 0.23$ & $>0.1$ & $\sim 7$ & $\sim 25$ & $\ldots$ & $\sim 9.8$ & $7.77 \pm 0.01$ & $\mathrm{f}, \mathrm{i}, \mathrm{k}$ \\
\hline SBS $1415+437$ & $\ldots$ & $13.6 \pm 1.4$ & $17 \pm 3$ & $-15.90 \pm 0.25$ & $1.3 \pm 0.2$ & $12 \pm 2$ & $150 \pm 10$ & $450 \pm 50$ & $8.3 \pm 0.5$ & $7.62 \pm 0.03$ & $\mathrm{a}, \mathrm{i}, \mathrm{o}$ \\
\hline
\end{tabular}

Notes. Distances are derived from the TRGB. Stellar masses are calculated integrating the SFHs and assuming i) a Salpeter IMF from 0.1 to $100 M_{\odot}$; and ii) a gas-recycling efficiency of $30 \%$. The birthrate parameter $b$ is defined as $b=S F R_{\mathrm{p}} / \overline{S F R}_{0-6}$, where $\mathrm{SFR}_{\mathrm{p}}$ is the peak $\mathrm{SFR}$ over the past $1 \mathrm{Gyr}$ and $\overline{\mathrm{SFR}}_{0-6}$ is the mean SFR over the past $6 \mathrm{Gyr}$ (see McQuinn et al. 2010a). $t_{\mathrm{p}}$ is the look-back time at $\mathrm{SFR}$. $\Sigma_{\mathrm{SFR}}\left(t_{\mathrm{p}}\right)$ is the SFR surface density at $t_{\mathrm{p}}$, calculated as $S F R_{\mathrm{p}} /\left(\pi R_{\mathrm{opt}}^{2}\right.$ ) where $R_{\mathrm{opt}}$ is the optical radius (see Table 3 ). The last column provides references for the HST studies of the resolved stellar populations, the integrated photometry, and the ionized gas metallicity, respectively.

References. (a) McQuinn et al. (2010a); (b) Annibali et al. (2003); (c) Schulte-Ladbeck et al. (2000); (d) Crone et al. (2002); (e) Annibali et al. (2013); (f) Schulte-Ladbeck et al. (2001); (g) Lauberts \& Valentijn (1989); (h) Swaters \& Balcells (2002); (i) Gil de Paz et al. (2003); (j) Papaderos et al. (2002); (k) Izotov \& Thuan (1999); (1) Berg et al. (2012); (m) Kobulnicky \& Skillman (1997); (n) Thuan \& Izotov (2005); (o) Guseva et al. (2003).

we showed that i) the H I gas forms a compact, rotating disk; ii) the rotation curve rises steeply in the inner parts and flattens in the outer regions; and iii) old stars and atomic gas are dynamically important, since they constitute at least $\sim 30 \%$ of the total dynamical mass within the last measured point of the rotation curve. Here we present a dynamical study of the remaining 16 objects.

\section{The sample}

Table 1 summarizes the main properties of our sample of starbursting dwarfs. For these 18 galaxies, the studies of the resolved stellar populations provide i) the galaxy distance from the tip of the red giant branch (TRGB); ii) the spatial distribution of the different stellar populations; iii) the recent SFH (ages $\lesssim 1 \mathrm{Gyr}$ ) by modeling the color-magnitude diagrams (CMDs); iv) the energy produced during the burst by supernovae and stellar winds; and v) the stellar mass in young and old stars. For 13 objects, we adopt the SFHs derived by McQuinn et al. (2010a) using archival HST images. The remaining 5 objects (I Zw 18, I Zw 36, NGC 1705, UGC 6541, and UGCA 290) have not been studied by McQuinn et al. (2010a) because the HST observations have a relatively shallow photometric depth $(\lesssim 1 \mathrm{mag}$ below the TRGB). We use the SFHs derived by other authors (Annibali et al. 2003, 2013; Crone et al. 2002; Schulte-Ladbeck et al. 2000, 2001), although they are more uncertain due to the limited photometric depth. Note that all these 18 objects are well-defined starburst galaxies, as their star-formation rates (SFRs) show an increase in the recent $\mathrm{SFH}$ by a factor $\gtrsim 3$ with respect to the past, average SFR. The sample covers a broad range in luminosities $\left(-19 \lesssim M_{R} \lesssim-13\right)$, stellar masses $\left(10^{7} \lesssim M_{*} / M_{\odot} \lesssim 10^{9}\right)$, and metallicities $\left(0.3 \lesssim Z / Z_{\odot} \lesssim 0.03\right)$.

For all these galaxies, we collected both new and archival H I data. We obtained new H I observations for SBS 1415+437 and UGCA 290 using the Jansky Very Large Array (VLA, during its upgrade period), and of NGC 6789 using the Westerbork Synthesis Radio Telescope (WSRT). We analyzed raw data from the VLA archive for I Zw 18 (Lelli et al. 2012a), UGC 4483 (Lelli et al. 2012b), UGC 6456 and NGC 625 (this work). The HI datacubes of NGC 1705 (Elson et al. 2013) and NGC 5253 (López-Sánchez et al. 2012) were kindly provided by Ed Elson and Angel R. Lopez-Sanchez, respectively. For the remaining 9 galaxies, we used HI cubes from 3 public surveys: WHISP (Swaters et al. 2002), THINGS (Walter et al. 2008), and LITTLE-THINGS (Hunter et al. 2012). For 4 galaxies (NGC 2366, NGC 4163, NGC 4214, and UGC 9128), H I cubes are available from both WHISP and THINGS/LITTLETHINGS; we used the VLA data as they have higher spatial resolution than the WSRT observations.

\section{Data reduction and analysis}

\subsection{HI data}

In the following, we outline the main steps of the H I data analysis. We first describe the new $21 \mathrm{~cm}$-line observations and the data reduction. For the latter, we followed procedures similar to 
F. Lelli et al.: Dynamics of starbursting dwarf galaxies. III.

Table 2. New $21 \mathrm{~cm}$-line observations.

\begin{tabular}{lccccc}
\hline \hline Galaxy & Array & Project & Observing dates & Time on source & Calibrators \\
\hline \multirow{2}{*}{ UGCA 290 } & VLA/B & 10C-200 & 12, 26, 28 Mar. 2011 & $7.6 \mathrm{~h}$ & 3C 286, 1227+365 \\
& VLA/C & $12 \mathrm{~A}-246$ & 25, 27, 28 Apr. 2012 & $4.6 \mathrm{~h}$ & 3C 286, 1227+365 \\
& VLA/D & 11B-075 & 2, 13 Nov. 2011 & $0.28 \mathrm{~h}$ & 2C 295, 1227+365 \\
\multirow{5}{*}{ SBS 1415+345 } & VLA/B & 10C-200 & 4, 27 Mar., and 8 Apr. 2011 & $7.6 \mathrm{~h}$ & 3C 286, 1400+621 \\
& VLA/C & 12A-246 & 26, 28, 30 Apr. 2012 & $4.6 \mathrm{~h}$ & 3C 286, 1400+621 \\
& VLA/D & 11B-075 & 6, 9 Oct. 2011 & $0.28 \mathrm{~h}$ & 3C 295, 1400+621 \\
NGC 6789 & WSRT & R11A007 & 20 May 2011 & 12 h & 3C 286, 3C 48 \\
\hline
\end{tabular}

Lelli et al. (2012a,b) and refer to these papers for further details. For the existing H I datacubes, we refer to the original papers (see Table B.1). Finally, we describe the derivation of total H I maps and velocity fields.

NGC 6789 was observed in May 2011 with the WSRT in a standard $12 \mathrm{~h}$ session. The correlator was used in dualpolarization mode, with a total bandwidth of $10 \mathrm{MHz}$ and 1024 spectral channels, providing a velocity resolution of $\sim 2.5 \mathrm{~km} \mathrm{~s}^{-1}$. SBS $1415+437$ and UGCA 290 were observed with the B, C, and D arrays of the VLA between March 2011 and April 2012. The correlator was used in dual-polarization WIDAR mode with a total bandwidth of $2.0 \mathrm{MHz}$ and 256 spectral line channels, providing a velocity resolution of $\sim 1.9 \mathrm{~km} \mathrm{~s}^{-1}$. Between 20 Sept. 2011 and 3 Dec. 2011, the VLA correlator back-end, by mistake, integrated for only $1 \mathrm{sec}$ per record, thus the D-array observations have a time on source of only $\sim 16$ min instead of the expected $2 \mathrm{~h}$. The new H I observations are summarized in Table 2. We also reduced archival VLA observations of NGC 625 and UGC 6456.

The raw UV data were flagged, calibrated, and combined using the AIPS package and following standard procedures. We Fourier-transformed the UV data using a robust weighting technique (Briggs 1995). After various trials, we chose the value of the robust parameter $\mathfrak{R}$ (either $-1,-0.5$, or 0 ) that minimizes sidelobes and wings in the beam profile. After the Fourier transform, we continued the data analysis using the Groningen Imaging Processing SYstem (GIPSY; van der Hulst et al. 1992). The channel maps were continuum-subtracted using line-free channels and then cleaned (Högbom 1974) down to $0.3 \sigma$ using a mask to define the search areas.

A detailed study of the H I kinematics requires a combination of spatial resolution, spectral resolution, and sensitivity that varies from object to object, depending both on the quality of the H I observations and on the intrinsic properties of the galaxy (e.g., angular size, rotation velocity, mean H I column density). We used the following approach. For every galaxy, we first analyzed the H I datacube at the highest spatial and spectral resolutions available. This cube is typically obtained using $\mathfrak{R} \simeq 0$ and has relatively low column-density sensitivity, but the synthesized beam profile is close to a Gaussian and does not have the broad wings that are typical for natural-weighted UV-data. Then, we smoothed the cube both in the image plane and in velocity using various Gaussian tapers, until we found the optimal compromise between resolution and sensitivity. The properties of both original and final datacubes are summarized in Table B.1. The spatial and spectral resolutions range between $5^{\prime \prime}$ to $30^{\prime \prime}$ and 5 to $10 \mathrm{~km} \mathrm{~s}^{-1}$, respectively.

Total H I maps were obtained by summing the masked channel maps. The masks were constructed by first smoothing the datacubes in the image plane to $30^{\prime \prime}$ or $60^{\prime \prime}$ (depending on the angular extent of the galaxy) and in velocity to $\sim 10$ or $\sim 20 \mathrm{~km} \mathrm{~s}^{-1}$ (depending on the H I line-width of the galaxy), and subsequently clipping the channel maps at $3 \sigma_{\mathrm{s}}\left(\sigma_{\mathrm{s}}\right.$ is the rms noise in the smoothed cube). A pseudo- $3 \sigma$ contour in the total H I map was calculated following Verheijen \& Sancisi (2001). The H I datacube of NGC 1569 is strongly affected by Galactic emission that we have interactively blotted out; the resulting H I map is rather uncertain. Velocity fields (VFs) were derived by fitting a Gaussian function to the H I line profiles. Fitted Gaussians with a peak intensity less than $3 \sigma$ were discarded. For most galaxies, the HI line profiles are very broad and asymmetric, thus the VFs only provide a rough description of the galaxy kinematics. As a consequence, our kinematical analysis is mostly based on channel maps, Position-Velocity (PV) diagrams, and 3-dimensional (3D) disk models.

\subsection{Optical data}

In order to compare the relative $\mathrm{HI}$ and stellar distributions of the BCDs, we collected optical images via the NASA/IPAC Extragalactic Database $\left(\mathrm{NED}^{1}\right)$. When available, we used $R$-band images, otherwise we used $V$-band ones. The images come from the following studies: Kuchinski et al. (2000), Gil de Paz et al. (2003), Taylor et al. (2005), Hunter \& Elmegreen (2006), and Meurer et al. (2006). In I Zw 18, the nebular emission line dominates the optical morphology, thus we have also used the $\mathrm{H} \alpha$-subtracted, $R$-band image from Papaderos et al. (2002).

The images were analyzed as follows. We determined the sky level by masking the sources in the frame and fitting a $2 \mathrm{D}$ polynomial to the masked image. Then, we created sky-subtracted images and isophotal maps, using the calibration parameters provided in the original papers. To improve the signal-to-noise ratio in the outer regions, the isophotal maps were pixel-averaged with a $3 \times 3$ box; this preserves the resolution of the images as the pixel sizes were typically $\sim 3$ times smaller than the seeing.

The images were interactively fitted with ellipses to determine the optical center $\left(\alpha_{\mathrm{opt}}, \beta_{\mathrm{opt}}\right)$, position angle $\left(\mathrm{PA}_{\mathrm{opt}}\right)$, and ellipticity $\epsilon_{\mathrm{opt}}$. Foreground stars and scattered light in the frames were masked out by hand. Since the inner isophotes of BCDs are usually irregular due to the starburst, we estimated $\left(\alpha_{\text {opt }}, \beta_{\text {opt }}\right)$, $\mathrm{PA}_{\mathrm{opt}}$, and $\epsilon_{\mathrm{opt}}$ by taking the mean value over the outer isophotes. We also estimated the optical inclination $i_{\text {opt }}$ using the formula

$\cos ^{2}\left(i_{\text {opt }}\right)=\frac{\left(1-\epsilon_{\text {opt }}\right)^{2}-q_{0}^{2}}{1-q_{0}^{2}}$

1 The NASA/IPAC Extragalactic Database is operated by the Jet Propulsion Laboratory, California Institute of Technology, under contract with the National Aeronautics and Space Administration. 


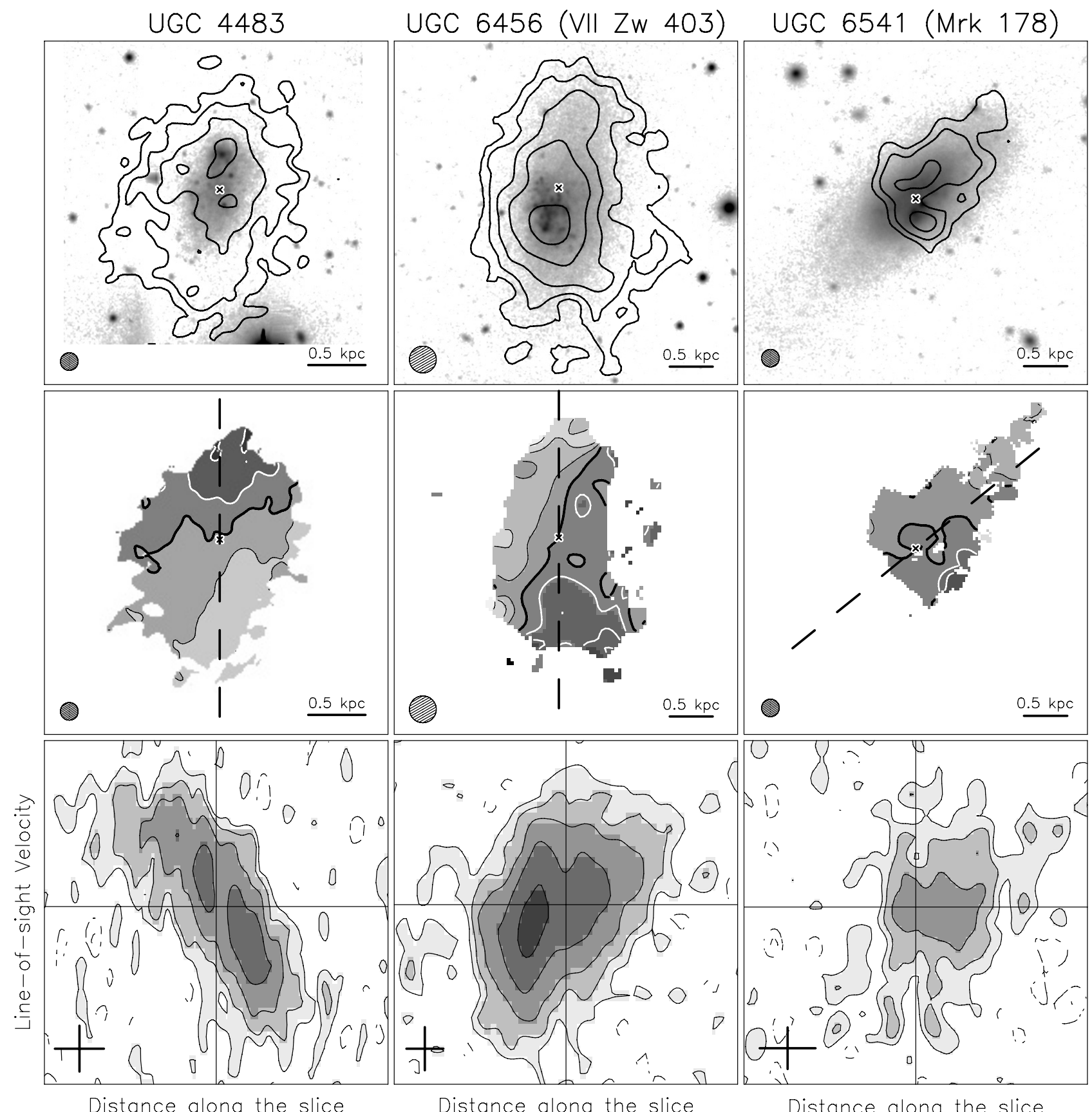

Fig. 1. Examples of BCDs with different H I distribution and kinematics: UGC 4483 (left) has a regularly rotating H I disk, UGC 6456 (center) has a kinematically disturbed H I disk, and UGC 6456 (right) has an unsettled H I distribution. Top: optical image superimposed with the total H I map (contours). The contours are the same as in Appendix C. The cross shows the optical center; the circle shows the HI beam. Middle: H I velocity field. Light and dark shading indicate approaching and receding velocities, respectively. The thick, black line shows the systemic velocity. The isovelocity contours are the same as in Appendix C. The dashed line indicates the HI major axis. The cross and the circle are the same as in the top panel. Bottom: position-velocity diagrams taken along the major axis. The cross corresponds to $0.5 \mathrm{kpc} \times 10 \mathrm{~km} \mathrm{~s}{ }^{-1}$. The horizontal and vertical lines indicate the systemic velocity and the galaxy center, respectively.

where $q_{0}$ is the intrinsic thickness of an oblate stellar disk. We assumed $q_{0}=0.3$, as indicated by statistical studies of the ellipticities of dwarf galaxies (Binggeli \& Popescu 1995; Sánchez-Janssen et al. 2010). The orientation parameters of different isophotes often show relatively large variations with radii, that we used to estimate the uncertainties in $\mathrm{PA}_{\text {opt }}$ and $i_{\text {opt }}\left(\sim 2^{\circ}\right.$ to $\sim 6^{\circ}$, except for NGC 4214 that is very close to face-on). The resulting orientation parameters are provided in Table B.2. The sky-subtracted images and isophotal maps are presented in Appendix C.
For 11 galaxies in our sample, Swaters \& Balcells (2002) and Papaderos et al. (2002) derived $R$-band luminosity profiles that were used to estimate the scale length $R_{\mathrm{d}}$ and the central surface brightness $\mu_{0}$ of the old stellar component by fitting an exponential law to their outer parts. For the remaining 7 objects, we derived luminosity profiles both for the whole galaxy and for the approaching/receding sides separately, by azimuthally averaging the $R$-band images over concentric ellipses. We did not correct the profiles for internal extinction, as BCDs are usually metalpoor (see Table 1) and the dust content is expected to be low. 
F. Lelli et al.: Dynamics of starbursting dwarf galaxies. III.

Table 3. Structural properties of the H I disk.

\begin{tabular}{lccccc}
\hline \hline Name & $\begin{array}{c}M_{\mathrm{HI}} \\
\left(10^{7} M_{\odot}\right)\end{array}$ & $\begin{array}{c}R_{\mathrm{HI}} \\
(\mathrm{kpc})\end{array}$ & $\begin{array}{c}R_{\mathrm{opt}} \\
(\mathrm{kpc})\end{array}$ & $R_{\mathrm{HI}} / R_{\mathrm{opt}}$ & Ref. \\
\hline \multicolumn{5}{c}{ Galaxies with } & regularly rotating H I disk \\
NGC 1705 & $11.1 \pm 2.9$ & 2.1 & 1.5 & 1.4 & $\mathrm{a}$ \\
NGC 2366 & $62 \pm 17$ & 6.8 & 4.4 & 1.5 & $\mathrm{~b}$ \\
NGC 4068 & $14.9 \pm 1.6$ & 3.1 & 1.8 & 1.7 & $\mathrm{~b}$ \\
NGC 4214 & $43 \pm 8$ & 5.5 & 2.2 & 2.5 & $\mathrm{~b}$ \\
NGC 6789 & $1.8 \pm 0.3$ & 1.0 & 0.7 & 1.4 & $\mathrm{a}$ \\
UGC 4483 & $2.9 \pm 0.5$ & 1.4 & 0.6 & 2.3 & $\mathrm{a}$ \\
I Zw 18 & $21 \pm 3$ & 3.3 & 0.5 & 6.6 & $\mathrm{c}$ \\
I Zw 36 & $6.7 \pm 1.3$ & 1.9 & 0.9 & 2.1 & $\mathrm{a}$ \\
SBS 1415+437 & $20.1 \pm 4.6$ & 4.3 & 2.4 & 1.8 & $\mathrm{a}$ \\
Galaxies with a kinematically & disturbed HI disk & & \\
NGC 625 & $9.7 \pm 2.2$ & 2.6 & 3.3 & 0.8 & $\mathrm{a}$ \\
NGC 1569 & $29.1 \pm 4.5$ & 3.9 & 3.0 & 1.3 & $\mathrm{~b}$ \\
NGC 4163 & $1.5 \pm 0.2$ & 1.1 & 1.0 & 1.1 & $\mathrm{~b}$ \\
NGC 4449 & $300 \pm 77$ & 8.9 & 3.3 & 2.7 & $\mathrm{~b}$ \\
NGC 5253 & $13.8 \pm 3.4$ & 3.1 & 2.1 & 1.5 & $\mathrm{a}$ \\
UGC 6456 & $4.5 \pm 0.5$ & 1.8 & 1.2 & 1.5 & $\mathrm{c}$ \\
UGC 9128 & $1.3 \pm 0.2$ & 0.9 & 0.6 & 1.5 & $\mathrm{~b}$ \\
Galaxies with unsettled HI distribution & & & \\
UGC 6541 & $1.2 \pm 0.2$ & $\ldots$ & 0.9 & $\ldots$ & $\mathrm{c}$ \\
UGCA 290 & $1.4 \pm 0.2$ & $\ldots$ & 0.9 & $\ldots$ & $\mathrm{a}$ \\
\hline
\end{tabular}

Notes. The H I radius $R_{\mathrm{HI}}$ is defined as the radius where the H I surface density profile reaches $1 M_{\odot} \mathrm{pc}^{-2}$. The optical radius $R_{\mathrm{opt}}$ is defined as $3.2 R_{\mathrm{d}}$, where $R_{\mathrm{d}}$ is the exponential scale length. The last column provides references for $R_{\mathrm{d}}$.

References. a) this work, b) Swaters \& Balcells (2002), c) Papaderos et al. (2002).

Finally, we estimated the central surface brightness $\mu_{0}$ and scale length $R_{\mathrm{d}}$ of the old stellar component by fitting an exponential law to the side of the disk that is least affected by the starburst (as in Lelli et al. 2012b for UGC 4483).

\section{HI distribution and kinematics}

In the following, we discuss the distribution and kinematics of the high-column-density gas associated with the stellar component of BCDs. In Appendix A we discuss individual galaxies in detail and compare our results to previous studies, while in Appendix $\mathrm{C}$ we present an atlas with optical images, isophotal maps, total H I maps, H I surface density profiles, H I velocity fields, and PV-diagrams.

Based on the HI morphology and kinematics, we classify starbursting dwarfs into three main families:

\section{- BCDs with a regularly rotating H I disk ( $\sim 50 \%)$.}

The PV-diagram along the H I major axis shows a regular velocity gradient (see Fig. 1, left). The VF displays a pattern that is typical of a rotating disk, although minor asymmetries caused by non-circular motions may be present. In some cases, the kinematical center and PA may not coincide with the optical ones. For these galaxies, we derive rotation curves (see Sect. 5).
- BCDs with a kinematically disturbed H I disk ( 40\%).

The H I distribution resembles a disk, but the PV-diagrams and the VF are irregular and asymmetric (see Fig. 1, center). For these galaxies, it is not possible to derive a reliable rotation curve, but we obtain rough estimates of the kinematical parameters and of the rotation velocity in the outer parts (see Sect. 6).

- BCDs with unsettled H I distributions ( 10\%).

Both the HI distribution and kinematics are irregular and asymmetric (see Fig. 1, right), and they are inconsistent with a rotating disk.

For the 16 galaxies with a H I disk, we derived H I surface density profiles by azimuthally averaging the H I maps over ellipses with a width of 1 beam-size. We assumed the orientation parameters defined by the HI kinematics (see Table B.2). Following Swaters et al. (2002), we calculated i) the HI radius $R_{\mathrm{HI}}$, defined as the radius where the HI surface density profile (corrected for inclination) reaches $1 M_{\odot} \mathrm{pc}^{-2}$; and ii) the optical radius $R_{\text {opt }}$, defined as 3.2 scale lengths $R_{\mathrm{d}}$. The latter definition allows us to compare the sizes of galaxies with different central surface brightnesses: for an exponential disk with $\mu_{0}(B)=$ $21.65 \mathrm{mag} \operatorname{arcsec}^{-2}$ (Freeman 1970), $R_{\mathrm{opt}}=3.2 R_{\mathrm{d}}$ corresponds to the isophotal radius $R_{25}$. In our sample of $\mathrm{BCDs}$, the ratio $R_{\mathrm{HI}} / R_{\text {opt }}$ ranges from $\sim 1$ to $\sim 3$ (see Table 3 ), which is typical for gas-rich spirals and irregulars (Irrs; e.g., Verheijen \& Sancisi 2001 ). The only exception is I Zw 18 with $R_{\mathrm{HI}} / R_{\mathrm{opt}} \simeq 6.6$, as the HI distribution extends towards a secondary stellar body (see Appendix C and Lelli et al. 2012a). Excluding I Zw 18, the mean value of $R_{\mathrm{HI}} / R_{\mathrm{opt}}$ is $1.7 \pm 0.5$, in close agreement with the values found by Swaters et al. (2002) for 73 gas-rich dwarfs $(1.8 \pm 0.8)$ and by Broeils \& Rhee (1997) for 108 gas-rich spirals $(1.7 \pm 0.5)$.

Previous H I studies have shown that BCDs have centrallyconcentrated H I distributions with higher surface densities than typical Irrs (Taylor et al. 1994; van Zee et al. 1998, 2001; Simpson \& Gottesman 2000). We confirm this result for a larger sample of galaxies. In Fig. 2 (left), we compare the mean H I surface density profile of late-type dwarfs obtained by Swaters et al. (2002, averaging over 73 objects) with the mean HI surface density profile of our starbursting dwarfs (averaging over the 16 objects with a H I disk). Note that the sample of Swaters et al. (2002) contains a few BCDs that are also part of our sample, but it is fully dominated by typical, non-starbursting Irrs. Figure 2 (left) shows that BCDs, on average, have central H I surface densities a factor of $\sim 2$ higher than typical Irrs. In several cases, the central, azimuthally averaged H I surface densities can be as high as $\sim 20 M_{\odot} \mathrm{pc}^{-2}$ (see e.g., NGC 1569 and NGC 1705 in Appendix C). The actual peak H I column densities can reach even higher values, up to $\sim 50-100 M_{\odot} \mathrm{pc}^{-2}$ in I Zw 18 at a linear resolution of $\sim 200$ pc (Lelli et al. 2012a).

When comparing the H I surface densities of different galaxies, a possible concern is the different linear resolutions (in kpc) of the $21 \mathrm{~cm}$-line observations. The $16 \mathrm{H} \mathrm{I}$ cubes used here have linear resolutions ranging from $\sim 0.2$ to $\sim 0.7 \mathrm{kpc}$ (see Table B.1), while the 73 cubes used by Swaters et al. (2002) have linear resolutions ranging from $\sim 0.4$ to $\sim 2 \mathrm{kpc}$ (apart for 13 cases where the linear resolution is $\gtrsim 2 \mathrm{kpc}$ ). To quantify the effect of beam smearing, we derived HI surface density profiles by smoothing our data to $30^{\prime \prime}$ (as in Swaters et al. 2002), and checked that the distribution of the ratio $R_{\mathrm{HI}}$ /beam-size is comparable for the two samples. We found that the azimuthally averaged $\mathrm{HI}$ surface density of BCDs decreases by only $\sim 20 \%$ in the inner parts. Thus, we conclude that the difference between the mean 

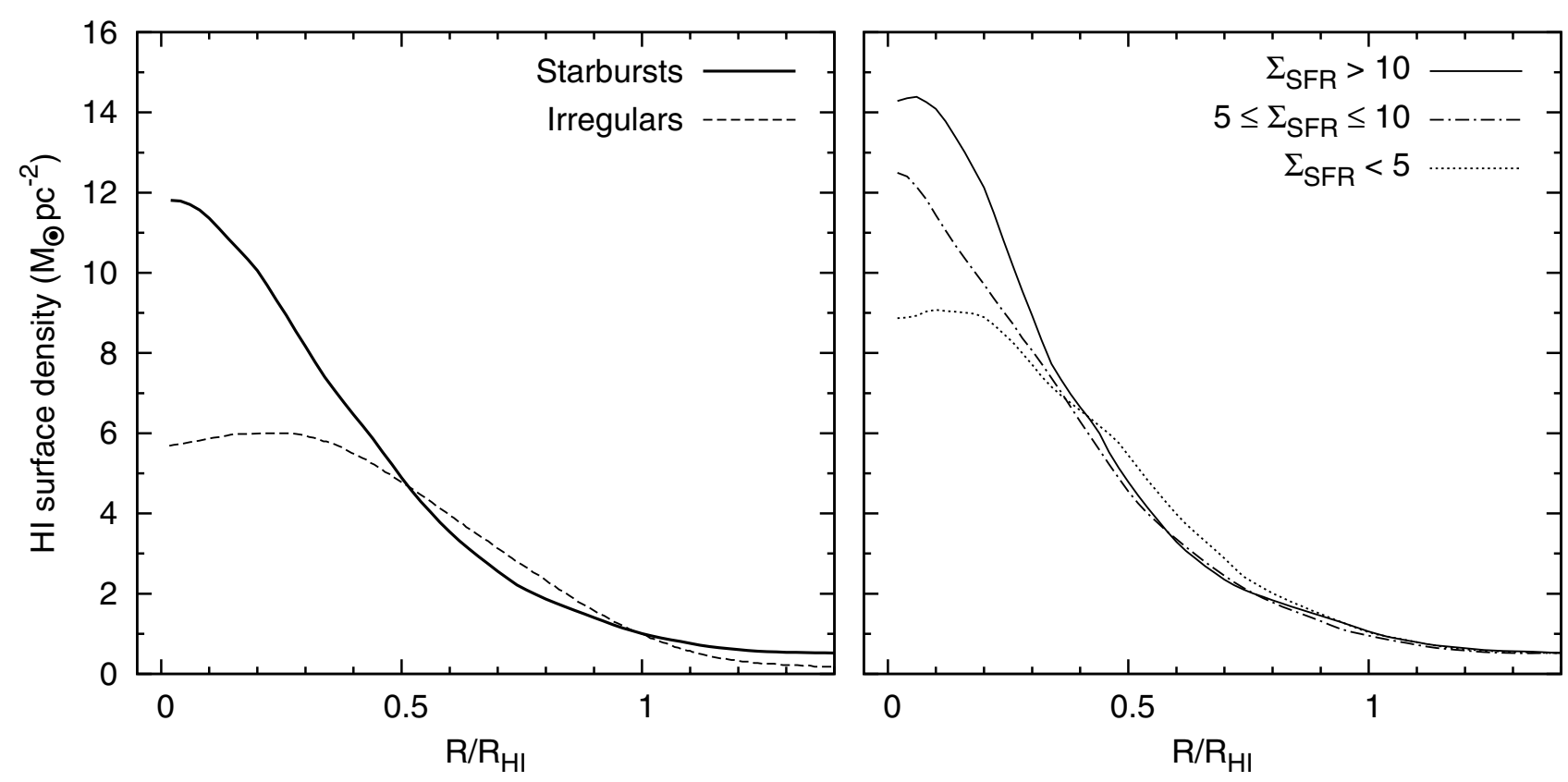

Fig. 2. Left: average H I surface density profile for starbursting dwarfs (solid line) and for typical irregulars (dashed line, from Swaters et al. 2002). Right: average H I surface density profiles for starbursting dwarfs binned by $\Sigma_{\text {SFR }}$ (in units of $10^{-3} M_{\odot} \mathrm{yr}^{-1} \mathrm{kpc}^{-2}$ ) as indicated in the legend to the top-right.

H I surface density profile of BCDs and Irrs is not due to observational effects.

Figure 2 (right) shows the mean HI surface density profiles of BCDs binned by SFR surface density $\Sigma_{\mathrm{SFR}}=$ $S F R_{\mathrm{p}} /\left(\pi R_{\mathrm{opt}}^{2}\right)$ (see Table 1). We used three $\Sigma_{\mathrm{SFR}}$ bins (in units of $\left.10^{-3} M_{\odot} \mathrm{yr}^{-1} \mathrm{kpc}^{-2}\right): \Sigma_{\mathrm{SFR}}<5$ (five objects), $5 \leq \Sigma_{\mathrm{SFR}} \leq$ 10 (seven objects), and $\Sigma_{\mathrm{SFR}}>10$ (four objects). It is clear that galaxies with higher values of $\Sigma_{\mathrm{SFR}}$ have higher central H I surface densities, as one may expect from the Kennicutt-Schmidt law (e.g., Kennicutt 1998). We also derived mean H I surface density profiles binned by the value of the birthrate parameter $b$ (see Table 1), but found no strong relation between $b$ and the shape of the HI surface density profile. The relation between star formation, gas surface density, and internal dynamics is further investigated and discussed in Lelli et al. (2014).

Finally, we compare the overall H I kinematics of BCDs and typical Irrs. A detailed comparison between the rotation curves of BCDs and Irrs is presented in Lelli et al. (2014). Irrs generally have regularly rotating H I disks. For example, Swaters et al. (2009) studied the H I kinematics of 69 late-type dwarfs and could derive rotation curves for 62 objects ( $90 \%)$. In contrast, for our sample of 18 starbursting dwarfs, rotation curves could be derived for only $50 \%$ of the galaxies, as the other objects have either a kinematically disturbed $\mathrm{HI}$ disk or an unsettled H I distribution. This suggests that complex H I kinematics are much more common in BCDs than in typical Irrs. This may be related to the starburst trigger (e.g., interactions/mergers or disk instabilities) and/or be a consequence of feedback from supernovae (SN) and stellar winds.

\section{Galaxies with a regularly rotating $\mathrm{HI}$ disk}

\subsection{Derivation of the rotation curves}

Nine galaxies in our sample $(50 \%)$ have a regularly rotating HI disk: NGC 1705, NGC 2366, NGC 4068, NGC 4214, NGC 6789, UGC 4483, I Zw 18, I Zw 36, and SBS 1415+437.
For these objects, we derived rotation curves following a procedure similar to Swaters et al. (2009). As a first step, we obtained initial estimates of the geometrical parameters and of the rotation curve by fitting a tilted-ring model to the VF (Begeman 1987). These initial estimates were then used as input to build a 3D kinematic model, which was subsequently corrected by trial and error to obtain a model-cube that matches the observations. The model-cubes consider the spatial and spectral resolution of the observations, the observed gas distribution, the velocity dispersion, the disk thickness, and possibly non-circular motions. For I Zw 18 and UGC 4483, the derivation of the rotation curve is described in detail in Lelli et al. (2012a,b). In the following, we briefly describe the derivation of the rotation curves for the remaining galaxies.

The VF was fitted with a tilted-ring model using a ring width of one beam and weighting every point by $\cos ^{2}(\theta)$, where $\theta$ is the azimuthal angle in the plane of the galaxy from the major axis. The parameters of the fit are the kinematical center $\left(\alpha_{\text {kin }}, \delta_{\text {kin }}\right)$, the systemic velocity $V_{\text {sys }}$, the position angle $\mathrm{PA}_{\text {kin }}$, the inclination $i_{\text {kin }}$, and the rotation velocity $V_{\text {rot }}$. We first left all the parameters free, and determined $\left(\alpha_{\text {kin }}, \delta_{\text {kin }}\right)$ and $V_{\text {sys }}$ by taking the average value within the innermost rings. Then, we fixed $\left(\alpha_{\mathrm{kin}}, \delta_{\mathrm{kin}}\right)$ and $V_{\text {sys }}$, and determined $\mathrm{PA}_{\text {kin }}$ and $i_{\text {kin }}$. Finally, we determined $V_{\text {rot }}$ at every radius, keeping all the other parameters fixed. The errors on $V_{\text {rot }}$ have been estimated by considering the differences in the rotation curves derived from the approaching and receding sides separately (see e.g., Lelli et al. 2012b).

The 3D disk models were built assuming that the $\mathrm{HI}$ kinematics is axisymmetric while the H I distribution is clumpy, i.e., the surface density varies with position as in the observed H I map (see Lelli et al. 2012a,b for details). We also assumed that i) the H I disk has an exponential vertical distribution with a constant scale height $z_{0}$ of $\sim 100 \mathrm{pc}$; and ii) the velocity dispersion $\sigma_{\mathrm{HI}}$ is constant with radius. The actual value of $z_{0}$ does not affect our results, since kinematic models with different scale heights (up to $1 \mathrm{kpc}$ ) show no significant differences in their channel maps and PV-diagrams. For the mean velocity 
F. Lelli et al.: Dynamics of starbursting dwarf galaxies. III.
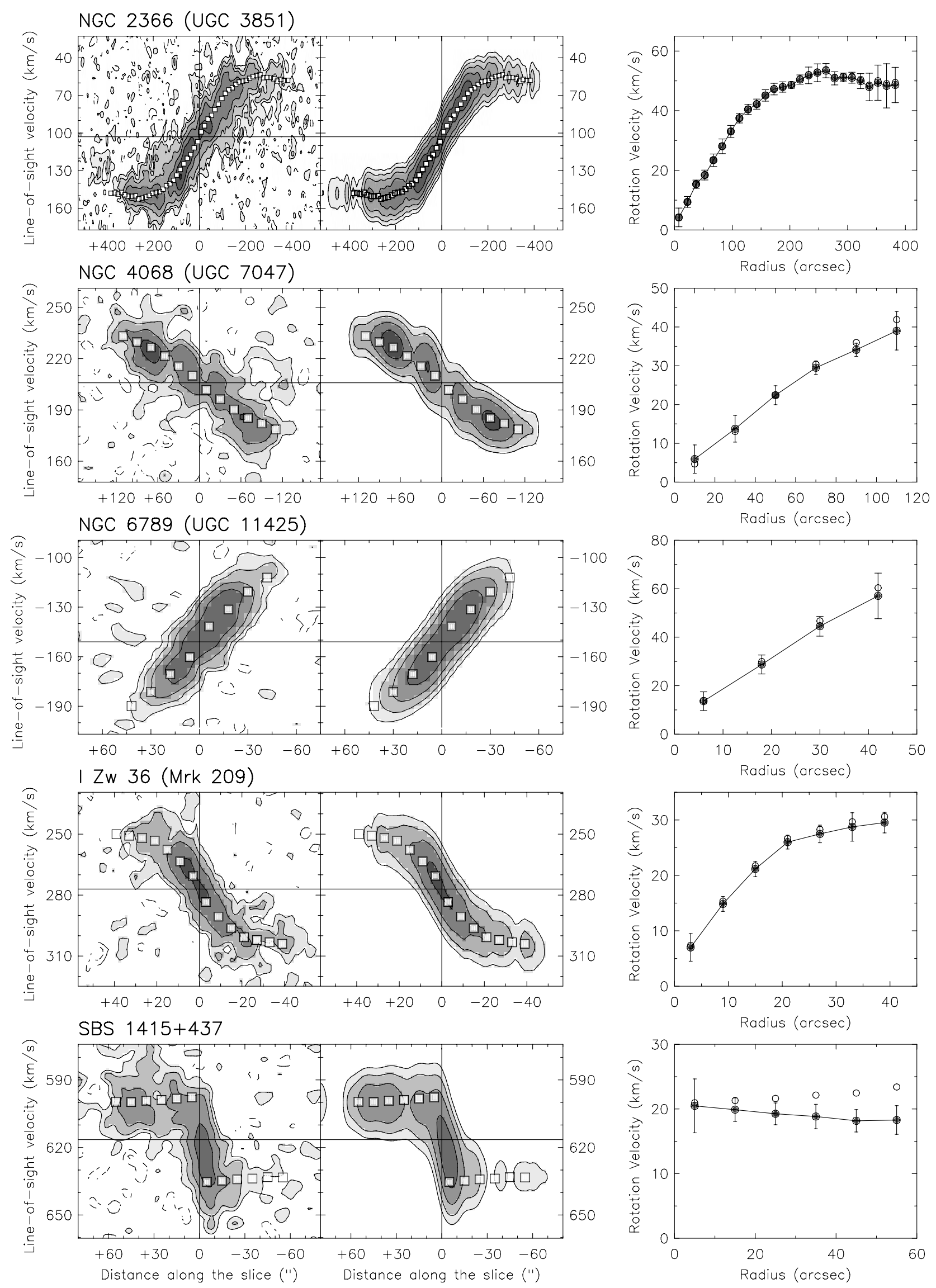

Fig. 3. Kinematical analysis of five BCDs with a regularly rotating H I disk. Left: PV-diagrams obtained along the disk major axis from the observed cube and the model-cube. Contours are at -1.5 (dashed), 1.5, 3, $6,12 \sigma$. The squares show the rotation curve used to build the models, projected along the line of sight. Right: observed rotation curve (filled circles) and asymmetric-drift-corrected rotation curve (open circles). See Sect. 5 for details. 

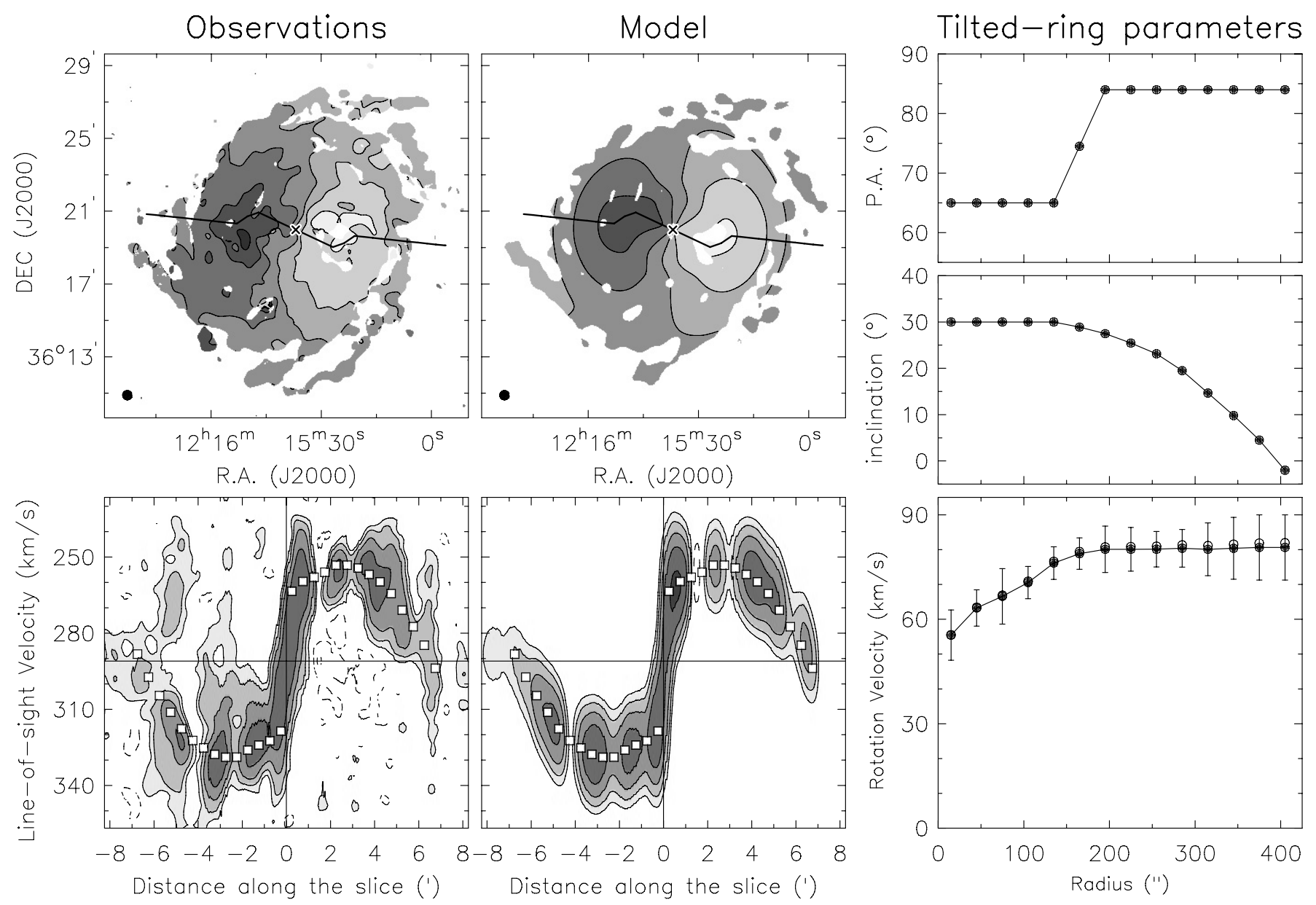

Fig. 4. The warped galaxy NGC 4214. Left: velocity fields (top) and PV-diagrams (bottom) obtained from the observed cube and the model-cube. In the velocity fields, the contours range from 257 to $337 \mathrm{~km} \mathrm{~s}^{-1}$ with steps of $16 \mathrm{~km} \mathrm{~s}^{-1}$; the thick line shows the line of nodes; the circle to the bottom-left shows the beam. In the PV-diagrams, the contours are at -1.5 (dashed), 1.5, 3, $6,12 \sigma$; the squares show the rotation curve projected along the line of sight. Right: tilted-ring parameters for the disk model. The open circles show the asymmetric-drift-corrected rotation curve. See Sect. 5 for details.

Table 4. Parameters for the asymmetric-drift correction.

\begin{tabular}{lccccc}
\hline \hline Galaxy & Funct. & $\begin{array}{c}\Sigma_{0, \mathrm{HI}} \\
\left(M_{\odot} \mathrm{pc}^{-2}\right)\end{array}$ & $\begin{array}{c}R_{0, \mathrm{HI}} \\
(\mathrm{kpc})\end{array}$ & $\begin{array}{c}s \\
(\mathrm{kpc})\end{array}$ & $\begin{array}{c}\sigma_{\mathrm{HI}} \\
\left(\mathrm{km} \mathrm{s}^{-1}\right)\end{array}$ \\
\hline NGC 1705 $\left(R<80^{\prime \prime}\right)$ & Exp & 24.0 & 0.7 & $\ldots$ & 12 \\
NGC 1705 $\left(R>80^{\prime \prime}\right)$ & Exp & 1.5 & 2.8 & $\ldots$ & 12 \\
NGC 2366 & Gauss & 9.1 & 0.8 & 6.31 & 10 \\
NGC 4068 & Gauss & 9.0 & 1.0 & 0.92 & 8 \\
NGC 4214 & Exp & 13.6 & 2.5 & $\ldots$ & 10 \\
NGC 6789 & Gauss & 16.4 & 0.0 & 0.37 & 8 \\
I Zw 36 & Exp & 11.9 & 0.7 & $\ldots$ & 9 \\
SBS 1415+357 & Exp & 11.8 & 1.4 & $\ldots$ & 9 \\
\hline
\end{tabular}

dispersion $\sigma_{\mathrm{HI}}$, we estimated values between 8 and $12 \mathrm{~km} \mathrm{~s}^{-1}$ (see Table 4) by comparing several PV-diagrams obtained from models and observations; values higher than $12 \mathrm{~km} \mathrm{~s}^{-1}$ are generally ruled out. Possible variations of $\sigma_{\mathrm{HI}}$ with radius of $\sim 2-3 \mathrm{~km} \mathrm{~s}^{-1}$ would generally produce no appreciable differences in the model-cubes. An exception is NGC 4214, which seems to have $\sigma_{\mathrm{HI}} \simeq 15 \mathrm{~km} \mathrm{~s}^{-1}$ for $R \lesssim 1^{\prime}$.

Figures 3-5 compare PV-diagrams obtained from both the observations and the models along the disk major axis. We generally found a good agreement between models and observations when increasing the values of $V_{\text {rot }}$ derived with the tiltedring fit to the VF by $\sim 2$ to $3 \mathrm{~km} \mathrm{~s}^{-1}$ at the innermost radii. Exceptions are NGC 4214 and SBS 1415+437, as their inner velocity-points require a correction of $\sim 10 \mathrm{~km} \mathrm{~s}^{-1}$ due to severe beam-smearing effects. For NGC 1705, the corrections to the velocity-points are even larger ( $\sim 20$ to $\left.30 \mathrm{~km} \mathrm{~s}^{-1}\right)$ because the H I disk appears close to edge-on in the outer parts and, thus, the VF only provides a poor representation of the rotation velocities. We also used PV-diagrams at the full resolution (see Table B.1) to further check that beam-smearing effects do not significantly affect our rotation curves. In two cases (NGC 4068 and SBS 1415+437), the 3D disk models also suggest that the position of the kinematical center should be shifted by about one beam with respect to the results of the tilted-ring fit to the VF.

The observed PV-diagram of NGC 2366 shows H I emission close to the systemic velocity that cannot be reproduced by our disk model (see Fig. 3). This is likely due to extra-planar H I emission that is rotating at a lower velocity than the disk (a socalled "lagging H I halo", see e.g., Fraternali et al. 2002), as it is observed in several nearby spiral galaxies (e.g., Sancisi et al. 2008). The modeling of a lagging H I halo is beyond the scope of this paper.

The galaxies NGC 4214 and NGC 1705 deserve special attention, as their H I disks are close to face-on in the inner parts 
F. Lelli et al.: Dynamics of starbursting dwarf galaxies. III.
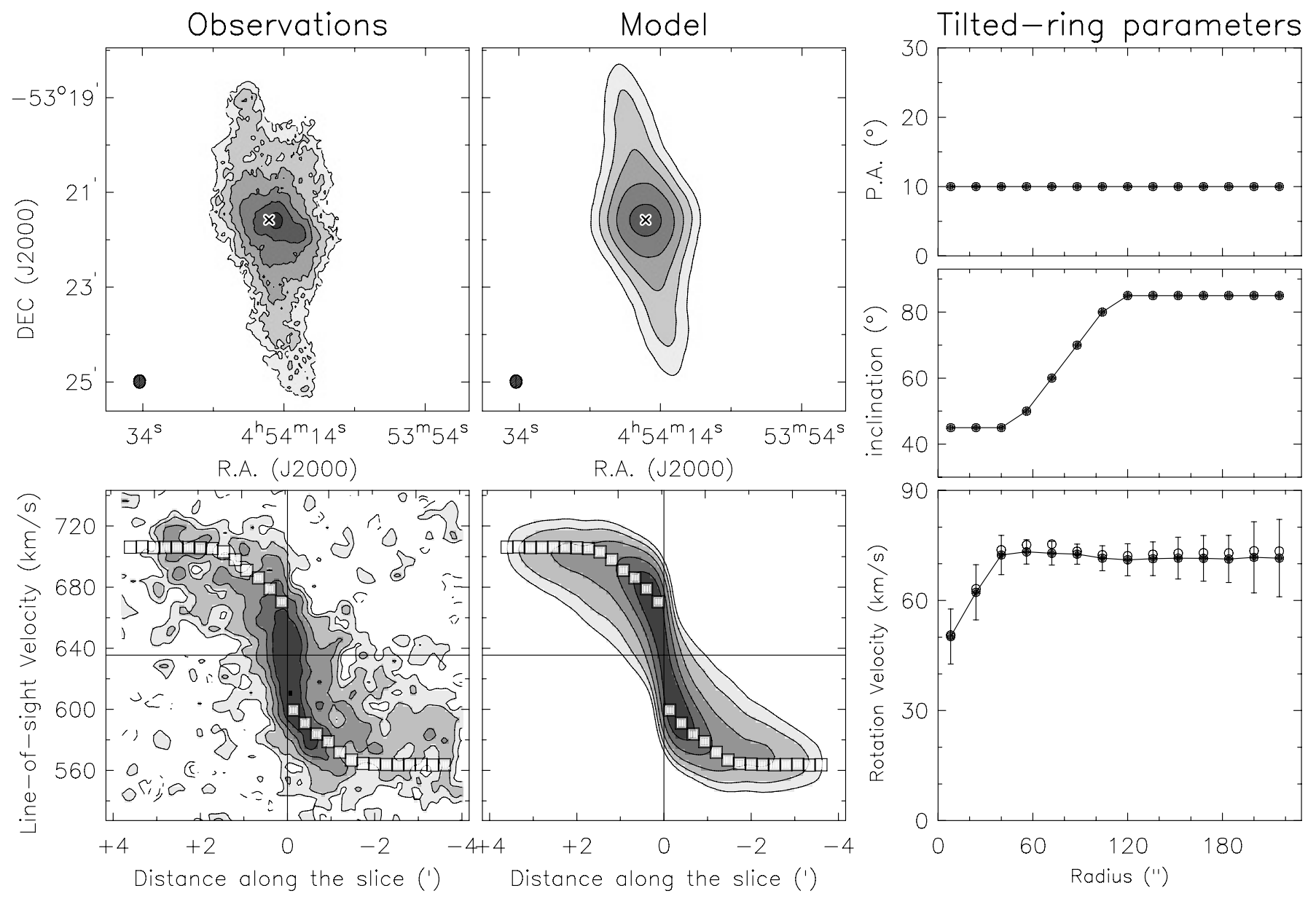

Fig. 5. The warped galaxy NGC 1705. Left: total H I map at $\sim 16^{\prime \prime}$ resolution (top) and PV-diagrams at $20^{\prime \prime}$ resolution (bottom) obtained from the observed cube and the model-cube. In the total H I map, contours are at 1.1, 2.2, 4.4, 8.8, $17.6 \times 10^{20}$ atoms $\mathrm{cm}^{-2}$; the circle to the bottom-left shows the beam. In the PV-diagrams, the contours are at -1.5 (dashed), 1.5, 3, $6,12,24 \sigma$; the squares show the rotation curve projected along the line of sight. Right: tilted-ring parameters for the disk model. The open circles show the asymmetric-drift-corrected rotation curve. See Sect. 5 for details.

and strongly warped in the outer parts. A tilted-ring fit to the VF, therefore, poses severe limitations when determining the dependence of $\mathrm{PA}_{\text {kin }}$ and $i_{\text {kin }}$ on radius (cf. Begeman 1987). We built a series of disk models assuming different types of warps. In the following, we only discuss our best models (shown in Figs. 4 and 5). For both galaxies, the warp is slightly asymmetric between the approaching and receding sides of the disk, and the actual dependence of $i_{\text {kin }}$ on radius remains uncertain.

For NGC 4214, we found that $\mathrm{PA}_{\text {kin }} \simeq 65^{\circ}$ and $i_{\text {kin }} \simeq 30^{\circ}$ for $R \lesssim 3^{\prime}$, in agreement with the optical values within the uncertainties, while at larger radii $\mathrm{PA}_{\text {kin }} \simeq 84^{\circ}$ and $i_{\text {kin }}$ gradually decreases (see Fig. 4, right panels). This model provides a good match to the observed VF and PV-diagram taken along the line of nodes (see Fig. 4, left panels); minor discrepancies $\left(\sim 20 \mathrm{~km} \mathrm{~s}^{-1}\right)$ are observed, possibly due to non-circular motions caused by the inner stellar bar and/or streaming motions along the prominent H I spiral arms (see the H I map in Appendix C).

The HI disk of NGC 1705 appears highly inclined in the outer parts, thus the VF does not provide useful information regarding the dependence of $i_{\text {kin }}$ on radius. For this galaxy, we built 3D models using an axisymmetric $\mathrm{HI}$ distribution in each ring, and determined the values of $i_{\text {kin }}$ by comparing total H I maps and PV-diagrams obtained from the observed cube and the model-cubes. Similarly to Elson et al. (2013), we found that $\mathrm{PA}_{\text {kin }} \simeq 10^{\circ}$ while $i_{\text {kin }}$ abruptly changes at $R \simeq 1.5^{\prime}$ (see
Fig. 5, right panels). We adopted, however, a higher value of $i_{\text {kin }}$ in the outer parts than Elson et al. (2013) $\left(85^{\circ}\right.$ instead of $\left.65^{\circ}\right)$. This is necessary to reproduce the tails of $\mathrm{HI}$ emission towards $V_{\text {sys }}$ that are clearly visible in PV-diagrams at $20^{\prime \prime}$ resolution (see Fig. 5, bottom panels). To fully reproduce these broad H I profiles, especially the H I emission in the "forbidden" quadrants of the PV-diagram, we also had to include radial motions of $\sim 15 \mathrm{~km} \mathrm{~s}^{-1}$ (cf. with NGC 2915, Elson et al. 2011). As a consequence of the high value of $i_{\text {kin }}$ in the outer parts, the thickness of the $\mathrm{H}$ I disk must be $\sim 500 \mathrm{pc}$ in order to reproduce the observed total H I map (see Fig. 5, top panels).

\subsection{Asymmetric-drift correction}

For several galaxies in our sample, the observed rotation velocity $V_{\text {rot }}$ is only a factor $\sim 2$ or 3 larger than the H I velocity dispersion $\sigma_{\mathrm{HI}}$. In order to trace the underlying mass distribution, the observed rotation curves have to be corrected for pressure support. We calculated the asymmetric-drift correction following Meurer et al. (1996). We assumed that i) the H I kinematics is axisymmetric, ii) the H I velocity dispersion is isotropic; iii) the velocity dispersion and the scale-height of the $\mathrm{HI}$ disk are constant with radius; and iv) the $\mathrm{HI}$ surface density profile can be approximated either by an exponential function $\Sigma_{0, \mathrm{HI}} \times \exp \left(-R / R_{0, \mathrm{HI}}\right)$ or by a Gaussian function $\Sigma_{0, \mathrm{HI}} \times \exp \left[-\left(R-R_{0, \mathrm{HI}}\right)^{2} /\left(2 s^{2}\right)\right]$. 


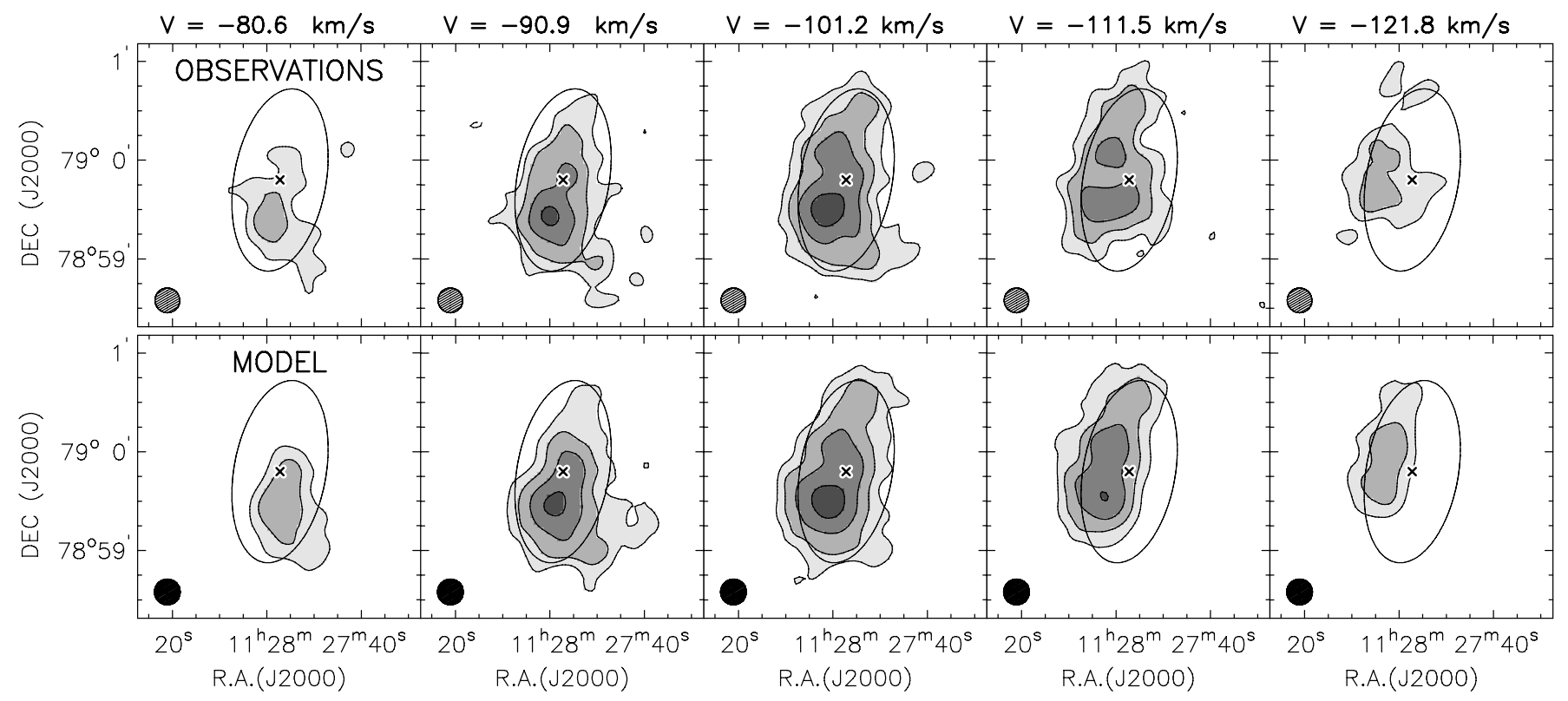

Fig. 6. Channel maps for UGC 6456 (VII Zw 403). Top: observations. Bottom: a 3D disk model with $V_{\text {rot }} \simeq V_{\text {rad }} \simeq \sigma_{\mathrm{HI}} \simeq 10 \mathrm{~km} \mathrm{~s}{ }^{-1}$. Contours are at $3,6,12,24 \sigma$. The cross shows the optical center. The ellipse corresponds to the $R$-band isophote at $25 \mathrm{mag} \operatorname{arcsec}^{-2}$.

The circular velocity $V_{\text {circ }}$, corrected for asymmetric-drift, is thus given by

$V_{\text {circ }}=\sqrt{V_{\text {rot }}^{2}+\sigma_{\mathrm{HI}}^{2}\left(R / R_{0, \mathrm{HI}}\right)}$

in the case of an exponential surface density profile, and by

$V_{\text {circ }}=\sqrt{V_{\text {rot }}^{2}+\sigma_{\mathrm{HI}}^{2} R\left(R-R_{0, \mathrm{HI}}\right) / s^{2}}$

in the case of a Gaussian surface density profile. For NGC 1705, the first assumption is not valid because the H I disk is strongly warped with an abrupt change of the inclination by $\sim 40^{\circ}$ (see Fig. 5), thus we calculated the asymmetric-drift correction separately for the inner, face-on disk and for the outer, edge-on disk.

Table 4 provides the values of $\sigma_{\mathrm{HI}}$, derived by building 3D disk models, and the parameters for the exponential/Gaussian functions, obtained by fitting the observed H I surface density profiles. For NGC 1705, we fitted the H I surface density profile with 2 different exponential functions for the inner face-on disk and the outer edge-on disk. The open-circles in Figs. 3-5 (right panels) show the asymmetric-drift-corrected rotation curves. The correction is generally smaller than the error bars, except for SBS $1415+437$ that is rotating at only $\sim 20 \mathrm{~km} \mathrm{~s}^{-1}$ (see also UGC 4483 in Lelli et al. 2012b).

\subsection{Non-circular motions}

The PV-diagrams in Figs. 3-5 clearly show that the HI kinematics of these galaxies is dominated by rotation. In several cases, however, a simple rotating-disk model cannot reproduce all the features of the observed cube. In Lelli et al. (2012a,b), we showed that the H I disks of I Zw 18 and UGC 4483 likely have radial motions of $\sim 15$ and $\sim 5 \mathrm{~km} \mathrm{~s}^{-1}$, respectively. We did not find such regular radial motions in the 7 BCDs analyzed here, with the possible exception of NGC 1705 that shows double-peaked H I profiles near the center and may have radial motions of $\sim 15 \mathrm{~km} \mathrm{~s}^{-1}$. Several galaxies, however, do show kinematically-anomalous components that deviate from the main rotation pattern of the disk. We briefly discuss 3 interesting cases: NGC 1705, NGC 2366, and NGC 4214.

NGC 1705 has a H I "spur" to the North-West (see Meurer et al. 1998; Elson et al. 2013), that may be interpreted as an $\mathrm{HI}$ outflow associated with the $\mathrm{H} \alpha$ wind (Meurer et al. 1992). The VF of NGC 2366 shows a strong distortion to the North-West (see Appendix C), that Oh et al. (2008) interpreted as non-circular motions of $\sim 20 \mathrm{~km} \mathrm{~s}^{-1}$ (see their Fig. 3). Finally, NGC 4214 shows non-circular motions of $\sim 20$ to $\sim 50 \mathrm{~km} \mathrm{~s}^{-1}$ (corrected for $i$ ) that are visible in several PV-diagrams taken across the disk (not shown here, but some kinematically-anomalous gas can be seen in the bottom panels of Fig. 4); these non-circular motions are likely associated with the H I spiral arms and/or the inner stellar bar.

\section{Galaxies with a kinematically disturbed H I disk}

Seven galaxies in our sample have a kinematically disturbed HI disk: NGC 625, NGC 1569, NGC 4163, NGC 4449, NGC 5253, UGC 6456, and UGC 9128. For these objects, we used channels maps and PV-diagrams to estimate $\left(\alpha_{\text {kin }}, \delta_{\text {kin }}\right)$, $V_{\text {sys }}, \mathrm{PA}_{\text {kin }}$, and the rotation velocity in the outer parts. For the inclination, we assumed the optical value. The values of the kinematic parameters have been tested by building 3D disk models with constant $V_{\text {rot }}$. These models cannot reproduce the observations in details, but indicate that the observed H I kinematics are consistent with a rotating disk. The resulting values for the kinematic parameters are rather uncertain.

Two galaxies (NGC 5253 and UGC 6456) show a regular velocity gradient approximately along the HI minor axis (see the VFs in Appendix C). Velocity gradients along the H I minor axis have also been observed in other BCDs and interpreted as unusual rotation around the major axis (e.g., Thuan et al. 2004; Bravo-Alfaro et al. 2004). This peculiar kinematic behavior, however, can also be interpeted as a H I disk with strong radial motions (e.g., López-Sánchez et al. 2012). We built 3D disk models assuming the PA suggested by the total H I map (consistent with the optical value within the errors) and tried different combinations of circular and radial motions. Our best-models are shown in Figs. 6 and 7. For UGC 6456, the 


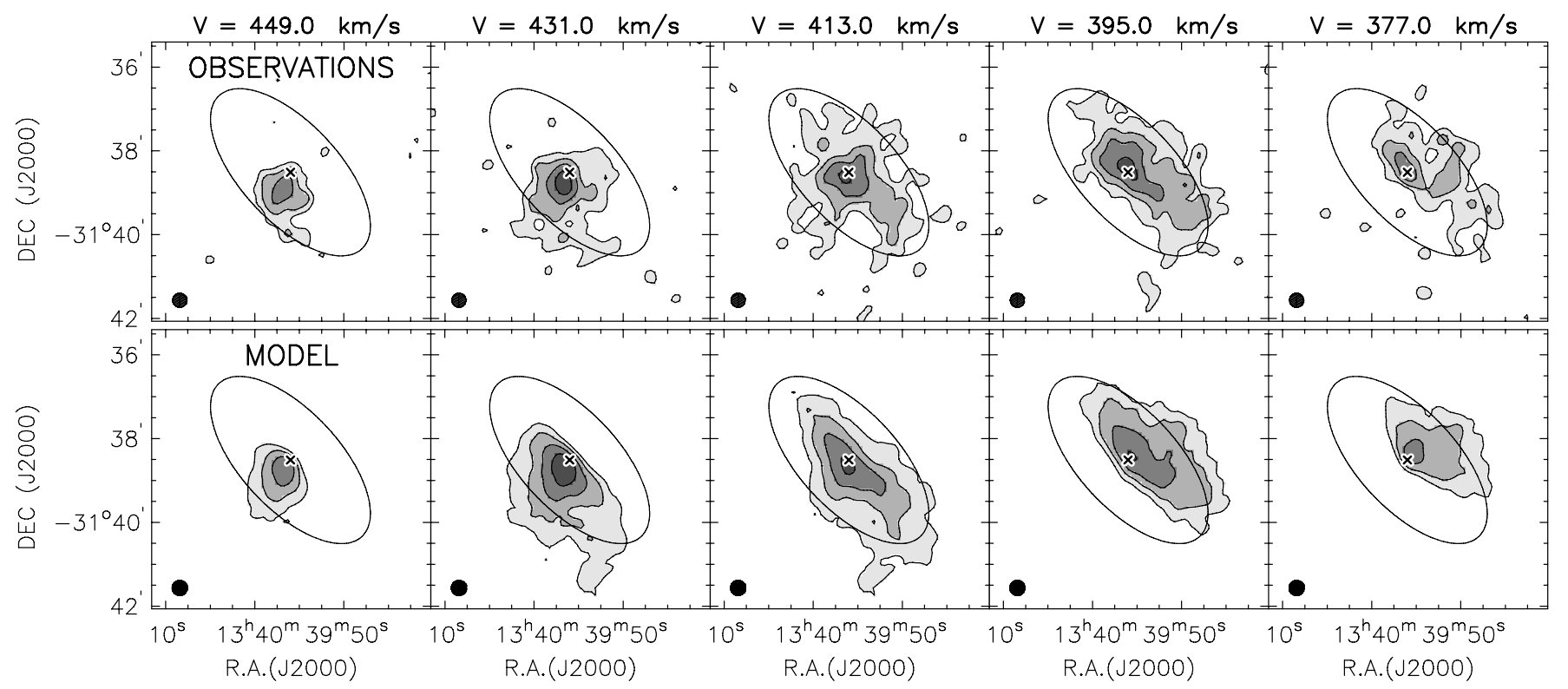

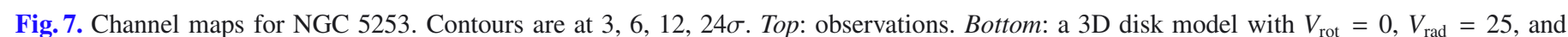
$\sigma_{\mathrm{HI}}=15 \mathrm{~km} \mathrm{~s}^{-1}$. The cross shows the optical center. The ellipse corresponds to the $R$-band isophote at $24 \mathrm{mag}_{\operatorname{arcsec}}{ }^{-2}$.

H I emission can be reproduced by a combination of circular and radial motions ( $V_{\text {rot }} \simeq V_{\text {rad }} \simeq 10 \mathrm{~km} \mathrm{~s}^{-1}$, see Fig. 6). The case of NGC 5253 is even more extreme, as the radial component is $\sim 25 \mathrm{~km} \mathrm{~s}^{-1}$ while the rotation is constrained to be $\$ 5 \mathrm{~km} \mathrm{~s}^{-1}$ (see Fig. 7). In our opinion, strong radial motions are a more likely interpretation than rotation around the major axis, since gas inflows/outflows are expected in starburst galaxies.

For UGC 6456, it is not possible to distinguish between inflow and outflow, as it is unknown which side of the disk is nearest to the observer. For NGC 5253, instead, shadowing of the X-ray emission suggests that the southern side of the disk is the nearest one (Ott et al. 2005a), implying that the radial motion is an inflow. For both galaxies, the inflow timescale $t_{\text {in }}=R_{\mathrm{HI}} / V_{\text {rad }}$ is $\sim 100-200 \mathrm{Myr}$, thus comparable with the starburst duration (see McQuinn et al. 2010b). We also calculated the gas inflow rates $\dot{M}_{\text {in }}=1.33 M_{\mathrm{HI}} / t_{\text {in }}$ (the factor 1.33 takes the contribution of helium into account) and found that they are about 1 order of magnitude higher than the current SFRs (from McQuinn et al. 2010a): for NGC $5253 \dot{M}_{\text {in }} \simeq$ $1.5 M_{\odot} \mathrm{yr}^{-1}$ and $S F R \simeq 0.16 M_{\odot} \mathrm{yr}^{-1}$, while for UGC 6456 $\dot{M}_{\text {in }} \simeq 0.3 M_{\odot} \mathrm{yr}^{-1}$ and $S F R \simeq 0.02 M_{\odot} \mathrm{yr}^{-1}$. Similar results can be derived for I Zw 18 and UGC 4483, which show a global radial motion superimposed on a regularly rotating $\mathrm{HI}$ disk (Lelli et al. 2012a,b): for I Zw $18 \dot{M}_{\text {in }} \simeq 1 M_{\odot} \mathrm{yr}^{-1}$ and $S F R \simeq 0.1 M_{\odot} \mathrm{yr}^{-1}$, while for UGC $4483 \dot{M}_{\mathrm{in}} \simeq 0.1 M_{\odot} \mathrm{yr}^{-1}$ and $S F R \simeq 0.01 M_{\odot} \mathrm{yr}^{-1}$. If the hypothesis of a radial inflow is correct, these results would imply that only $\sim 10 \%$ of the inflowing gas is converted into stars, in line with several estimates of the star-formation efficiencies in dwarf galaxies (e.g., Leroy et al. 2008).

\section{Mass models}

\subsection{Preliminary considerations}

In Sect. 5 we derived rotation curves for nine BCDs with a regularly rotating $\mathrm{HI}$ disk, while in Sect. 6 we estimated the outer rotation velocities of seven BCDs with a kinematically disturbed HI disk. In several cases, we found that the values for the kinematical center, PA, and $i$ do not coincide with the optical ones (see Table B.2). Non-circular motions are also present in the HI disks of several galaxies. This raises the question as to whether the $\mathrm{HI}$ disks are in a fully-stable configuration and the observed rotation velocities are suitable to investigate the mass distributions in these galaxies.

In Fig. 8, we consider the location of the starbursting dwarfs in our sample on the baryonic Tully-Fisher relation (BTFR, McGaugh et al. 2000). We exclude UGC 6456 and NGC 5253 as their H I kinematics seems to be dominated by radial motions (Figs. 6 and 7), and UGCA 290 and UGC 6541 as they have unsettled H I distributions. We use data from McGaugh (2005) for star-dominated galaxies (grey crosses) and McGaugh (2012) for gas-dominated ones (grey dots). Most BCDs with a regularly rotating $\mathrm{H}$ I disk have flat rotation curves in the outer parts (Figs. 3-5), thus we use the mean value of $V_{\text {circ }}$ along the flat part (Verheijen 2001). In two cases (NGC 4068 and NGC 6789), however, the rotation curve does not reach the flat part, thus we use the maximum observed value of $V_{\text {circ }}$. One would expect these galaxies to deviate from the BTFR if $V_{\text {circ }}$ keeps rising after the last measured point. For BCDs with a kinematically disturbed H I disk, our estimates of $V_{\text {circ }}$ are considered as lower limits in Fig. 8 because i) they may not correspond to the flat part of the rotation curve, and ii) they have not been corrected for pressure support. As in McGaugh $(2005,2012)$, the total baryonic mass is calculated as $M_{*}+1.33 M_{\mathrm{HI}}$; the possible contributions of molecular and ionized gas are neglected. Note that McGaugh $(2005,2012)$ estimated $M_{*}$ using integrated colors and synthetic stellar population models, while our estimate of $M_{*}$ is based on the HST studies of the resolved stellar populations.

Figure 8 shows that both BCDs with a regularly rotating H I disk (filled symbols) and BCDs with a kinematically disturbed $\mathrm{H}$ I disk (open symbols) follow the BTFR within the uncertainties, except for NGC 4163, NGC 4449, and SBS 1415+437. Thus, for the majority of galaxies in our sample, the observed rotation velocity is a reasonable proxy for the total dynamical mass within the H I radius. The three objects that strongly deviate from the BTFR may be unusual for the following reasons. NGC 4163 has a disturbed H I distribution with tails and plumes; it is unclear whether the observed velocity gradient (of only 


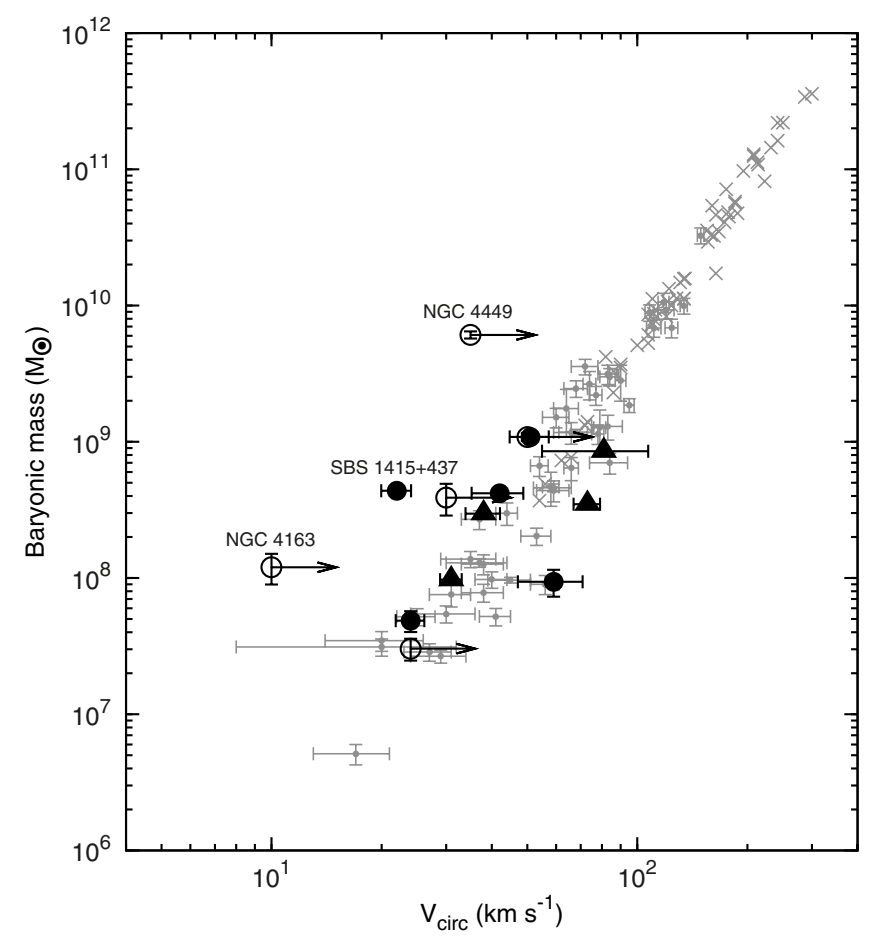

Fig. 8. Location of starbursting dwarfs on the baryonic TullyFisher relation. Grey-crosses indicate star-dominated galaxies from McGaugh (2005), while grey-dots indicate gas-dominated galaxies from McGaugh (2011). Filled and open symbols indicate, respectively, BCDs with a regularly rotating $\mathrm{HI}$ disk and BCDs with a kinematically disturbed HI disk. For the latter ones, the circular velocity may be underestimated. The triangles indicate galaxies for which the baryonic mass is a lower limit. Galaxies that significantly deviate from the relation are labeled.

$\sim 10 \mathrm{~km} \mathrm{~s}^{-1}$ ) is really due to rotation. NGC 4449 is characterized by two counter-rotating gas systems (see VF in Appendix C and Hunter et al. 1999); we estimated the rotation velocity of the inner H I disk, that possibly does not correspond to the asymptotic velocity along the flat part of the rotation curve. It is unclear whether the outer gas system forms a rotating disk; intriguingly, its inferred circular velocity would be consistent with the BTFR. Finally, SBS $1415+437$ has a kinematic center that is strongly off-set $(\sim 800 \mathrm{pc})$ with respect to the optical center and to the centroid of the HI distribution; the lopsided HI morphology and kinematics may be explained by a pattern of elliptical orbits viewed almost edge-on (cf. Baldwin et al. 1980). A detailed investigation of these models is beyond the scope of this paper, but it is clear that the observed rotation curve might not be a reliable tracer of the dynamical mass.

Considering the uncertainties involved, we proceed as follows. In Sect. 7.2 we estimate global baryonic fractions within $R_{\text {opt }}$ for the 14 objects in Fig. 8, while in Sect. 7.3 we build detailed mass models for four galaxies with a regularly rotating disk (NGC 2366, NGC 6789, NGC 4068, and UGC 4483). In Lelli et al. (2012a,b), we presented similar mass models for I Zw 18 and UGC 4483, respectively. For I Zw 18, the stellar mass from the HST studies is very uncertain, thus we built mass models assuming a maximum-disk. For UGC 4483, instead, we used the HST information on the stellar mass to break the "diskhalo degeneracy" (van Albada \& Sancisi 1986); here we extend our previous analysis on UGC 4483 by making different assumptions about the IMF and including the gravitational contribution of molecules. We do not decompose the rotation curves of NGC 1705, I Zw 36, and SBS $1415+437$ because the optical and kinematical centers show a strong off-set $(\gtrsim 1$ disk scale length), thus it is not possible to calculate the gravitational contributions of stars, gas, and DM using a common dynamical center. We also exclude NGC 4214 because the H I disk is close to face-on and strongly warped.

\subsection{Baryonic fractions}

As we mentioned in Sect. 2, the HST studies of the resolved stellar populations provide a direct estimate of the total stellar mass of a galaxy. For seven objects, however, these stellar masses are lower limits because either the HST field of view only covers a small portion of the galaxy (NGC 1705, NGC 4214, and NGC 625), or the ancient SFH (>1 Gyr) is uncertain due to the limited photometric depth (I Zw 18 and UGCA 290), or both (I Zw 36 and UGC 6541). Moreover, the values of $M_{*}$ depend on the assumed IMF and on the mass returned to the interstellar medium (ISM) by stellar ejecta. The stellar masses in Table 1 are calculated assuming a Salpeter IMF from 0.1 to $100 M_{\odot}$ and a gas-recycling efficiency of $30 \%$; a Kroupa IMF would give stellar masses lower by a factor of 1.6 (McQuinn et al. 2012). In Table B.3, we provide three different estimates for the baryonic mass within $R_{\text {opt }}$ (in order of increasing mass): i) $M_{\mathrm{bar}}^{\mathrm{Kr}}=M_{*}^{\mathrm{Kr}}+1.33 M_{\mathrm{HI}}\left(R_{\mathrm{opt}}\right)$, where $M_{*}^{\mathrm{Kr}}$ is the stellar mass assuming a Kroupa IMF and $M_{\mathrm{HI}}\left(R_{\mathrm{opt}}\right)$ is the HI mass within $R_{\mathrm{opt}}$; ii) $M_{\mathrm{bar}}^{\mathrm{Sal}}=M_{*}^{\mathrm{Sal}}+1.33 M_{\mathrm{HI}}\left(R_{\mathrm{opt}}\right)$, where $M_{*}^{\text {Sal }}$ is the stellar mass assuming a Salpeter IMF; and iii) $M_{\mathrm{bar}}^{\mathrm{mol}}=M_{*}^{\text {Sal }}+1.33 M_{\mathrm{HI}}\left(R_{\mathrm{opt}}\right)+M_{\mathrm{mol}}$, where we also include an indirect estimate of the molecular mass $M_{\text {mol }}$.

The molecular content of dwarf galaxies is very uncertain as they usually have low metallicities and the CO-line, which traces the molecular hydrogen, is often undetected (e.g., Taylor et al. 1998). Moreover, even when the CO-line is detected, the conversion factor from $\mathrm{CO}$ luminosity to $\mathrm{H}_{2}$ mass is poorly constrained, as it may differ from the Milky-Way value and vary with metallicity and/or other physical conditions (e.g., Boselli et al. 2002). Thus, we chose to indirectly estimate the molecular mass by using the correlation between SFR surface density $\Sigma_{\text {SFR }}$ and $\mathrm{H}_{2}$ surface density $\Sigma_{\mathrm{H}_{2}}$ (e.g., Bigiel et al. 2008). In particular, we use Eq. (28) of Leroy et al. (2008), which assumes that the star-formation efficiency in dwarf galaxies is the same as in spirals (but see also Roychowdhury et al. 2011). This equation can be written as:

$$
M_{\mathrm{H}_{2}}\left[M_{\odot}\right]=1.9 \times 10^{9} \operatorname{SFR}\left[M_{\odot} \mathrm{yr}^{-1}\right]
$$

We use the average SFR over the last $\sim 10$ Myr as obtained by the HST studies, and assume a systematic uncertainty of $30 \%$ on $M_{\mathrm{H}_{2}}$. The real uncertainty, however, may be larger as starbursting dwarfs may deviate from the $\Sigma_{\mathrm{H}_{2}}-\Sigma_{\mathrm{SFR}}$ relation.

We used the three different estimates of the total baryonic mass to calculate baryonic fractions $f_{\mathrm{bar}}=M_{\mathrm{bar}} / M_{\mathrm{dyn}}$ within $R_{\text {opt }}$. Assuming a spherical mass distribution, the dynamical mass within $R_{\text {opt }}$ is given by $M_{\text {dyn }}\left(R_{\text {opt }}\right)=V_{\text {circ }}^{2}\left(R_{\text {opt }}\right) \times R_{\text {opt }} / G$, where $V_{\text {circ }}\left(R_{\text {opt }}\right)$ is the asymmetric-drift-corrected circular velocity at $R_{\text {opt }}$. We estimated the errors on $M_{\text {dyn }}\left(R_{\text {opt }}\right)$ considering i) the random errors $\delta_{V_{\text {circ }}}$ on $V_{\text {circ }}$ due to possible kinematic asymmetries and/or non-circular motions, and ii) the errors $\delta_{i}$ on the inclination angle, which would cause a systematic shift of all the velocity-points but preserve the overall shape of the rotation curve (unless the $\mathrm{HI}$ disk is warped). Considering that 


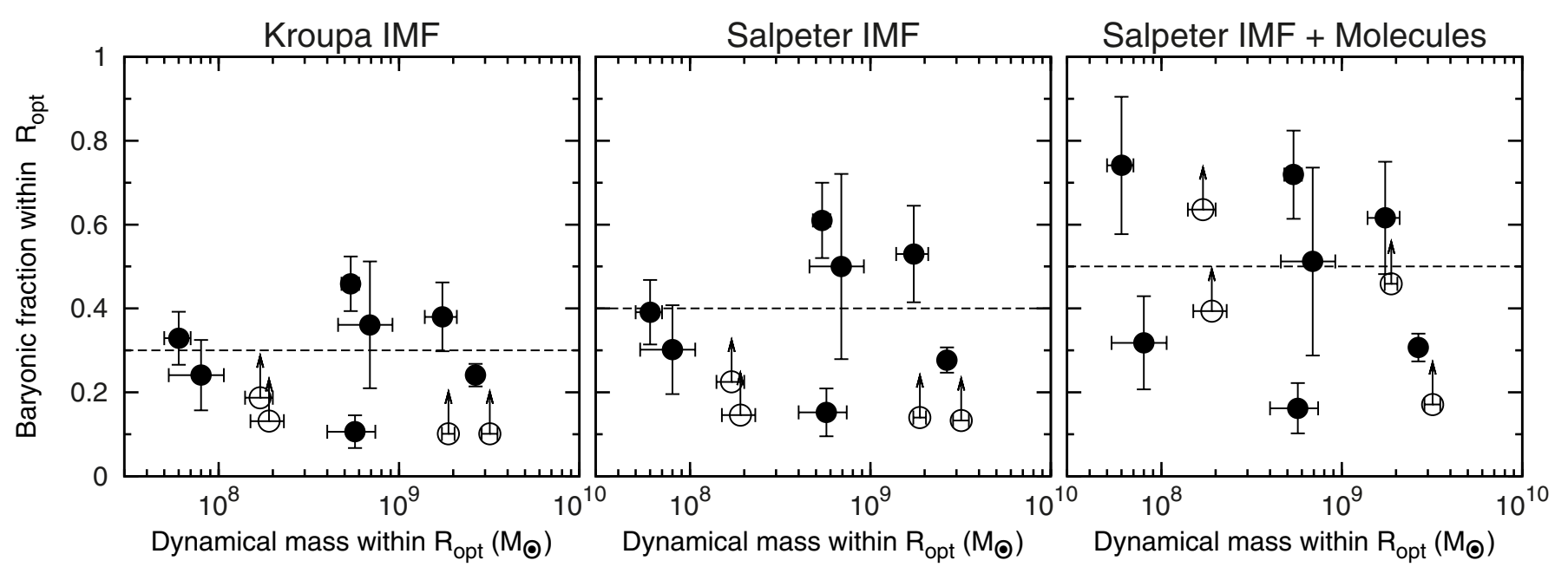

Fig. 9. Baryonic fractions $f_{\mathrm{bar}}=M_{\mathrm{bar}} / M_{\mathrm{dyn}}$ versus $M_{\mathrm{dyn}}$ calculated within the optical radius $R_{\mathrm{opt}}$. The open circles indicate lower limits for $f_{\mathrm{bar}}$. The dashed lines indicate the average value of $f_{\text {bar }}$ for the different assumptions on $M_{\text {bar }}$. See Sect. 7.2 for details.

Table 5. Results of the rotation curve decompositions.

\begin{tabular}{|c|c|c|c|c|c|c|c|c|c|}
\hline \multirow[t]{2}{*}{ Galaxy } & \multicolumn{3}{|c|}{$F_{\text {bar }}\left(2.2 R_{\mathrm{d}}\right)$} & \multicolumn{3}{|c|}{$\begin{array}{c}\rho_{0} \\
\left(10^{-3} M_{\odot} \mathrm{pc}^{-3}\right)\end{array}$} & \multicolumn{3}{|c|}{$\begin{array}{c}r_{\mathrm{c}} \\
(\mathrm{kpc})\end{array}$} \\
\hline & Kroupa & Sal. & Sal.+ Mol. & Kroupa & Sal. & Sal.+ Mol. & Kroupa & Sal. & Sal.+ Mol. \\
\hline NGC 2366 & 0.5 & 0.6 & 0.6 & 37 & 34 & 31 & 1.2 & 1.3 & 1.3 \\
\hline NGC 4068 & 0.9 & 1.2 & 1.3 & 14 & 7 & 3 & 1.8 & 1.8 & 1.8 \\
\hline NGC 6789 & 0.4 & 0.5 & 0.5 & 450 & 397 & 384 & 0.7 & 0.7 & 0.7 \\
\hline UGC 4483 & 0.6 & 0.7 & 0.9 & 122 & 87 & 15 & 0.3 & 0.4 & 2.0 \\
\hline
\end{tabular}

Notes. For NGC 4068 and NGC 6789, the values of $r_{\mathrm{c}}$ are unconstrained, thus we assumed $r_{\mathrm{c}}=R_{\mathrm{opt}}$ (in italics).

$V_{\text {circ }} \simeq V_{\text {los }} / \sin (i)$, the error on $M_{\text {dyn }}$ is thus given by

$\delta_{M_{\mathrm{dyn}}}=\left\{\frac{2 V_{\mathrm{circ}} R}{G} \sqrt{\delta_{V_{\text {circ }}}^{2}+\left[\frac{V_{\mathrm{circ}} \delta_{i}}{\tan (i)}\right]^{2}}\right\}_{R=R_{\mathrm{opt}}}$

The resulting dynamical masses and baryonic fractions are listed in Table B.3. The errors on $f_{\mathrm{bar}}$ have been calculated by propagating the various uncertainties on $M_{*}, M_{\mathrm{gas}}\left(R_{\mathrm{opt}}\right), M_{\mathrm{mol}}$, and $M_{\text {dyn }}\left(R_{\text {opt }}\right)$. The three galaxies that deviate from the BTFR (NGC 4163, NGC 4449, and SBS 1415+437) have unphysically large baryonic fractions $\left(f_{\text {bar }} \gtrsim 1\right)$, further suggesting that the observed circular velocities are not adequate to trace the dynamical mass. For the remaining 11 objects, we plot $f_{\text {bar }}$ against $M_{\text {dyn }}$ in Fig. 9. We find no clear trend with $M_{\text {dyn }}$ or with other physical parameters such as $M_{*}, M_{\mathrm{HI}}, \mathrm{SFR}_{\mathrm{p}}$, and $t_{\mathrm{p}}$ (see Table 1). The mean baryonic fractions within the stellar component are relatively high: $\sim 0.3$ for a Kroupa IMF, $\sim 0.4$ for a Salpeter IMF, and $\sim 0.5$ for a Salpeter IMF plus molecules. Old stars (with ages $>1$ Gyr) generally provide the major contribution to these baryonic fractions, except for a few cases where either the atomic gas dominates (NGC 2366) or the molecular gas may be very important (NGC 1705 and UGC 4483, see Table B.3).

\subsection{Rotation curve decompositions}

In this section, we decompose the rotation curves of four galaxies (NGC 2366, NGC 4068, NGC 6789, and UGC 4483), which have a regularly rotating $\mathrm{HI}$ disk centered on the stellar component. We follow standard procedures described by
Begeman (1987). Similarly to Sect. 7.2, we compute three different mass models which assume i) a Kroupa IMF; ii) a Salpeter IMF; and iii) a Salpeter IMF plus the molecular mass inferred by Eq. (4). These mass models are shown in Fig. 10.

The gravitational contribution of the atomic gas was calculated using the H I surface density profiles and scaled to the total atomic gas mass as $\sqrt{1.33 M_{\mathrm{HI}}}$. In agreement with the models in Sect. 5.1, we assume that the gaseous disk has an exponential vertical density distribution with a scale height of $100 \mathrm{pc}$.

The gravitational contribution of the stellar component $V_{*}(R)$ was calculated using $R$-band surface brightness profiles and assuming that the stars are located in a disk with a vertical density distribution given by $\operatorname{sech}^{2}(z / z 0)$ (van der Kruit \& Searle 1981) with $z_{0}=0.3 R_{\mathrm{d}}$. If one assumes that $z_{0} / R_{\mathrm{d}}=0.2$ or $0.4, V_{*}(R)$ would change by a few percent in the inner parts $\left(R \lesssim R_{\text {opt }}\right)$. The amplitude of $V_{*}$ was scaled to the total stellar mass as $\sqrt{M_{*}}$ (assuming either a Kroupa or a Salpeter IMF). This is equivalent to using a stellar mass-to-light ratio that is constant with radius (see Table 1).

To include the possible contribution of molecular gas, we simply assume that molecules are distributed in the same way as the stars and thus scale the amplitude of $V_{*}$ by $\sqrt{M_{*}^{\text {Sal }}+M_{\text {mol }}}$. We also tried to estimate the shape of the molecular gas contribution $V_{\text {mol }}$ using $\mathrm{H} \alpha$ and $24 \mu \mathrm{m}$ images (from Dale et al. 2009 and Gil de Paz et al. 2003). These images show very clumpy and asymmetric distributions, that are completely dominated by strong star-forming regions and shell-like structures. We thus prefer to include the molecular gas contribution in $V_{*}(R)$. We note that $B$-band and UV images may be better tracers of the 
Kroupa IMF
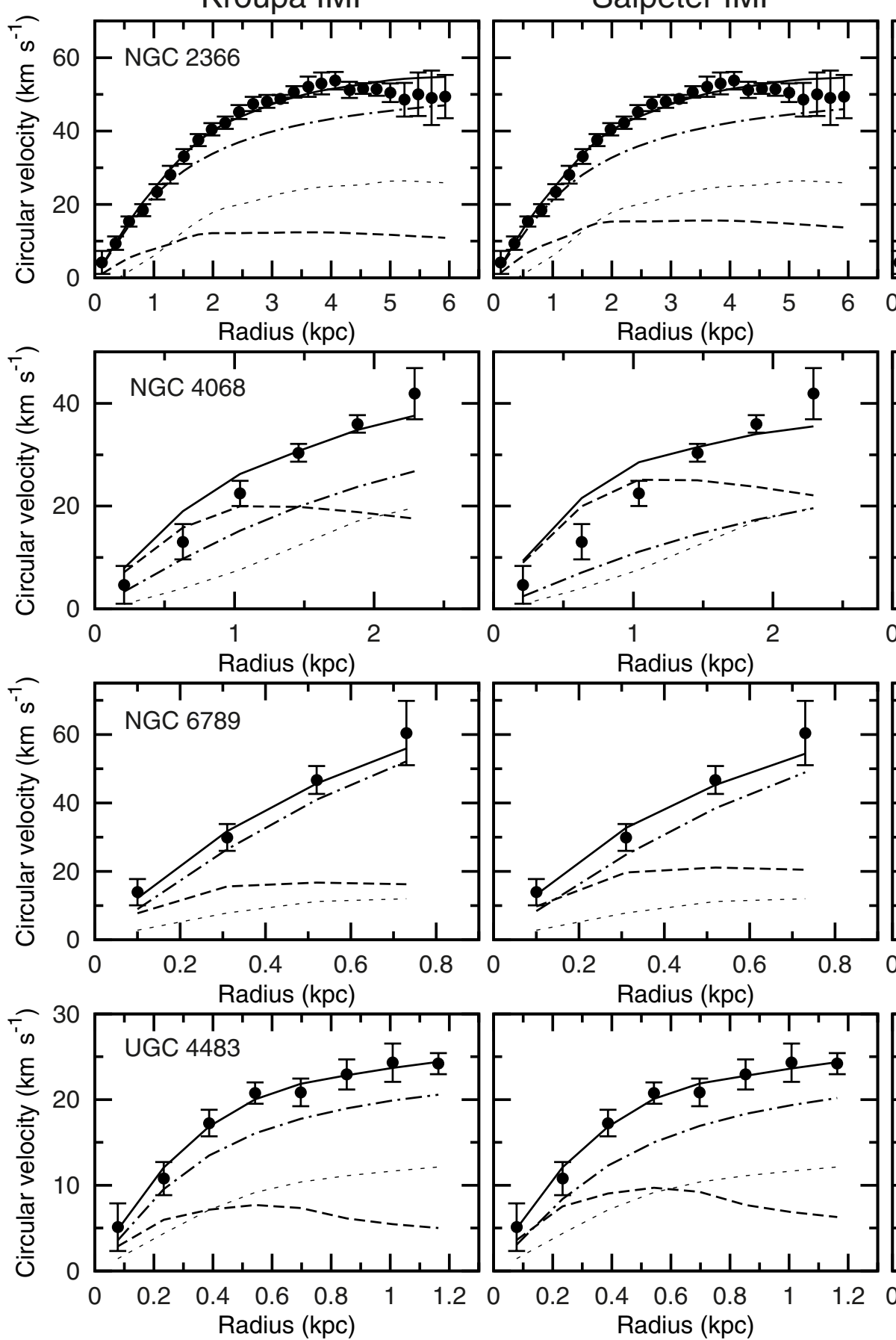

Salpeter IMF + Molecules
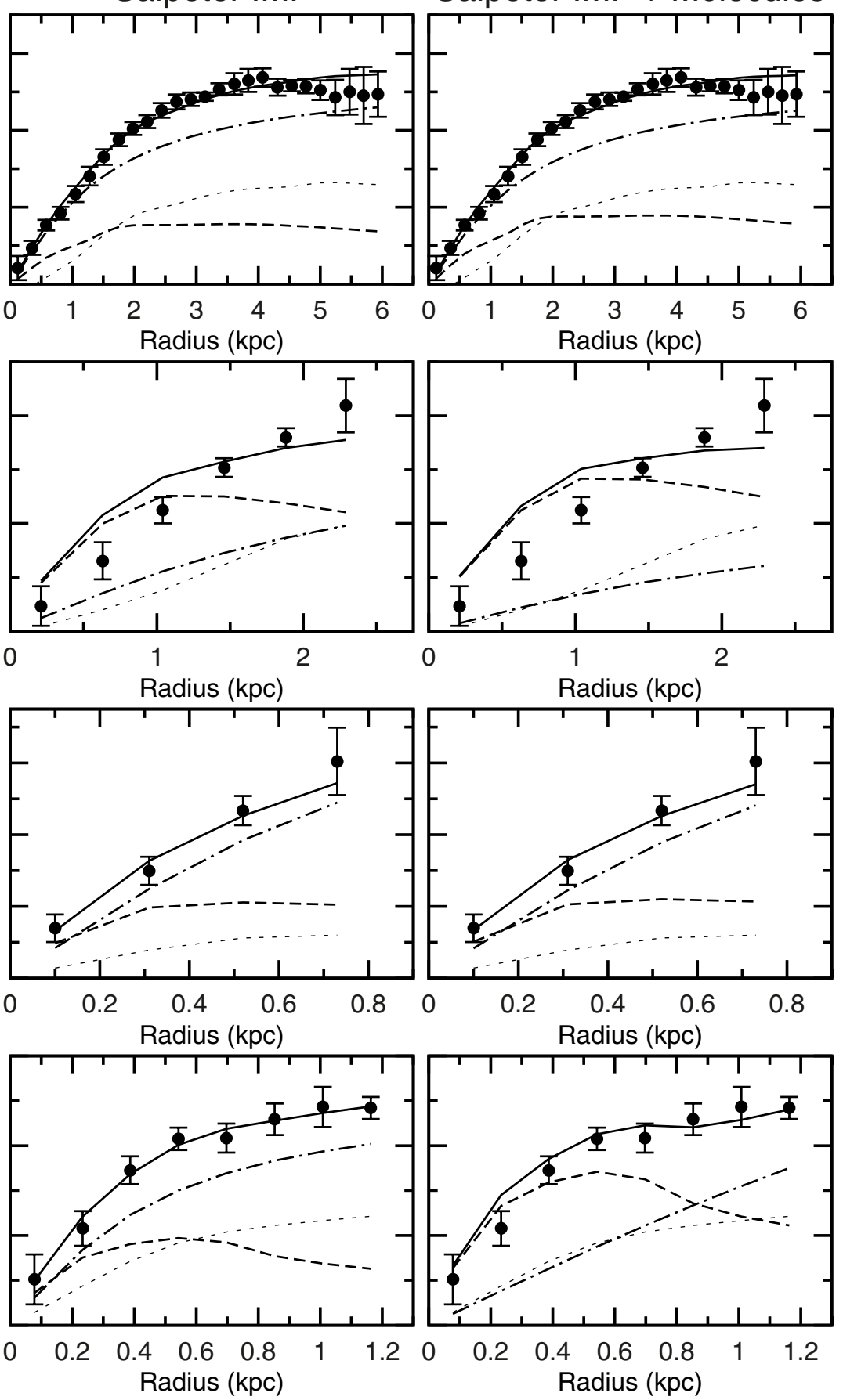

Fig. 10. Rotation curve decompositions. Dots show the observed rotation curve (corrected for asymmetric-drift). Long-dashed, short-dashed, and dot-dashed lines show the gravitational contribution due to stars, atomic gas, and dark matter, while the solid line shows the resulting total contribution to the rotation curve. See Sect. 7.3 for details.

molecular gas distribution than the $R$-band images used here. However, since the $B-R$ color profiles of BCDs typically show a sharp gradient at small radii and a flat part in the outer regions (e.g., Papaderos et al. 2002; Gil de Paz \& Madore 2005), the use of $B$-band or UV surface brightness profiles would lead to appreciable differences in $V_{*}(R)$ only at the innermost radii and have no significant effects on our general results.

For the DM distribution, we assume a pseudo-isothermal halo described by equation

$\rho_{\mathrm{ISO}}(r)=\frac{\rho_{0}}{1+\left(r / r_{\mathrm{c}}\right)^{2}}$, where the central density $\rho_{0}$ and the core radius $r_{\mathrm{c}}$ are the free parameters of the mass model. This is motivated by the fact that observed rotation curves of low-mass galaxies are well-reproduced by cored DM density profiles (e.g., Gentile et al. 2005; Oh et al. $2011 b$ ). Since the HI rotation curves are not well sampled (except for NGC 2366), the parameters of the DM halo cannot be determined with high accuracy. In particular, for NGC 6789 and NGC 4068, the core radius is completely unconstrained, thus we assumed $r_{\mathrm{c}}=R_{\mathrm{opt}}$. Given these uncertainties, we did not explore other DM density profiles than the pseudo-isothermal one. 
A baryonic disk is usually defined to be maximum if $F_{\mathrm{bar}}=$ $V_{\text {bar }} / V_{\text {circ }}=0.85 \pm 0.10$ at 2.2 disk scale lengths (Sackett 1997 ; Bershady et al. 2011), where $V_{\text {bar }}$ is the contribution to the rotation curve given by the baryons. The BCDs in Fig. 10 have $F_{\text {bar }} \simeq 0.4$ to 0.6 , except for NGC 4068 that has $F_{\text {bar }} \simeq 0.9$ for a Kroupa IMF and $F_{\text {bar }}>1$ for a Salpeter IMF (see Table 5). The latter result may suggest that a Salpeter IMF implies an unphysical, over-maximal disk for NGC 4068; however, if one assumes a nearly spherical stellar body with a scale-height of $\sim 600 \mathrm{pc}$, a Salpeter IMF would give acceptable results with $F_{\text {bar }} \lesssim 1$. The sub-maximal disks of NGC 2366, NGC 6789, and UGC 4483 are in line with the results of the DiskMass survey (e.g., Bershady et al. 2011; Westfall et al. 2011; Martinsson 2011), who measured the stellar velocity dispersions of a sample of spiral galaxies and found that $F_{\text {bar }} \simeq 0.5$. Note, however, that baryons are still dynamically significant, as $F_{\text {bar }} \simeq 0.4$ to 0.6 correspond to baryonic fractions $f_{\mathrm{bar}}=M_{\mathrm{bar}} / M_{\mathrm{dyn}} \simeq 0.2$ to 0.4 within $2.2 R_{\mathrm{d}}$, in line with the results in Sect. 7.2.

\section{Discussion}

\subsection{Comparison with other dwarf galaxies}

In Sect. 4, we compared the H I distribution and kinematics of BCDs and Irrs. In agreement with previous studies (e.g., van Zee et al. 1998, 2001), we found that BCDs have central H I densities a factor of $\sim 2$ higher than typical Irrs. The average extent of the $\mathrm{HI}$ disk with respect to the stellar component, instead, is similar for BCDs, Irrs, and spirals $\left(R_{\mathrm{HI}} / R_{\mathrm{opt}} \simeq 1.7\right.$ with $R_{\mathrm{opt}}$ defined as 3.2 exponential scale lengths $\left.R_{\mathrm{d}}\right)$. We also found that complex HI kinematics are much more common in BCDs $(\sim 50 \%)$ than in typical Irrs $(\sim 10 \%)$, likely due to the effects of stellar feedback and/or of the triggering mechanism (interactions/mergers or disk instabilities). In Lelli et al. (2014), we present a comparison between the rotation curves of BCDs and those of Irrs, and discuss the link between the starburst, the gas concentration, and the gravitational potential (see also Lelli et al. 2012a,b). Here we compare the baryonic fractions of BCDs with those of gas-rich Irrs and gas-poor spheroidals (Sphs).

For gas-rich Irrs, maximum-disk decompositions of H I rotation curves usually result in high values of the stellar mass-tolight ratio, up to $\sim 15$ in the $R$-band (e.g., Swaters et al. 2011). These high values of $M_{*} / L_{R}$ are difficult to explain using stellar population synthesis models (e.g., Zibetti et al. 2009), suggesting that Irrs are dominated by DM at all radii. The detailed baryonic fractions of Irrs, however, remain uncertain because they depend on the assumed value of $M_{*} / L_{R}$. For the BCDs in our sample, instead, we can directly calculate the values of $M_{*} / L_{R}$ using the stellar masses from the HST studies of the resolved stellar populations. Assuming a Salpeter IMF from 0.1 to $100 M_{\odot}$ and a gas-recycling efficiency of $30 \%$, we find that the mean value of $M_{*} / L_{\mathrm{R}}$ is $\sim 1.5$ (see Table 1 ). Photometric studies of BCDs (e.g., Papaderos et al. 1996) suggest that the starburst typically increases the total luminosity by a factor of $\sim 2$, whereas studies of the SFHs (e.g., McQuinn et al. 2010b) indicate that the burst only produces a small fraction of the total stellar mass $(\sim 10 \%)$. Thus, it is reasonable to assume that Irrs have, on average, $M_{*} / L_{R} \simeq 3$ for a Salpeter IMF and $M_{*} / L_{R} \simeq 2$ for a Kroupa IMF.

Similarly to Sect. 7.2, we calculated the baryonic fractions of 30 gas-rich dwarfs using the $R$-band luminosities, H I surface density profiles, and $\mathrm{HI}$ rotation curves from Swaters et al. (2002, 2009), and assuming $M_{*} / L_{R}=2$. We only considered galaxies with $i>30^{\circ}$ and high-quality rotation curves $(q>2$,

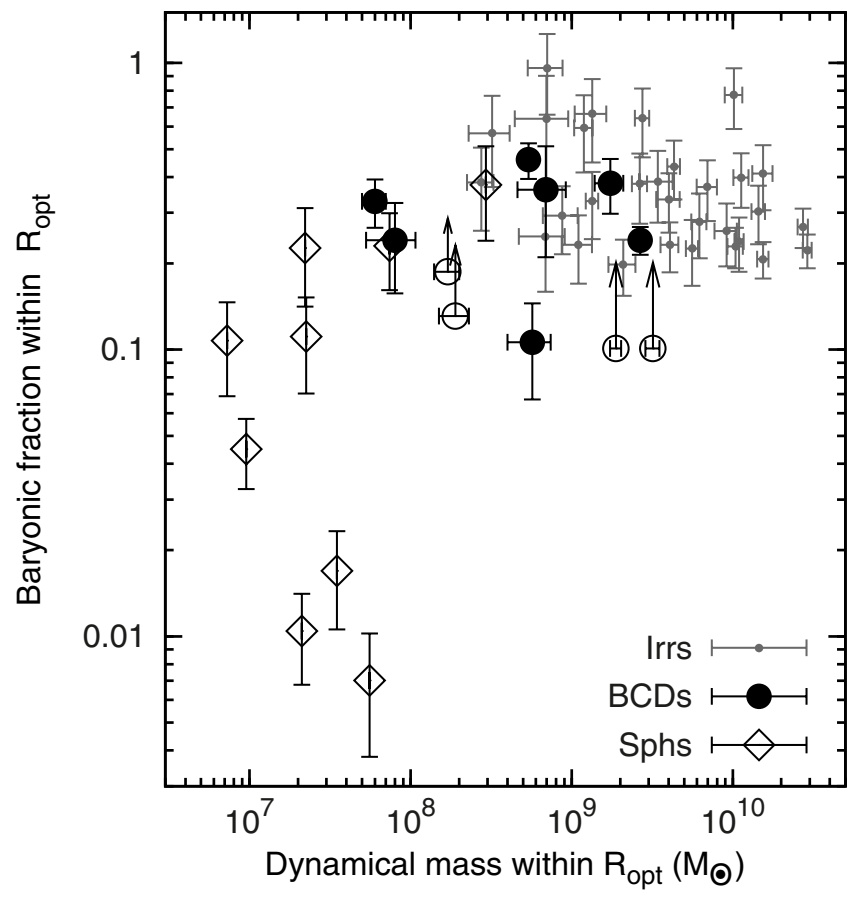

Fig. 11. Comparison between the baryonic fractions of BCDs (open and filled circles, same as Fig. 9, left panel), Irrs (grey dots), and Sphs (open diamonds). The data for Irrs and Sphs are taken from Swaters et al. (2009) and Wolf et al. (2010), respectively.

see Swaters et al. 2009), that are traced out to $\sim 3 R_{\mathrm{d}}$ and have $V_{\text {rot }}<100 \mathrm{~km} \mathrm{~s}^{-1}$ at the last measured point. We also calculated the baryonic fractions of several gas-poor dwarfs using the $V$-band luminosities and dynamical masses from Wolf et al. (2010), and assuming $M_{*} / L_{V}=2.0$. For the nearby Sphs Sculptor and Fornax, the stellar masses from de Boer et al. (2012a,b) imply, respectively, $M_{*} / L_{V} \simeq 2.2$ and $M_{*} / L_{V} \simeq 1.7$ (assuming a Kroupa IMF and a gas-recycling efficiency of $30 \%$ ). We only considered nine Sphs that have accurate estimates of the stellar velocity dispersion: the eight "classical" satellites of the Milky Way (Carina, Draco, Fornax, Leo I, Leo II, Sculptor, Sextans, and Ursa Minor) and NGC 185, which is a satellite of M31. For these Sphs, the baryonic fractions are computed at the $3 \mathrm{D}$ deprojected half-light radius $r_{1 / 2}$ (for an exponential density profile $r_{1 / 2} \simeq 2.2 R_{\mathrm{d}}$, see Wolf et al. 2010), thus they may be slightly overestimated with respect to those of Irrs and BCDs (computed at $3.2 R_{\mathrm{d}}$ ), since the DM contribution is expected to increase at larger radii.

Figure 11 shows that starbursting dwarfs (open and filled circles) have baryonic fractions comparable with those of typical Irrs (grey dots) and of some Sphs (open diamonds). The Sphs with extremely low baryonic fractions $\left(f_{\text {bar }}<0.1\right)$ are Carina, Draco, Sextans and Ursa Minor, that are very close to the Milky Way and may have suffered from environmental effects (e.g., Mayer et al. 2006; Gatto et al. 2013). We conclude that the baryonic content of BCDs is similar to that of other types of dwarf galaxies, except for some low-luminosity satellities of the Milky Way.

\subsection{Stellar feedback and gas outflows}

Simulations of galaxy formation in a $\Lambda \mathrm{CDM}$ cosmology indicate that low-mass galaxies must experience massive gas outflows at high redshifts: this is necessary to reproduce the observed stellar and DM properties of dwarf galaxies at $z \simeq 0$ 

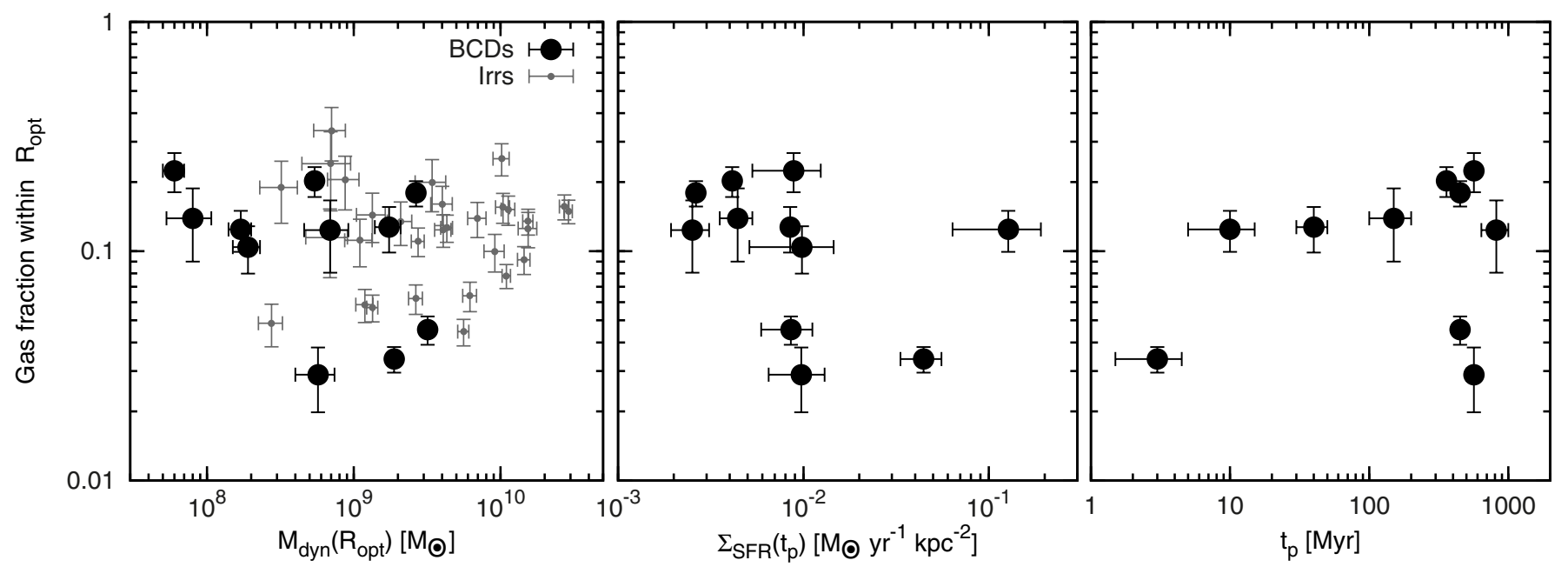

Fig. 12. Left: the atomic gas fractions $f_{\mathrm{gas}}=1.33 M_{\mathrm{HI}} / M_{\mathrm{dyn}}$ versus $M_{\mathrm{dyn}}$ calculated within the optical radius $R_{\mathrm{opt}}$. Large, black dots and small, grey dots indicate BCDs (this work) and Irrs (Swaters et al. 2009), respectively. Middle: $f_{\text {gas }}$ versus the SFR surface density $\Sigma_{S F R}\left(t_{\mathrm{p}}\right)=S F R_{\mathrm{p}} /\left(\pi R_{\mathrm{opt}}^{2}\right)$ where $\mathrm{SFR}_{\mathrm{p}}$ is the peak SFR over the last $1 \mathrm{Gyr}$ (see Table 1). Right: $f_{\text {gas }}$ versus the look-back time $t_{\mathrm{p}}$ at $\mathrm{SFR}$.

(e.g., Governato et al. 2010, 2012; Sawala et al. 2012) as well as their number density (e.g., Okamoto et al. 2010; Sawala et al. 2013). BCDs in the Local Universe can be considered as good analogues of dwarf galaxies at high redshifts, since they have small potential wells (with $20 \lesssim V_{\text {circ }} \lesssim 80 \mathrm{~km} \mathrm{~s}^{-1}$ ) and are currently experiencing intense star formation, as indicated by their recent SFHs ( $\lesssim 1 \mathrm{Gyr})$ and high values of the birthrate parameter $b$ (see Table 1 and McQuinn et al. 2010a). Moreover, starbursting dwarfs at $z \simeq 0$ have clumpy morphologies that are remarkably similar to those of more massive star-forming galaxies at $z \simeq 1-2$ (see e.g., Fig. 20 of Elmegreen et al. 2009), further suggesting a similarity with high- $z$ star-forming objects. While starburst galaxies are common at high redshifts, BCDs are relatively rare in the Local Universe: Lee et al. (2009) analyzed $\mathrm{H} \alpha$ observations from the 11HUGS survey (Kennicutt et al. 2008) and estimated that BCDs constitute only $\sim 6 \%$ of the population of star-forming dwarfs at $z \simeq 0$ (but see McQuinn et al. 2010a regarding the limitations of $\mathrm{H} \alpha$ observations to identify starbursting dwarfs). BCDs, therefore, offer us the opportunity to study in detail the actual importance of stellar feedback and gas outflows in low-mass DM halos.

We computed atomic gas fractions $f_{\text {gas }}=1.33 M_{\mathrm{HI}} / M_{\mathrm{dyn}}$ within $R_{\mathrm{opt}}$ for the $11 \mathrm{BCDs}$ with accurate estimates of $M_{\mathrm{dyn}}$ (see Sect. 7.1) and for the 30 Irrs considered in Sect. 8.1 (from Swaters et al. 2009). Fig. 12 (left panel) shows that both Irrs and BCDs have relatively high gas fractions $\left(0.1 \lesssim f_{\text {gas }} \lesssim 0.3\right)$, and only a few objects show gas fractions $\$ 5 \%$. Moreover, the gas fractions of BCDs are similar to those of Irrs. This suggests that either i) BCDs did not expell a large amount of gas out of their potential wells; or ii) their gas fractions were much higher at the beginning of the starburst; or iii) the gas expelled has been replenished by gas inflows. These three hypothesis are discussed in the following.

The possibility that massive gas inflows replenish the presumed outflowing gas seems unlikely, as we found evidence for radial motions in only four galaxies (see Sect. 6 and Lelli et al. 2012a,b). We do not know the direction of these radial motions, but if we interpret them as inflows, the inferred gas inflow rates would be only $\sim 1$ order of magnitude higher than the current SFRs and, thus, consistent with a typical efficiency of $\sim 10 \%$ in converting gas into stars. Moreover, it is likely that these radial motions are recent and short-lived phenomena, as their typical timescales are comparable with the orbital times (Lelli et al. $2012 b)$. For the other BCDs with a regularly rotating disk, any radial motion must be $\lesssim 5 \mathrm{~km} \mathrm{~s}^{-1}$, which gives us a firm upperlimit to the mean gas inflow rate of $\sim 0.3 M_{\odot} \mathrm{yr}^{-1}$. This seems adequate to feed the current star formation and build up the central concentration of gas observed in BCDs (see Fig. 2), but leave little room for massive gas outflows.

In Fig. 12 (middle panel), we plot $f_{\text {gas }}$ versus the SFR surface density $\Sigma_{\mathrm{SFR}}\left(t_{\mathrm{p}}\right)=S F R_{\mathrm{p}} /\left(\pi R_{\mathrm{opt}}^{2}\right)$, where $\mathrm{SFR}_{\mathrm{p}}$ is the peak SFR over the past $1 \mathrm{Gyr}$ (see Table 1). The SFHs of five galaxies (NGC 2366, NGC 4068, UGC 4483, UGC 9128, and SBS 1415+437) show two distinct peaks with similar SFRs (consistent within $1 \sigma$ ). Here we consider the older peak as this is the one that formed more stars, given that the SFR is averaged over a larger time-bin (typically a factor of $\sim 4$, see McQuinn et al. 2010a). One may expect that BCDs with higher values of $\Sigma_{\mathrm{SFR}}\left(t_{\mathrm{p}}\right)$ expell a higher fraction of gas out of their potential well and, thus, might have lower values of $f_{\text {gas }}$. This is not observed for the 11 objects considered here. However, in some BCDs the starburst started only $\sim 10 \mathrm{Myr}$ ago whereas in other ones it started more than 500 Myr ago (see e.g., McQuinn et al. 2010a). In Fig. 12 (right panel), we also plot $f_{\text {gas }}$ versus the lookback time $t_{\mathrm{p}}$ at $\mathrm{SFR}_{\mathrm{p}}$, which can be considered as the typical "age" of the starburst. There is also no clear correlation between these two quantities. Similarly, we found no clear correlation between $f_{\text {gas }}$ and the product $\Sigma_{\mathrm{SFR}} \times t_{\mathrm{p}}$ or other parameters that quantify the relative strength of the burst, such as $S F R_{\mathrm{p}} / M_{\text {dyn }}$, $S F R_{\mathrm{p}} / M_{*}$, and the birthrate parameter $b$ (see Table 1). The lack of any significant trend between $f_{\text {gas }}$ and the starburst properties suggests that BCDs did not eject a large quantity of gas out of their potential wells.

To further investigate the possible effects of outflows, we estimated the gas masses that might have been expelled from the potential wells of BCDs by using i) the escape velocities derived from the $\mathrm{HI}$ kinematics; ii) the burst energies derived from the observed SFHs; and iii) the feedback efficiencies derived by Mac Low \& Ferrara (1999) and Ferrara \& Tolstoy (2000) using both analytical calculations and high-resolution hydrodynamical simulations. Adopting Eq. (28) of Ferrara \& Tolstoy (2000), the mass-loss rate $\dot{M}_{\text {out }}$ due to stellar feedback is given by

$\dot{M}_{\text {out }}=2 \xi \eta E \gamma / V_{\text {out }}^{2}$, 
F. Lelli et al.: Dynamics of starbursting dwarf galaxies. III.

Table 6. Ouflowing gas masses from Eq. 8.

\begin{tabular}{lcccc}
\hline \hline Galaxy & $\begin{array}{c}\log \left(E_{\text {burst }}\right) \\
(\mathrm{erg})\end{array}$ & $\begin{array}{c}M_{\text {out }}^{\max } \\
\left(10^{6} M_{\odot}\right)\end{array}$ & $\begin{array}{c}\left\langle\dot{M}_{\text {out }}^{\max }\right\rangle \\
\left(M_{\odot} \mathrm{yr}^{-1}\right)\end{array}$ & $M_{\text {out }}^{\max } / M_{\text {gas }}$ \\
\hline NGC 625 & 56.0 & $11.2 \pm 1.9$ & 0.025 & 0.09 \\
NGC 1569 & 56.6 & $16.0 \pm 1.6$ & 0.036 & 0.04 \\
NGC 2366 & 56.5 & $12.2 \pm 0.5$ & 0.027 & 0.01 \\
NGC 4068 & 56.0 & $7.8 \pm 0.4$ & 0.017 & 0.04 \\
NGC 4214 & 56.7 & $8.1 \pm 0.4$ & 0.010 & 0.01 \\
NGC 6789 & 55.5 & $0.9 \pm 0.1$ & 0.002 & 0.04 \\
UGC 4483 & 55.4 & $5.7 \pm 0.5$ & 0.007 & 0.14 \\
UGC 9128 & 55.5 & $5.5 \pm 0.9$ & 0.004 & 0.32 \\
\hline
\end{tabular}

Notes. The burst energies are taken from McQuinn et al. (2010b). $M_{\text {out }}^{\max }$ is calculated assuming a feedback efficiency $\xi \times \eta \simeq 2 \times 10^{-3}$. See Sect. 8.2 for details.

where $\eta$ and $\xi$ are parameters related to the feedback efficiency, $E$ is the energy produced by supernovae and stellar winds, $\gamma$ is the energy production rate, and $V_{\text {out }}^{2}$ is the velocity of the outflowing gas that must be higher than the escape velocity $V_{\text {esc }}$. The parameter $\eta$ represents the fraction of injected energy that is converted into kinetic energy; for radiative bubbles $\eta \lesssim 0.03$ (Koo \& McKee 1992; Ferrara \& Tolstoy 2000). Since part of the kinetic energy accelerates material in the equatorial plane of the bubble, the parameter $\xi$ corresponds to the fraction of gas that is expelled from the galaxy almost perpendicular to the disk. Using hydrodynamical simulations, Mac Low \& Ferrara (1999) found that $\xi$ is usually $\lesssim 7 \%$. Following Ferrara \& Tolstoy (2000), we here assume that $\eta=0.03$ and $\xi=0.07$, but we warn that the actual values of these parameters are uncertain. Maximum massloss rates occur when $V_{\text {out }}=V_{\text {esc }}$. Assuming that $V_{\text {esc }}$ does not significantly change during the burst, the maximum mass that can be expelled from the galaxy is given by

$M_{\text {out }}^{\max }=\int_{t_{i}}^{t_{f}} \dot{M}_{\text {out }} \mathrm{d} t=2 \xi \eta V_{\mathrm{esc}}^{-2} \int_{t_{i}}^{t_{f}} E \gamma \mathrm{d} t=2 \xi \eta V_{\mathrm{esc}}^{-2} E_{\text {burst }}$,

where $t_{i}$ and $t_{f}$ are the initial and final times of the burst, and $E_{\text {burst }}$ is the total energy created during the burst. In Table 6, we list $M_{\text {out }}^{\max }$ for seven galaxies in our sample that have both good estimates of $E_{\text {burst }}$ and $V_{\text {esc }}$. We adopt the values of $E_{\text {burst }}$ calculated by McQuinn et al. (2010b) using the observed SFHs and the evolutionary synthesis code STARBURST99, which can simulate the energy produced by supernovae and stellar winds for a given SFR. We calculate $V_{\text {esc }}$ as $\sqrt{2} V_{\text {circ }}$ at the optical radius. Table 6 shows that, for $\eta \times \xi \simeq 2 \times 10^{-3}, M_{\text {out }}^{\max }$ is very small, less than $10 \%$ of the current atomic gas mass. Exceptions are UGC 4483 and UGC 9128, which are among the lowest mass galaxies in our sample, with rotation velocities of only $\sim 20 \mathrm{~km} \mathrm{~s}^{-1}$. Only if one assumes that $\eta \times \xi$ is a few times $10^{-2}$, the starbursting dwarfs in our sample could have expelled a gas mass comparable to the current, atomic gas mass.

Relatively low values of $M_{\text {out }} / M_{\text {gas }}$ are in line with the results of both optical and X-ray observations. Studies of the $\mathrm{H} \alpha$ kinematics have shown that galactic winds are common in BCDs, but the velocities of the $\mathrm{H} \alpha$ gas are usually smaller than the escape velocities (Martin 1996, 1998; van Eymeren et al. 2009b,a, 2010), implying that the ionized gas is gravitationally bound to the galaxy. Similar results have been found by studies of the Na D absorption doublet (Schwartz \& Martin 2004). X-ray observations have revealed that several starbursting dwarfs have a diffuse coronae of hot gas at $T \simeq 10^{6} \mathrm{~K}$, which are likely due to outflows but have low masses, $\sim 1 \%$ of the current H I mass (Ott et al. 2005a,b). The observational evidence, therefore, suggests that galactic winds are common in nearby dwarf galaxies, but they do not expell a significant fraction of the gas mass out of the potential well.

\section{Summary and conclusions}

We presented a systematic study of the H I content of 18 starbursting dwarf galaxies, using both new and archival observations. We only selected nearby galaxies that have been resolved into single stars by HST, thus providing information on their total stellar masses. According to their H I distribution and kinematics, we classified starbursting dwarfs into three main families: i) galaxies with a regularly rotating $\mathrm{H} \mathrm{I}$ disk $(\sim 50 \%)$; ii) galaxies with a kinematically disturbed H I disk ( $\sim 40 \%)$; and iii) galaxies with unsettled H I distributions ( $\sim 10 \%)$. For galaxies with a regularly rotating $\mathrm{H}$ I disk, we derived rotation curves by building 3D kinematic models. For galaxies with a kinematically disturbed H I disk, we obtained estimates of the rotation velocities in the outer parts. Our main results can be summarized as follows:

1. We firmly establish that the HI surface density profiles of starbursting dwarfs are different from those of typical Irrs. On average, starbursting dwarfs have central H I densities a factor of $\sim 2$ higher than typical Irrs.

2. The average ratio of the H I radius to the optical radius (defined as 3.2 exponential scale lengths) is $1.7 \pm 0.5$, similar to the values found for gas-rich spiral and irregular galaxies.

3. Disturbed HI kinematics are much more common in starbursting dwarfs $(\sim 50 \%)$ than in typical Irrs $(\sim 10 \%$, see Swaters et al. 2009). This may be related to stellar feedback and/or to the starburst trigger (interactions/mergers or disk instabilities).

4. Two galaxies (NGC 5253 and UGC 6456) show a velocity gradient along the $\mathrm{HI}$ minor axis. We modelled the $\mathrm{HI}$ emission by a disk dominated by radial motions and derived inflow/outflow timescales of $\sim 100-200$ Myr. For NGC 5253, the radial motions appear to be an inflow and would imply a gas inflow rate $\sim 1$ order of magnitude higher than the current SFR.

5. For 11 galaxies with accurate estimates of the circular velocities, we calculated the baryonic fraction $f_{\text {bar }}$ within the optical radius, using the stellar masses from the HST studies of the resolved stellar populations. We found that, on average, $f_{\text {bar }} \simeq 0.3$ for a Kroupa IMF and $f_{\text {bar }} \simeq 0.4$ for a Salpeter IMF. If molecular gas is also taken into account, the mean baryonic fraction may increase up to $\sim 0.5$.

6. For four galaxies with a regularly rotating $\mathrm{HI}$ disk centered on the stellar component, we decomposed the rotation curves into mass components. We found that baryons (both stars and gas) are generally not sufficient to explain the inner rise of the rotation curve, although they constitute $\sim 20-40 \%$ of the total mass at $\sim 2.2$ exponential scale lengths.

7. Despite the star formation having injected $\sim 10^{56}$ ergs into the ISM in the last $~ 500 \mathrm{Myr}$ (McQuinn et al. 2010b), these starbursting dwarfs have gas fractions comparable with those of typical Irrs. This suggests that either starbursting dwarfs do not expell a large amount of gas out of their potential wells, or their gas fractions must have been much higher at the beginning of the burst. The lack of any correlation between the 
observed gas fractions and the starburst properties favors the former scenario.

Acknowledgements. We are grateful to Renzo Sancisi for sharing insights and ideas that fueled this work. We thank Eline Tolstoy for stimulating discussions. We also thank Ed Elson and Angel R. Lopez-Sanchez for providing us with the HI datacubes of NGC 1705 and NGC 5253, respectively, and Polychronis Papaderos for the $\mathrm{H} \alpha$-subtracted HST image of I Zw 18. We finally thank the members of the WHISP, THINGS, and LITTLE-THINGS projects for having made the $\mathrm{HI}$ datacubes publicly available. F.L. acknowledges the Ubbo Emmius bursary program of the University of Groningen and the Leids KerkhovenBosscha Fund. F.F. acknowledges financial support from PRIN MIUR 20102011, project "The Chemical and Dynamical Evolution of the Milky Way and Local Group Galaxies", prot. 2010LY5N2T.

\section{References}

Annibali, F., Greggio, L., Tosi, M., Aloisi, A., \& Leitherer, C. 2003, AJ, 126, 2752

Annibali, F., Cignoni, M., Tosi, M., et al. 2013, AJ, 146, 144

Ashley, T., Simpson, C. E., \& Elmegreen, B. G. 2013, AJ, 146, 42

Baldwin, J. E., Lynden-Bell, D., \& Sancisi, R. 1980, MNRAS, 193, 313

Begeman, K. G. 1987, Ph.D. Thesis, Kapteyn Institute

Berg, D. A., Skillman, E. D., Marble, A. R., et al. 2012, ApJ, 754, 98

Bershady, M. A., Martinsson, T. P. K., Verheijen, M. A. W., et al. 2011, ApJ, 739, L47

Bigiel, F., Leroy, A., Walter, F., et al. 2008, AJ, 136, 2846

Binggeli, B., \& Popescu, C. C. 1995, A\&A, 298, 63

Boselli, A., Lequeux, J., \& Gavazzi, G. 2002, A\&A, 384, 33

Bravo-Alfaro, H., Brinks, E., Baker, A. J., Walter, F., \& Kunth, D. 2004, AJ, 127, 264

Briggs, D. S. 1995, in BAAS, 27, 1444

Broeils, A. H., \& Rhee, M.-H. 1997, A\&A, 324, 877

Brook, C. B., Governato, F., Roškar, R., et al. 2011, MNRAS, 415, 1051

Cannon, J. M., McClure-Griffiths, N. M., Skillman, E. D., \& Côté, S. 2004, ApJ, 607,274

Cannon, J. M., Most, H. P., Skillman, E. D., et al. 2011, ApJ, 735, 36

Côté, S., Carignan, C., \& Freeman, K. C. 2000, AJ, 120, 3027

Crone, M. M., Schulte-Ladbeck, R. E., Greggio, L., \& Hopp, U. 2002, ApJ, 567, 258

Dale, D. A., Cohen, S. A., Johnson, L. C., et al. 2009, ApJ, 703, 517

de Boer, T. J. L., Tolstoy, E., Hill, V., et al. 2012a, A\&A, 539, A103

de Boer, T. J. L., Tolstoy, E., Hill, V., et al. 2012b, A\&A, 544, A73

Elmegreen, D. M., Elmegreen, B. G., Marcus, M. T., et al. 2009, ApJ, 701, 306

Elson, E. C., de Blok, W. J. G., \& Kraan-Korteweg, R. C. 2010, MNRAS, 404, 2061

Elson, E. C., de Blok, W. J. G., \& Kraan-Korteweg, R. C. 2011, MNRAS, 415, 323

Elson, E. C., de Blok, W. J. G., \& Kraan-Korteweg, R. C. 2013, MNRAS, 429, 2550

Ferrara, A., \& Tolstoy, E. 2000, MNRAS, 313, 291

Fraternali, F., van Moorsel, G., Sancisi, R., \& Oosterloo, T. 2002, AJ, 123, 3124

Freeman, K. C. 1970, ApJ, 160, 811

Gallagher, III, J. S., \& Hunter, D. A. 1987, AJ, 94, 43

Gatto, A., Fraternali, F., Read, J. I., et al. 2013, MNRAS, 433, 2749

Gentile, G., Burkert, A., Salucci, P., Klein, U., \& Walter, F. 2005, ApJ, 634, L145

Gil de Paz, A., \& Madore, B. F. 2005, ApJS, 156, 345

Gil de Paz, A., Madore, B. F., \& Pevunova, O. 2003, ApJS, 147, 29

Governato, F., Brook, C., Mayer, L., et al. 2010, Nature, 463, 203

Governato, F., Zolotov, A., Pontzen, A., et al. 2012, MNRAS, 422, 1231

Guseva, N. G., Papaderos, P., Izotov, Y. I., et al. 2003, A\&A, 407, 105

Högbom, J. A. 1974, A\&AS, 15, 417

Hunter, D. A., \& Elmegreen, B. G. 2006, ApJS, 162, 49

Hunter, D. A., van Woerden, H., \& Gallagher, III, J. S. 1996, ApJS, 107, 739

Hunter, D. A., Wilcots, E. M., van Woerden, H., Gallagher, J. S., \& Kohle, S. 1998, ApJ, 495, L47

Hunter, D. A., van Woerden, H., \& Gallagher, J. S. 1999, AJ, 118, 2184

Hunter, D. A., Elmegreen, B. G., \& van Woerden, H. 2001, ApJ, 556, 773

Hunter, D. A., Ficut-Vicas, D., Ashley, T., et al. 2012, AJ, 144, 134

Izotov, Y. I., \& Thuan, T. X. 1999, ApJ, 511, 639

Johnson, M., Hunter, D. A., Oh, S.-H., et al. 2012, AJ, 144, 152

Kennicutt, Jr., R. C. 1998, ARA\&A, 36, 189

Kennicutt, Jr., R. C., Lee, J. C., Funes, J. G., S. J., Sakai, S., \& Akiyama, S. 2008, ApJS, 178, 247

Kobulnicky, H. A., \& Skillman, E. D. 1997, ApJ, 489, 636

Kobulnicky, H. A., \& Skillman, E. D. 2008, AJ, 135, 527

Koo, B.-C., \& McKee, C. F. 1992, ApJ, 388, 93
Kuchinski, L. E., Freedman, W. L., Madore, B. F., et al. 2000, ApJS, 131, 441

Lauberts, A., \& Valentijn, E. A. 1989, The surface photometry catalogue of the ESO-Uppsala galaxies

Lee, J. C., Kennicutt, Jr., R. C., Funes, S. J. J. G., Sakai, S., \& Akiyama, S. 2009 ApJ, 692, 1305

Lelli, F., Fraternali, F., \& Verheijen, M. 2014, A\&A, 563, A27

Lelli, F., Verheijen, M., Fraternali, F., \& Sancisi, R. 2012a, A\&A, 537, A72

Lelli, F., Verheijen, M., Fraternali, F., \& Sancisi, R. 2012b, A\&A, 544, A145

Leroy, A. K., Walter, F., Brinks, E., et al. 2008, AJ, 136, 2782

López-Sánchez, Á. R., Koribalski, B. S., van Eymeren, J., et al. 2012, MNRAS, 419,1051

Mac Low, M., \& Ferrara, A. 1999, ApJ, 513, 142

Marlowe, A. T., Meurer, G. R., \& Heckman, T. M. 1999, ApJ, 522, 183

Martin, C. L. 1996, ApJ, 465, 680

Martin, C. L. 1998, ApJ, 506, 222

Martinsson, T. P. K. 2011, Ph.D. Thesis, University of Groningen

Matthews, L. D., \& Uson, J. M. 2008, AJ, 135, 291

Mayer, L., Mastropietro, C., Wadsley, J., Stadel, J., \& Moore, B. 2006, MNRAS, 369,1021

McGaugh, S. S. 2005, ApJ, 632, 859

McGaugh, S. S. 2011, Phys. Rev. Lett., 106, 121303

McGaugh, S. S. 2012, AJ, 143, 40

McGaugh, S. S., Schombert, J. M., Bothun, G. D., \& de Blok, W. J. G. 2000, ApJ, 533, L99

McQuinn, K. B. W., Skillman, E. D., Cannon, J. M., et al. 2010a, ApJ, 721, 297

McQuinn, K. B. W., Skillman, E. D., Cannon, J. M., et al. 2010b, ApJ, 724, 49

McQuinn, K. B. W., Skillman, E. D., Dalcanton, J. J., et al. 2012, ApJ, 751, 127

Meurer, G. R., Freeman, K. C., Dopita, M. A., \& Cacciari, C. 1992, AJ, 103, 60

Meurer, G. R., Carignan, C., Beaulieu, S. F., \& Freeman, K. C. 1996, AJ, 111, 1551

Meurer, G. R., Staveley-Smith, L., \& Killeen, N. E. B. 1998, MNRAS, 300, 705

Meurer, G. R., Hanish, D. J., Ferguson, H. C., et al. 2006, ApJS, 165, 307

Oh, S., de Blok, W. J. G., Walter, F., Brinks, E., \& Kennicutt, R. C. 2008, AJ, 136,2761

Oh, S.-H., Brook, C., Governato, F., et al. 2011a, AJ, 142, 24

Oh, S.-H., de Blok, W. J. G., Brinks, E., Walter, F., \& Kennicutt, Jr., R. C. 2011b, AJ, 141, 193

Okamoto, T., Frenk, C. S., Jenkins, A., \& Theuns, T. 2010, MNRAS, 406, 208

Ott, J., Walter, F., \& Brinks, E. 2005a, MNRAS, 358, 1423

Ott, J., Walter, F., \& Brinks, E. 2005b, MNRAS, 358, 1453

Papaderos, P., Loose, H., Fricke, K. J., \& Thuan, T. X. 1996, A\&A, 314, 59

Papaderos, P., Izotov, Y. I., Thuan, T. X., et al. 2002, A\&A, 393, 461

Ramya, S., Kantharia, N. G., \& Prabhu, T. P. 2011, ApJ, 728, 124

Recchi, S., Matteucci, F., D’Ercole, A., \& Tosi, M. 2004, A\&A, 426, 37

Romano, D., Tosi, M., \& Matteucci, F. 2006, MNRAS, 365, 759

Roychowdhury, S., Chengalur, J. N., Kaisin, S. S., Begum, A., \& Karachentsev, I. D. 2011, MNRAS, 414, L55

Sackett, P. D. 1997, ApJ, 483, 103

Sánchez-Janssen, R., Méndez-Abreu, J., \& Aguerri, J. A. L. 2010, MNRAS, 406, L65

Sancisi, R., Fraternali, F., Oosterloo, T., \& van der Hulst, T. 2008, A\&A Rev., 15,189

Sawala, T., Scannapieco, C., \& White, S. 2012, MNRAS, 420, 1714

Sawala, T., Frenk, C. S., Crain, R. A., et al. 2013, MNRAS, 431, 1366

Schulte-Ladbeck, R. E., Hopp, U., Greggio, L., \& Crone, M. M. 2000, AJ, 120, 1713

Schulte-Ladbeck, R. E., Hopp, U., Greggio, L., Crone, M. M., \& Drozdovsky, I. O. 2001, AJ, 121, 3007

Schwartz, C. M., \& Martin, C. L. 2004, ApJ, 610, 201

Simpson, C. E., \& Gottesman, S. T. 2000, AJ, 120, 2975

Simpson, C. E., Hunter, D. A., Nordgren, T. E., et al. 2011, AJ, 142, 82

Stil, J. M., \& Israel, F. P. 2002, A\&A, 392, 473

Stringer, M. J., Bower, R. G., Cole, S., Frenk, C. S., \& Theuns, T. 2012, MNRAS, 423, 1596

Swaters, R. A., \& Balcells, M. 2002, A\&A, 390, 863

Swaters, R. A., van Albada, T. S., van der Hulst, J. M., \& Sancisi, R. 2002, A\&A, 390,829

Swaters, R. A., Sancisi, R., van Albada, T. S., \& van der Hulst, J. M. 2009, A\&A, 493, 871

Swaters, R. A., Sancisi, R., van Albada, T. S., \& van der Hulst, J. M. 2011, ApJ, 729,118

Taylor, C. L., Brinks, E., Pogge, R. W., \& Skillman, E. D. 1994, AJ, 107, 971

Taylor, C. L., Brinks, E., Grashuis, R. M., \& Skillman, E. D. 1995, ApJS, 99, 427

Taylor, C. L., Kobulnicky, H. A., \& Skillman, E. D. 1998, AJ, 116, 2746 
F. Lelli et al.: Dynamics of starbursting dwarf galaxies. III.

Taylor, V. A., Jansen, R. A., Windhorst, R. A., Odewahn, S. C., \& Hibbard, J. E. 2005, ApJ, 630, 784

Terlevich, R., Melnick, J., Masegosa, J., Moles, M., \& Copetti, M. V. F. 1991, A\&AS, 91, 285

Thuan, T. X., \& Izotov, Y. I. 2005, ApJS, 161, 240

Thuan, T. X., Hibbard, J. E., \& Lévrier, F. 2004, AJ, 128, 617

van Albada, T. S., \& Sancisi, R. 1986, Roy. Soc. London Philos. Trans. Ser. A, 320,447

van der Hulst, J., Terlouw, J., Begeman, K., Zwitser, W., \& Roelfsema, P. 1992, in ASP Conf. Ser. 25, eds. D. M. Worall, C. Biemesderfer, \& J. Barnes, 131 van der Kruit, P. C., \& Searle, L. 1981, A\&A, 95, 105

van Eymeren, J., Marcelin, M., Koribalski, B. S., et al. 2009a, A\&A, 505, 105

van Eymeren, J., Marcelin, M., Koribalski, B., et al. 2009b, A\&A, 493, 511 van Eymeren, J., Koribalski, B. S., López-Sánchez, Á. R., Dettmar, R.-J., \& Bomans, D. J. 2010, MNRAS, 407, 113

van Zee, L., Skillman, E. D., \& Salzer, J. J. 1998, AJ, 116, 1186

van Zee, L., Salzer, J. J., \& Skillman, E. D. 2001, AJ, 122, 121

Verheijen, M. A. W. 2001, ApJ, 563, 694

Verheijen, M., \& Sancisi, R. 2001, A\&A, 370, 765

Viallefond, F., \& Thuan, T. X. 1983, ApJ, 269, 444

Walter, F., Brinks, E., Duric, N., \& Klein, U. 1997, AJ, 113, 2031

Walter, F., Brinks, E., de Blok, W. J. G., et al. 2008, AJ, 136, 2563

Westfall, K. B., Bershady, M. A., Verheijen, M. A. W., et al. 2011, ApJ, 742, 18 Wilcots, E. M., \& Miller, B. W. 1998, AJ, 116, 2363

Wolf, J., Martinez, G. D., Bullock, J. S., et al. 2010, MNRAS, 406, 1220

Zibetti, S., Charlot, S., \& Rix, H.-W. 2009, MNRAS, 400, 1181 


\section{Appendix A: Notes on individual galaxies}

\section{A.1. Galaxies with a regularly rotating $\mathrm{HI}$ disk}

NGC 1705 has a strongly warped H I disk. Our rotation curve rises more steeply than those of Meurer et al. (1998) and Elson et al. (2013) because we applied a beam-smearing correction to the inner velocity-points using 3D disk models (see Fig. 5). Meurer et al. (1998) and Elson et al. (2013) decomposed their rotation curves into mass components and found that DM dominates the gravitational potential at all radii. We did not build a detailed mass model because the optical and kinematic centers differ by $\sim 550 \mathrm{pc}$, while $\mathrm{PA}_{\text {opt }}$ and $\mathrm{PA}_{\text {kin }}$ differ by $\sim 45^{\circ}$.

NGC 2366 has an extended H I disk with a strong kinematic distortion to the North-West (see its velocity field in Appendix C). Our rotation curve is in overall agreement with previous results (Hunter et al. 2001; Thuan et al. 2004; Oh et al. 2008; Swaters et al. 2009; van Eymeren et al. 2009b), but we do not confirm the declining part of the rotation curve found by Hunter et al. (2001) and van Eymeren et al. (2009b) at $R \gtrsim 5^{\prime}$. This latter result appears to be caused by an anomalous H I cloud that lies at $V_{\mathrm{los}} \simeq 130 \mathrm{~km} \mathrm{~s}^{-1}$ along the major axis $\left(\sim 7^{\prime}\right.$ from the galaxy center to the North, see the PV-diagram in Fig. 3 and Appendix C). NGC 4068 has a H I distribution characterized by a central depression and several shell-like structures. The HI kinematics is slightly lopsided. Our rotation curve agrees with the one of Swaters et al. (2009) within the errors.

NGC 4214 has a H I disk with multiple spiral arms. Intriguingly, the optical and HI spiral arms wind in opposite directions (clockwise and counter-clockwise, respectively). The H I disk is close to face-on and strongly warped, thus the rotation curve is uncertain. In the inner parts, our rotation curve rises more steeply than the one derived by Swaters et al. (2009); the difference seems to be due to a different choice of the dynamical center (see the PV-diagram in Swaters et al. 2009).

NGC 6789 has a compact H I disk that extends out to only $\sim 3.5$ optical scale lengths. The inclination is uncertain: we derived $i=43^{\circ} \pm 7^{\circ}$ using $3 \mathrm{D}$ disk models.

UGC 4483 has been studied in Lelli et al. (2012b).

I Zw 18 has been studied in Lelli et al. (2012a).

I Zw 36 has an extended and asymmetric H I distribution (see Ashley et al. 2013), but in the central parts the H I forms a compact, rotating disk. The optical and kinematic centers are offset by $\sim 12^{\prime \prime}(\sim 340 \mathrm{pc})$, while $\mathrm{PA}_{\text {opt }}$ and $\mathrm{PA}_{\text {kin }}$ differ by $\sim 36^{\circ}$.

SBS $1415+437$ is a prototype "cometary" BCD, as the starburst region is located at the edge of an elongated stellar body. Remarkably, the kinematic center does not coincide with the optical one but with the starburst region to the South (see Appendix $\mathrm{C}$; the object at $\mathrm{RA} \simeq 14^{\mathrm{h}} 17^{\mathrm{m}} 00^{\mathrm{s}}$ and $\mathrm{Dec} \simeq$ $43^{\circ} 29^{\prime} 45^{\prime \prime}$ is a foreground star). The lopsided H I distribution and kinematics may be due to a pattern of elliptical orbits centered on the starburst region (cf. Baldwin et al. 1980).

\section{A.2. Galaxies with a kinematically disturbed HI disk}

NGC 625 has been previously studied by Côté et al. (2000) and Cannon et al. (2004). Côté et al. (2000) suggested that the complex H I kinematics is due to an interaction/merger, whereas Cannon et al. (2004) argued that it is best described by a gaseous outflow superimposed on a rotating disk. We find it difficult to distinguish between these two possibilities. It is clear, however, that the galaxy has a inner, rotating disk with $V_{\text {rot }} \simeq 30 \mathrm{~km} \mathrm{~s}^{-1}$ (see PV-diagram in Appendix C).
NGC 1569 has been previously studied by Stil \& Israel (2002) and Johnson et al. (2012). Both studies derived a rotation curve by fitting the $\mathrm{HI}$ velocity field with a tilted-ring model. The PV-diagram along the major axis, however, does not show any sign of rotation in the inner parts $\left(R \lesssim 1^{\prime}\right.$, see Appendix $\mathrm{C}$ ). Moreover, the H I line-profiles are very broad and asymmetric, likely due to strong non-circular motions. For these reasons, we restrict our analysis to the rotation velocity in the outer parts $\left(\sim 50 \mathrm{~km} \mathrm{~s}^{-1}\right)$.

NGC 4163 shows a very small velocity gradient of $\sim 10 \mathrm{~km} \mathrm{~s}^{-1}$. The complex H I kinematics may be due to the low $V_{\text {rot }} / \sigma_{\mathrm{HI}}$ ratio. The PA of the stellar body and of the H I disk significantly differ by $\sim 40^{\circ}$.

NGC 4449 has been previously studied by Hunter et al. (1998, 1999), who found that the HI distribution forms 2 counterrotating systems. For the inner H I disk, we find a rotation velocity of $\sim 35 \mathrm{~km} \mathrm{~s}^{-1}$. It is unclear whether the outer gas system is really a counter-rotating disk or is formed by two or three $\mathrm{H} \mathrm{I}$ tails wrapping around the inner disk (similarly to I Zw 18, see Lelli et al. 2012a).

NGC 5253 has been previously studied by Kobulnicky \& Skillman (2008) and López-Sánchez et al. (2012), who discussed the possibility of gas inflows/outflows along the minor axis of the galaxy. The data are, indeed, consistent with a H I disk with $V_{\text {rot }}<5 \mathrm{~km} \mathrm{~s}^{-1}$ and $V_{\text {rad }} \simeq 25 \mathrm{~km} \mathrm{~s}^{-1}$ (see Fig. 7). Shadowing of the X-ray emission indicates that the southern side of the galaxy is the nearest one to the observer (Ott et al. 2005a), suggesting that the radial motions are an inflow.

UGC 6456 has been previously studied by Thuan et al. (2004) and Simpson et al. (2011). Simpson et al. (2011) derived a rotation curve using low-resolution $(\mathrm{C}+\mathrm{D}$ array) observations. They assumed different values of the PA for the approaching and receding sides, which would imply an unusual, asymmetric warp starting within the stellar component (see their Fig. 13). Our 3D models show that the HI kinematics may be simply explained by a disk with $V_{\text {rot }} \simeq V_{\text {rad }} \simeq \sigma_{\mathrm{HI}} \simeq 10 \mathrm{~km} \mathrm{~s}^{-1}$ (see Fig. 6).

UGC 9128 has a $\mathrm{HI}$ disk that rotates at $\sim 25 \mathrm{~km} \mathrm{~s}^{-1}$, but the VF is very irregular and the H I line profiles are broad and asymmetric, possibly due to non-circular motions. The optical and kinematic PA differ by $\sim 30^{\circ}$.

\section{A.3. Galaxies with unsettled HI distribution}

UGC 6541 has a very asymmetric H I distribution. Gas emission is detected only in the northern half of the galaxy. This may be the remnant of a disrupted disk.

UGCA 290 has a H I distribution that is offset with respect to the stellar component. The kinematics is irregular and dominated by a few distinct H I clouds.

\section{Appendix B: Tables}

\section{B.1. Properties of the $\mathrm{HI}$ datacubes}

Column (1) gives the galaxy name, following the ordering NGC, UGC, UGCA, Zwicky, SBS.

Column (2) gives the radio interferometer used for the $21 \mathrm{~cm}$ line observations.

Columns (3)-(5) give the spatial and spectral resolutions of the original cube. This cube is typically obtained using a Robust parameter $\mathfrak{R} \simeq 0$.

Columns (6)-(8) give the spatial and spectral resolutions of the cube after Gaussian smoothing.

Column (9) gives the ratio of the $\mathrm{HI}$ radius $R_{\mathrm{HI}}$ (given in Table 3) to the final H I beam. 
F. Lelli et al.: Dynamics of starbursting dwarf galaxies. III.

Table B.1. Properties of the H I datacubes.

\begin{tabular}{|c|c|c|c|c|c|c|c|c|c|c|}
\hline \multirow{3}{*}{$\begin{array}{l}\text { Name } \\
\text { (1) } \\
\end{array}$} & \multirow{3}{*}{$\begin{array}{l}\text { Telescope } \\
\text { (2) }\end{array}$} & \multicolumn{2}{|c|}{ Original beam } & \multirow{3}{*}{$\begin{array}{c}\text { Original } \Delta V \\
\left(\mathrm{~km} \mathrm{~s}^{-1}\right) \\
(5)\end{array}$} & \multicolumn{2}{|c|}{ Final beam } & \multirow{3}{*}{$\begin{array}{c}\text { Final } \Delta V \\
\left(\mathrm{~km} \mathrm{~s}^{-1}\right) \\
(8)\end{array}$} & \multirow{3}{*}{$\begin{array}{c}R_{\mathrm{HI}} / \text { beam } \\
(9) \\
(9)\end{array}$} & \multirow{3}{*}{$\begin{array}{c}\text { Rms noise } \\
(\mathrm{mJy} / \text { beam }) \\
(10)\end{array}$} & \multirow{3}{*}{$\begin{array}{r}\text { Source } \\
\text { (11) } \\
\end{array}$} \\
\hline & & $($ asec $\times$ asec $)$ & $(\mathrm{pc} \times \mathrm{pc})$ & & $($ asec $\times$ asec $)$ & $(\mathrm{pc} \times \mathrm{pc})$ & & & & \\
\hline & & (3) & (4) & & (6) & (7) & & & & \\
\hline NGC 625 & VLA & $18.9 \times 11.7$ & $357 \times 221$ & 2.6 & $30.0 \times 30.0$ & $567 \times 567$ & 5.2 & 4.6 & 1.80 & $\mathrm{a}$ \\
\hline NGC 1569 & VLA & $5.8 \times 5.0$ & $96 \times 82$ & 2.6 & $10.0 \times 10.0$ & $165 \times 165$ & 5.2 & 23.6 & 0.46 & $\mathrm{~b}$ \\
\hline NGC 1705 & ATCA & $16.7 \times 14.5$ & $413 \times 358$ & 4.0 & $16.7 \times 14.5$ & $413 \times 358$ & 7.0 & 5.5 & 0.40 & $\mathrm{c}$ \\
\hline NGC 2366 & VLA & $6.9 \times 5.9$ & $107 \times 91$ & 2.6 & $15.0 \times 15.0$ & $233 \times 233$ & 5.2 & 29.2 & 0.66 & $\mathrm{~b}$ \\
\hline NGC 4068 & WSRT & $14.8 \times 11.5$ & $308 \times 240$ & 2.5 & $20.0 \times 20.0$ & $417 \times 417$ & 6.1 & 7.4 & 2.00 & d \\
\hline NGC 4163 & VLA & $9.7 \times 5.9$ & $141 \times 86$ & 1.3 & $10.0 \times 10.0$ & $145 \times 145$ & 5.2 & 7.6 & 0.43 & $\mathrm{~b}$ \\
\hline NGC 4214 & VLA & $7.6 \times 6.4$ & $99 \times 84$ & 1.3 & $30.0 \times 30.0$ & $393 \times 393$ & 5.2 & 14.0 & 2.20 & $\mathrm{~b}$ \\
\hline NGC 4449 & VLA & $13.7 \times 12.5$ & $279 \times 254$ & 5.2 & $20.0 \times 20.0$ & $407 \times 407$ & 10.4 & 21.9 & 0.80 & $\mathrm{e}$ \\
\hline NGC 5253 & ATCA & $13.6 \times 7.5$ & $231 \times 127$ & 4.0 & $20.0 \times 20.0$ & $339 \times 339$ & 9.0 & 9.1 & 0.95 & $\mathrm{f}$ \\
\hline NGC 6789 & WSRT & $13.7 \times 12.7$ & $239 \times 222$ & 2.5 & $13.7 \times 12.7$ & $239 \times 222$ & 6.1 & 4.3 & 0.75 & $\mathrm{a}$ \\
\hline UGC 4483 & VLA & $5.7 \times 4.5$ & $88 \times 70$ & 2.6 & $10.0 \times 10.0$ & $155 \times 155$ & 5.2 & 9.0 & 0.66 & g \\
\hline UGC 6456 & VLA & $5.7 \times 4.8$ & $119 \times 100$ & 2.6 & $15.0 \times 15.0$ & $313 \times 313$ & 5.2 & 5.7 & 0.90 & a \\
\hline UGC 6541 & VLA & $6.2 \times 5.5$ & $126 \times 112$ & 1.3 & $10.0 \times 10.0$ & $204 \times 204$ & 5.2 & $\ldots$ & 0.44 & $\mathrm{~b}$ \\
\hline UGC 9128 & VLA & $6.2 \times 5.5$ & $66 \times 59$ & 2.6 & $15.0 \times 15.0$ & $160 \times 160$ & 5.2 & 5.6 & 0.80 & $\mathrm{~b}$ \\
\hline UGCA 290 & VLA & $5.4 \times 4.2$ & $175 \times 136$ & 1.9 & $10.0 \times 10.0$ & $325 \times 325$ & 4.9 & ... & 0.56 & a \\
\hline I Zw 18 & VLA & $1.5 \times 1.4$ & $132 \times 123$ & 1.3 & $5.0 \times 5.0$ & $441 \times 441$ & 5.2 & 7.5 & 0.16 & $\mathrm{~h}$ \\
\hline I Zw 36 & VLA & $6.8 \times 5.5$ & $194 \times 157$ & 2.6 & $6.8 \times 5.5$ & $194 \times 157$ & 5.2 & 10.9 & 0.34 & $\mathrm{~b}$ \\
\hline SBS $1415+437$ & VLA & $4.6 \times 4.3$ & $303 \times 283$ & 1.9 & $10.0 \times 10.0$ & $659 \times 659$ & 4.9 & 6.5 & 0.60 & $\mathrm{a}$ \\
\hline
\end{tabular}

References. (a) This work; (b) Hunter et al. (2012); (c) Elson et al. (2013); (d) Swaters et al. (2002); (e) Walter et al. (2008); (f) López-Sánchez et al. (2012); (g) Lelli et al. (2012b); (h) Lelli et al. (2012a).

Table B.2. Optical and H I orientation parameters.

\begin{tabular}{|c|c|c|c|c|c|c|c|c|c|c|c|}
\hline Name & $\begin{array}{c}\mathrm{RA}_{\mathrm{opt}} \\
(\mathrm{J} 2000) \\
(2) \\
\end{array}$ & $\begin{array}{c}\operatorname{Dec}_{\mathrm{opt}} \\
(\mathrm{J} 2000) \\
(3) \\
\end{array}$ & $\epsilon_{\mathrm{opt}}$ & $\begin{array}{l}i_{\mathrm{opt}} \\
\left({ }^{\circ}\right) \\
(5) \\
\end{array}$ & $\begin{array}{c}\mathrm{PA}_{\mathrm{opt}} \\
\left(^{\circ}\right) \\
(6) \\
\end{array}$ & $\begin{array}{c}\mathrm{RA}_{\text {kin }} \\
(\mathrm{J} 2000) \\
(7) \\
\end{array}$ & $\begin{array}{c}\text { Dec }_{\text {kin }} \\
(\mathrm{J} 2000) \\
(8) \\
\end{array}$ & $\begin{array}{c}V_{\text {sys }} \\
\left(\mathrm{km} \mathrm{s}^{-1}\right) \\
(9)\end{array}$ & $\begin{array}{c}i_{\text {kin }} \\
\left({ }^{\circ}\right) \\
(10) \\
\end{array}$ & $\begin{array}{c}\mathrm{PA}_{\mathrm{kin}} \\
\left(^{\circ}\right) \\
(11) \\
\end{array}$ & $\begin{array}{c}\Delta c \\
(\mathrm{pc}) \\
(12) \\
\end{array}$ \\
\hline \multicolumn{12}{|c|}{ Galaxies with a regularly rotating HI disk } \\
\hline NGC 1705 & 045413.9 & -532125 & 0.28 & $47 \pm 2$ & $55 \pm 3$ & 045416.1 & -532135 & $635 \pm 2$ & $45: 85$ & $10 \pm 5$ & $552 \pm 164$ \\
\hline NGC 2366 & 072851.9 & +691234 & 0.66 & $80 \pm 2$ & $29 \pm 4$ & 072853.3 & +691243 & $103 \pm 1$ & $68 \pm 5$ & $42 \pm 2$ & $150 \pm 99$ \\
\hline NGC 4068 & 120402.7 & +523528 & 0.38 & $56 \pm 4$ & $31 \pm 4$ & 120403.0 & +523530 & $206 \pm 2$ & $44 \pm 6$ & $24 \pm 3$ & 0 \\
\hline NGC 4214 & 121538.8 & +361939 & 0.09 & $26 \pm 5$ & $40 \pm 20$ & 121536.9 & +361959 & $291 \pm 1$ & $30:-1$ & $65: 84$ & $393 \pm 167$ \\
\hline NGC 6789 & 191641.9 & +635817 & 0.15 & $34 \pm 2$ & $86 \pm 4$ & 191641.9 & +635817 & $-151 \pm 2$ & $43 \pm 7$ & $82 \pm 5$ & 0 \\
\hline UGC 4483 & 083703.4 & +694631 & 0.47 & $63 \pm 3$ & $-13 \pm 5$ & 083703.4 & +694631 & $158 \pm 2$ & $58 \pm 3$ & $0 \pm 5$ & 0 \\
\hline I Zw 18 & 093402.0 & +551425 & 0.50 & $65 \pm 5$ & $135 \pm 1$ & 093402.0 & +551425 & $767 \pm 4$ & $70 \pm 4$ & $145 \pm 5$ & 0 \\
\hline I Zw 36 & 122616.8 & +482939 & 0.30 & $49 \pm 2$ & $80 \pm 3$ & 122618.0 & +482941 & $277 \pm 2$ & $67 \pm 3$ & $44 \pm 3$ & $340 \pm 74$ \\
\hline SBS $1415+437$ & 141702.1 & +433019 & 0.66 & $80 \pm 3$ & $30 \pm 5$ & 141701.7 & +433007 & $616 \pm 2$ & $66 \pm 3$ & $23 \pm 3$ & $824 \pm 280$ \\
\hline \multicolumn{12}{|c|}{ Galaxies with a kinematically disturbed H I disk } \\
\hline NGC 625 & 013504.3 & -412615 & 0.64 & $78 \pm 2$ & $94 \pm 1$ & 013506.3 & -412617 & $398 \pm 6$ & $\ldots$ & $120 \pm 10$ & 0 \\
\hline NGC 1569 & 043049.0 & +645053 & 0.54 & $69 \pm 2$ & $118 \pm 3$ & 043051.9 & +645056 & $-80 \pm 10$ & $\ldots$ & $115 \pm 10$ & $310 \pm 70$ \\
\hline NGC 4163 & 121209.0 & +361011 & 0.30 & $49 \pm 2$ & $14 \pm 2$ & 121209.0 & +361016 & $158 \pm 4$ & $\ldots$ & $-25 \pm 10$ & $72 \pm 62$ \\
\hline NGC 4449 & 122810.8 & +440537 & 0.40 & $57 \pm 3$ & $55 \pm 3$ & 122811.3 & +440558 & $210 \pm 5$ & $\ldots$ & $60 \pm 5$ & $444 \pm 173$ \\
\hline NGC 5253 & 133956.0 & -313831 & 0.53 & $68 \pm 2$ & $43 \pm 2$ & 133956.0 & -313831 & $410 \pm 10$ & $\ldots$ & $40 \pm 5$ & 0 \\
\hline UGC 6456 & 112757.2 & +785948 & 0.50 & $65 \pm 5$ & $-10 \pm 5$ & 112758.8 & +785951 & $-102 \pm 4$ & $\ldots$ & $0 \pm 5$ & 0 \\
\hline UGC 9128 & 141556.8 & +230322 & 0.28 & $47 \pm 6$ & $33 \pm 6$ & 141557.6 & +230308 & $150 \pm 4$ & $\ldots$ & $0 \pm 10$ & $181 \pm 68$ \\
\hline \multicolumn{12}{|c|}{ Galaxies with unsettled H I distribution } \\
\hline UGC 6541 & 113328.9 & +491422 & 0.50 & $65 \pm 4$ & $129 \pm 2$ & $\ldots$ & $\ldots$ & $250 \pm 2$ & $\ldots$ & $\ldots$ & $\ldots$ \\
\hline UGCA 290 & 123722.1 & +384441 & 0.50 & $65 \pm 3$ & $47 \pm 3$ & $\ldots$ & $\ldots$ & $468 \pm 5$ & $\ldots$ & $\ldots$ & $\ldots$ \\
\hline
\end{tabular}


Table B.3. Mass budget within the optical radius.

\begin{tabular}{|c|c|c|c|c|c|c|c|c|c|c|c|}
\hline Name & $M_{*}^{\mathrm{Sal}}$ & $M_{\text {mol }}$ & $\begin{array}{c}M_{\mathrm{HI}}\left(R_{\mathrm{opt}}\right) \\
\left(10^{7} M\right. \\
(4) \\
\end{array}$ & $\begin{array}{r}M_{\mathrm{bar}}^{\mathrm{Kr}} \\
(5) \\
\end{array}$ & $M_{\mathrm{bar}}^{\mathrm{Sal}}$ & $M_{\mathrm{bar}}^{\mathrm{mol}}$ & $\begin{array}{c}V_{\text {circ }}\left(R_{\mathrm{opt}}\right) \\
\left(\mathrm{km} \mathrm{s}^{-1}\right) \\
(8)\end{array}$ & $\begin{array}{c}M_{\mathrm{dyn}}\left(R_{\mathrm{opt}}\right) \\
\left(10^{7} M_{\odot}\right) \\
(9) \\
\end{array}$ & (10) & $f_{\mathrm{bar}}^{\mathrm{Sal}}$ & $f_{\text {bar }}^{\text {mol }}$ \\
\hline \multicolumn{12}{|c|}{ Galaxies with a regularly rotating HI disk } \\
\hline NGC 1705 & $>20$ & $60 \pm 18$ & $4.8 \pm 0.5$ & $>19$ & $>26$ & $>86$ & $73 \pm 3$ & $185 \pm 36$ & $>0.10$ & $>0.14$ & $>0.47$ \\
\hline NGC 2366 & $26 \pm 3$ & $8 \pm 2$ & $36 \pm 4$ & $64 \pm 5$ & $74 \pm 6$ & $82 \pm 6$ & $51 \pm 2$ & $263 \pm 28$ & $0.24 \pm 0.03$ & $0.28 \pm 0.04$ & $0.31 \pm 0.04$ \\
\hline NGC 4068 & $22 \pm 3$ & $5.9 \pm 1.8$ & $8.2 \pm 0.8$ & $25 \pm 2$ & $33 \pm 3$ & $39 \pm 4$ & $36 \pm 2$ & $54 \pm 13$ & $0.46 \pm 0.12$ & $0.61 \pm 0.16$ & $0.72 \pm 0.19$ \\
\hline NGC 4214 & $>28$ & $12 \pm 4$ & $11 \pm 1$ & $>32$ & $>42$ & $>54$ & $79 \pm 4$ & $325 \pm 104$ & $>0.10$ & $>0.13$ & $>0.17$ \\
\hline NGC 6789 & $7 \pm 2$ & $0.6 \pm 0.2$ & $1.2 \pm 0.1$ & $6.0 \pm 1.3$ & $8.6 \pm 2.0$ & $9.2 \pm 2.0$ & $59 \pm 9$ & $60 \pm 24$ & $0.10 \pm 0.05$ & $0.14 \pm 0.07$ & $0.15 \pm 0.07$ \\
\hline UGC 4483 & $1.0 \pm 0.2$ & $2.1 \pm 0.6$ & $1.0 \pm 0.1$ & $2.0 \pm 0.2$ & $2.3 \pm 0.2$ & $4.4 \pm 0.6$ & $21 \pm 2$ & $6.6 \pm 1.3$ & $0.30 \pm 0.07$ & $0.36 \pm 0.08$ & $0.68 \pm 0.17$ \\
\hline $\mathrm{I} Z \mathrm{Zw} 18$ & $>1.7$ & $7 \pm 2$ & $1.6 \pm 0.2$ & $>3.2$ & $>3.8$ & $>11$ & $38 \pm 4$ & $16 \pm 3$ & $>0.20$ & $>0.24$ & $>0.67$ \\
\hline I Zw 36 & $>0.8$ & $4.7 \pm 1.4$ & $1.5 \pm 0.1$ & $>2.5$ & $>2.8$ & $>7.5$ & $30 \pm 3$ & $19 \pm 4$ & $>0.13$ & $>0.14$ & $>0.38$ \\
\hline SBS $1415+437$ & $17 \pm 3$ & $7.6 \pm 2.3$ & $6.8 \pm 0.7$ & $20 \pm 2$ & $26 \pm 3$ & $34 \pm 4$ & $22 \pm 2$ & $27 \pm 5$ & $0.74 \pm 0.16$ & $0.98 \pm 0.22$ & $1.26 \pm 0.28$ \\
\hline \multicolumn{12}{|c|}{ Galaxies with a kinematically disturbed $\mathrm{H}$ I disk } \\
\hline NGC 625 & $26 \pm 10$ & $0.8 \pm 0.2$ & $6.4 \pm 0.6$ & $25 \pm 6$ & $34 \pm 10$ & $35 \pm 10$ & $30 \pm 5$ & $70 \pm 23$ & $0.36 \pm 0.15$ & $0.50 \pm 0.22$ & $0.51 \pm 0.22$ \\
\hline NGC 1569 & $70 \pm 7$ & $15 \pm 5$ & $17 \pm 2$ & $66 \pm 5$ & $92 \pm 7$ & $107 \pm 9$ & $50 \pm 5$ & $177 \pm 36$ & $0.37 \pm 0.08$ & $0.52 \pm 0.11$ & $0.61 \pm 0.13$ \\
\hline NGC 4163 & $10 \pm 3$ & $1.0 \pm 0.3$ & $1.1 \pm 0.1$ & $8 \pm 2$ & $11 \pm 3$ & $12 \pm 3$ & $10 \pm 4$ & $2.4 \pm 1.9$ & $3.3 \pm 2.7$ & $4.8 \pm 4.1$ & $5.2 \pm 4.4$ \\
\hline NGC 4449 & $210 \pm 35$ & $184 \pm 55$ & $32 \pm 3$ & $174 \pm 22$ & $252 \pm 35$ & $436 \pm 65$ & $35 \pm 5$ & $94 \pm 28$ & $1.9 \pm 0.6$ & $2.7 \pm 0.9$ & $4.6 \pm 1.5$ \\
\hline NGC 5253 & $154 \pm 21$ & $31 \pm 9$ & $6.8 \pm 0.7$ & $106 \pm 13$ & $163 \pm 21$ & $194 \pm 23$ & $<5$ & $\ldots$ & $\ldots$ & $\ldots$ & $\ldots$ \\
\hline UGC 6456 & $5 \pm 2$ & $4.3 \pm 1.3$ & $2.6 \pm 0.3$ & $6.6 \pm 1.3$ & $8.5 \pm 2.0$ & $13 \pm 2$ & $10 \pm 5$ & $\ldots$ & $\ldots$ & $\ldots$ & $\ldots$ \\
\hline UGC 9128 & $1.3 \pm 0.2$ & $0.13 \pm 0.04$ & $0.8 \pm 0.1$ & $2.0 \pm 0.2$ & $2.4 \pm 0.2$ & $2.5 \pm 0.2$ & $24 \pm 4$ & $7.7 \pm 3.0$ & $0.25 \pm 0.10$ & $0.31 \pm 0.12$ & $0.33 \pm 0.13$ \\
\hline \multicolumn{12}{|c|}{ Galaxies with unsettled HI distribution } \\
\hline UGC 6541 & $>0.8$ & $0.6 \pm 0.2$ & $1.2 \pm 0.1$ & $>2.1$ & $>2.4$ & $>2.7$ & $\ldots$ & $\ldots$ & $\ldots$ & $\ldots$ & $\ldots$ \\
\hline UGCA 290 & $>1$ & $2.1 \pm 0.6$ & $1.4 \pm 0.2$ & $>2.5$ & $>2.9$ & $>4.9$ & $\ldots$ & $\ldots$ & $\ldots$ & $\ldots$ & $\ldots$ \\
\hline
\end{tabular}

Column (10) gives the noise in the final cube.

Column (11) provides the reference for the original cube.

\section{B.2. Optical and H I orientation parameters}

Column (1) gives the galaxy name.

Columns (2)-(6) give the optical center, ellipticity, inclination, and position angle. These values are derived by interactively fitting ellipses to the outer isophotes. The inclination is calculated assuming an oblate spheroid with intrinsic thickness $q_{0}=0.3$.

Columns (7)-(11) give the kinematical center, systemic velocity, inclination, and position angle. These values are derived using HI velocity fields, channel maps, PV-diagrams, and building 3D disk models.

Column (12) gives the projected offset between the optical and kinematical centers. This is calculated as $\sqrt{\left(\alpha_{\text {opt }}-\alpha_{\text {kin }}\right)^{2}-\left(\delta_{\text {opt }}-\delta_{\text {kin }}\right)^{2}}$, assuming the galaxy distance given in Table 1. The error is estimated as $F W H M / 2.35$, where FWHM is the beam of the smoothed HI datacube (see Table B.1). Projected distances smaller than $F W H M / 2.35$ are assumed to be zero.

\section{B.3. Mass budget within the optical radius.}

Column (1) gives the galaxy name.

Column (2) gives the stellar mass. This is calculated by integrating the galaxy SFH and assuming a gas-recycling efficiency of $30 \%$. The SFHs were derived by fitting the CMDs of the resolved stellar populations and assuming a Salpeter IMF from 0.1 to $100 M_{\odot}$.
Column (3) gives the molecular mass. This is indirectly estimated using Eq. (4), which assumes that the star-formation efficiency in dwarfs is the same as in spirals.

Column (4) gives the H I mass $M_{\mathrm{HI}}$ within $R_{\mathrm{opt}}$.

Column (5)-(7) give the baryonic mass within $R_{\text {opt }}$ assuming, respectively, a Kroupa IMF, a Salpeter IMF, and a Salpeter IMF plus the possible contribution of molecules.

Column (8) gives the circular velocity at $R_{\mathrm{opt}}$.

Column (9) gives the dynamical mass within $R_{\mathrm{opt}}$ calculated as $M_{\text {dyn }}=V_{\text {circ }}^{2}\left(R_{\mathrm{opt}}\right) \times R_{\mathrm{opt}} / G$.

Columns (10)-(12) gives the baryonic fraction within $R_{\mathrm{opt}}$ assuming, respectively, a Kroupa IMF, a Salpeter IMF, and a Salpeter IMF plus molecules. Italics indicate unphysical values $>1$.

\section{Appendix C: Atlas}

In the following, we present overview figures for the 18 starbursting dwarfs in our sample. For each galaxy, we show six panels including both optical and H I data.

Top-left: a sky-subtracted optical image in the $R$ or $V$ band. The cross shows the optical center.

Bottom-left: an isophotal map (black contours) overlaid with a set of concentric ellipses (white contours). The value of the outermost isophote $\mu_{\text {out }}$ is given in the note; the isophotes increase in steps of $1 \mathrm{mag} \operatorname{arcsec}^{-2}$. The orientation parameters for the ellipses $\left(\epsilon_{\mathrm{opt}}\right.$ and $\left.\mathrm{PA}_{\mathrm{opt}}\right)$ are given in Table B.2. The cross shows the optical center. For I Zw 18, the isophotal map was derived from a $R$-band HST image after the subtraction of the $\mathrm{H} \alpha$ emission, as the nebular emission dominates the galaxy 
morphology (see Papaderos et al. 2002).

Top-middle: the total H I map. The contour levels are at 1, 2, $4,8, \ldots \times N_{\mathrm{HI}}(3 \sigma)$, where $N_{\mathrm{HI}}(3 \sigma)$ is the pseudo- $3 \sigma$ contour, calculated following Verheijen \& Sancisi (2001). The value of $N_{\mathrm{HI}}(3 \sigma)$ is given in the note. The cross shows the optical center. The ellipse shows the beam.

Bottom-middle: the HI surface density profile, derived by azimuthally averaging over the entire HI disk (black line) and over the approaching and receding sides separately (filled and open circles, respectively). In UGC 6541 and UGCA 290, $\mathrm{HI}$ emission is detected only on one side of the galaxy, thus the HI surface density profile was derived using the optical orientation parameters and averaging over a single side.

Top-right: the H I velocity field. Light and dark shading indicate approaching and receding velocities, respectively. The thick, black line shows the systemic velocity. The velocity interval between approaching (black) and receding (white) contours is given in the note. The cross shows the optical center, while the circle shows the kinematic center. The dashed line indicates the kinematic position angle. The ellipse shows the beam.

Bottom-right: Position-Velocity diagram taken through the kinematic center and along the kinematic major axis. Contours are at $-3,-1.5$ (dashed) $1.5,3,6,12, \ldots \times \sigma$. The value of $\sigma$ is given in Table B.1. The vertical and horizontal lines show the kinematic center and the systemic velocity, respectively. For galaxies with a regularly rotating H I disk, squares show the rotation curve as derived in Sect. 5.1, projected along the line of sight. For galaxies with a kinematically disturbed H I disk, arrows show the estimated value of $V_{\text {rot }}$, projected along the line of sight. 

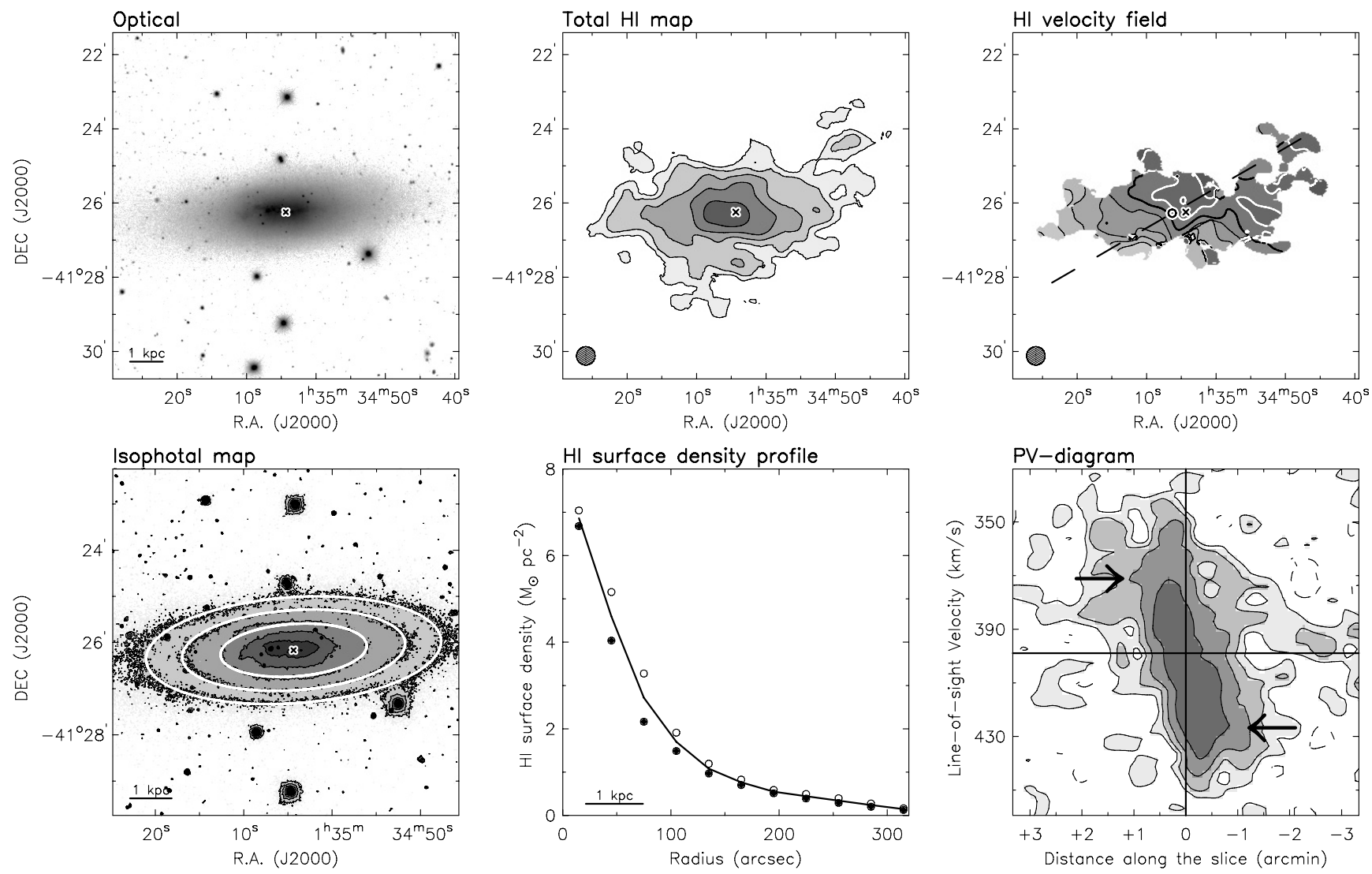

Fig. C.1. Contours: $\mu_{\text {out }}=24.5 \mathrm{R} \mathrm{mag} \operatorname{arcsec}^{-2} ; N_{\mathrm{HI}}(3 \sigma)=1.1 \times 10^{20}$ atoms cm${ }^{-2} ; V_{\text {los }}=398 \pm 10 \mathrm{~km} \mathrm{~s}^{-1}$.

NGC 1569
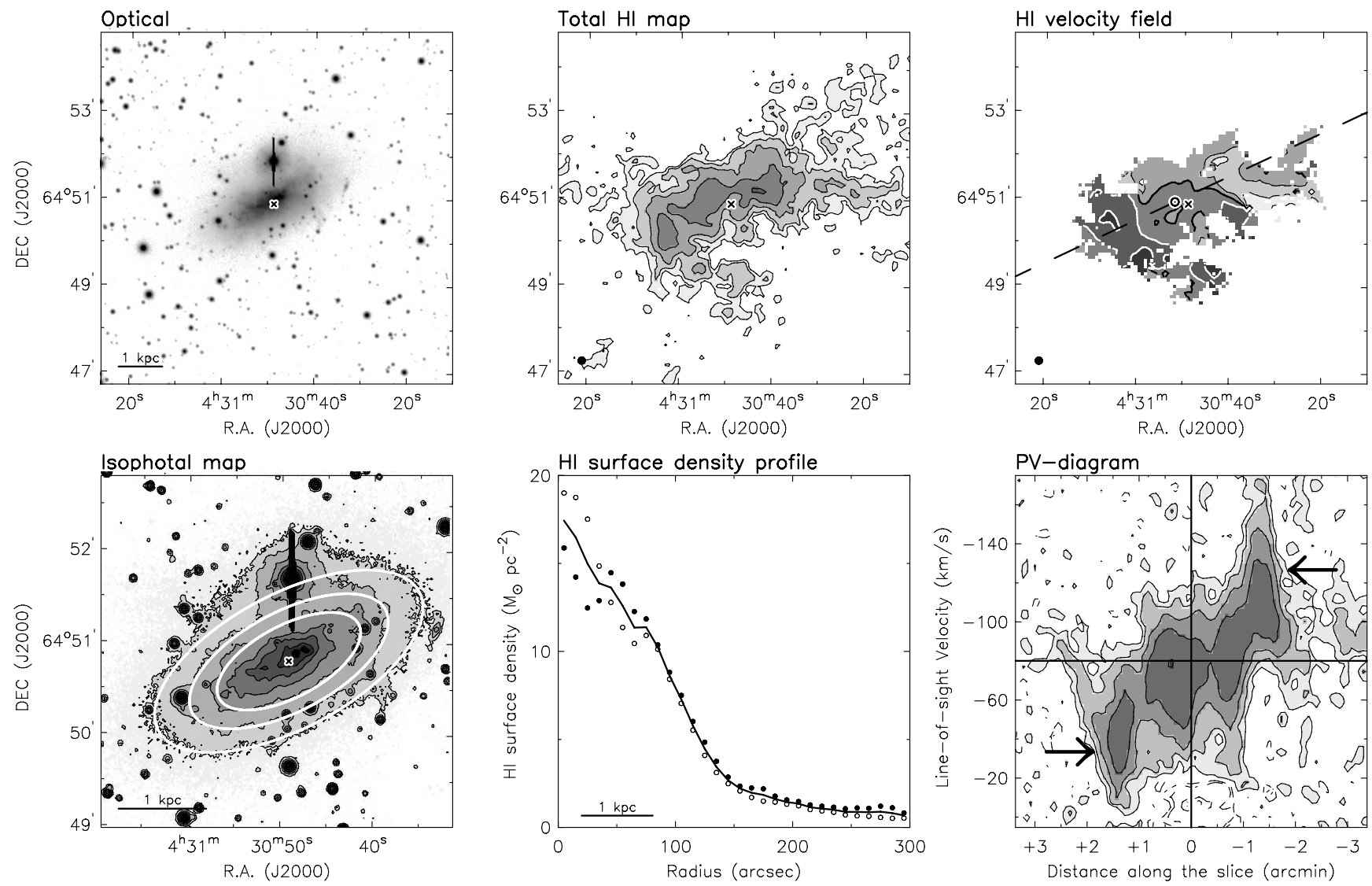

Fig. C.2. Contours: $\mu_{\text {out }}=24 \mathrm{~V} \mathrm{mag} \operatorname{arcsec}^{-2} ; N_{\mathrm{HI}}(3 \sigma)=4.3 \times 10^{20}$ atoms cm${ }^{-2} ; V_{\mathrm{los}}=-80 \pm 20 \mathrm{~km} \mathrm{~s}^{-1}$. 
F. Lelli et al.: Dynamics of starbursting dwarf galaxies. III.

NGC 1705
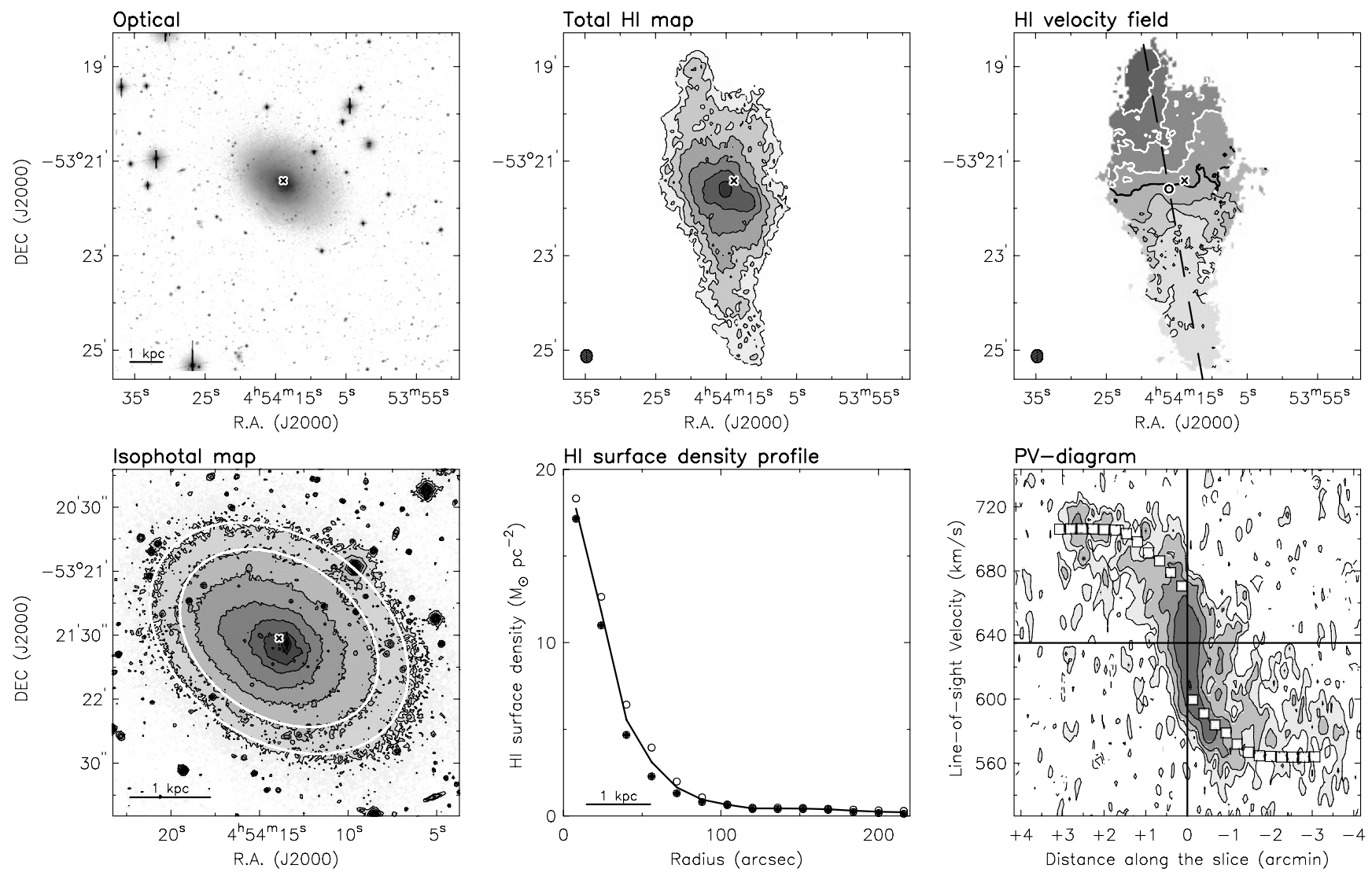

Fig. C.3. Contours: $\mu_{\text {out }}=25.5 \mathrm{R} \mathrm{mag} \operatorname{arcsec}^{-2} ; N_{\mathrm{HI}}(3 \sigma)=1.1 \times 10^{20}$ atoms $\mathrm{cm}^{-2} ; V_{\mathrm{los}}=635 \pm 20 \mathrm{~km} \mathrm{~s}^{-1}$.

NGC 2366
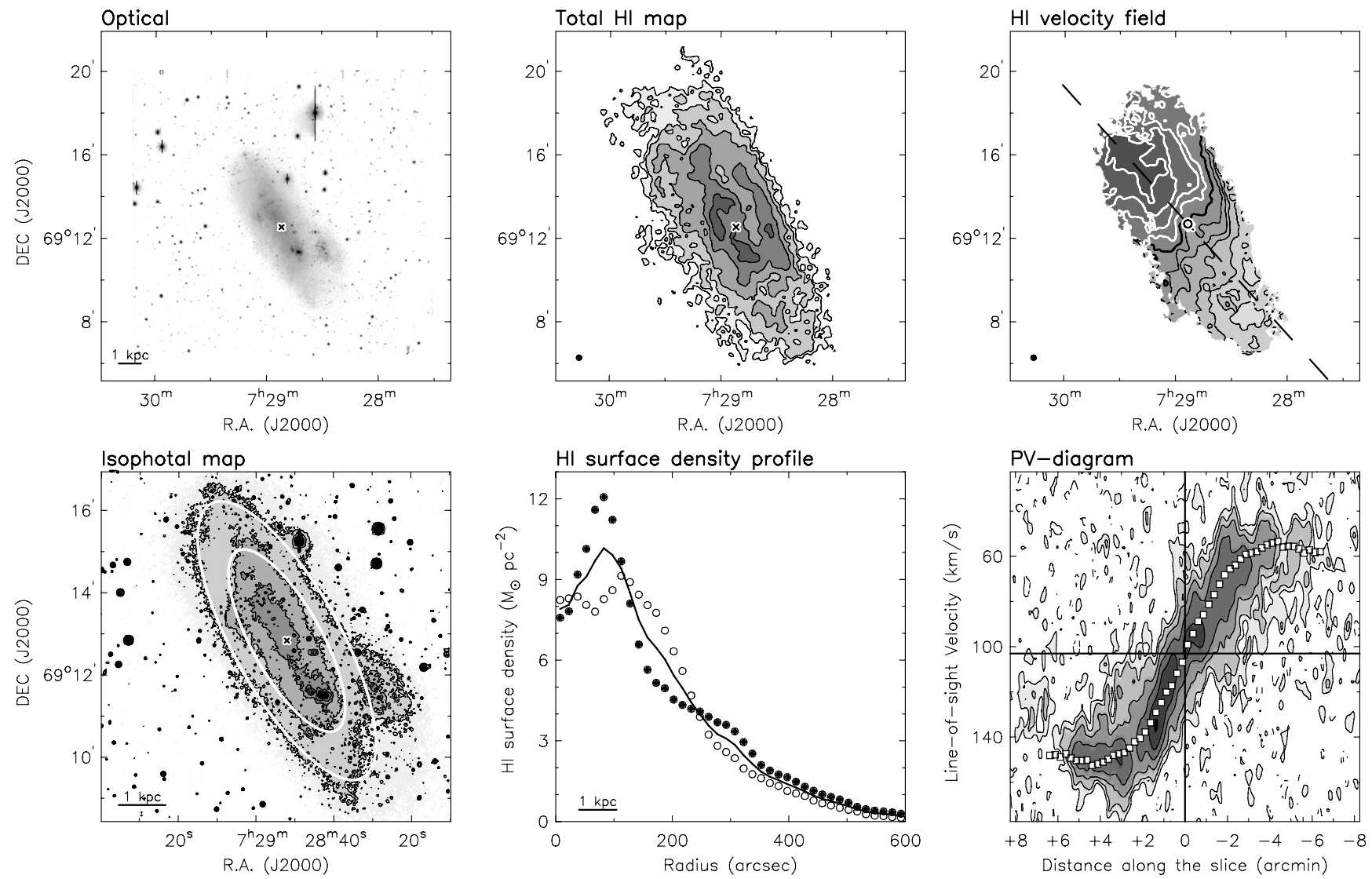

Fig. C.4. Contours: $\mu_{\text {out }}=24.5 \mathrm{~V} \mathrm{mag} \operatorname{arcsec}^{-2} ; N_{\mathrm{HI}}(3 \sigma)=2.3 \times 10^{20}$ atoms cm ${ }^{-2} ; V_{\text {los }}=103 \pm 10 \mathrm{~km} \mathrm{~s}^{-1}$. 
A\&A 566, A71 (2014)

NGC 4068
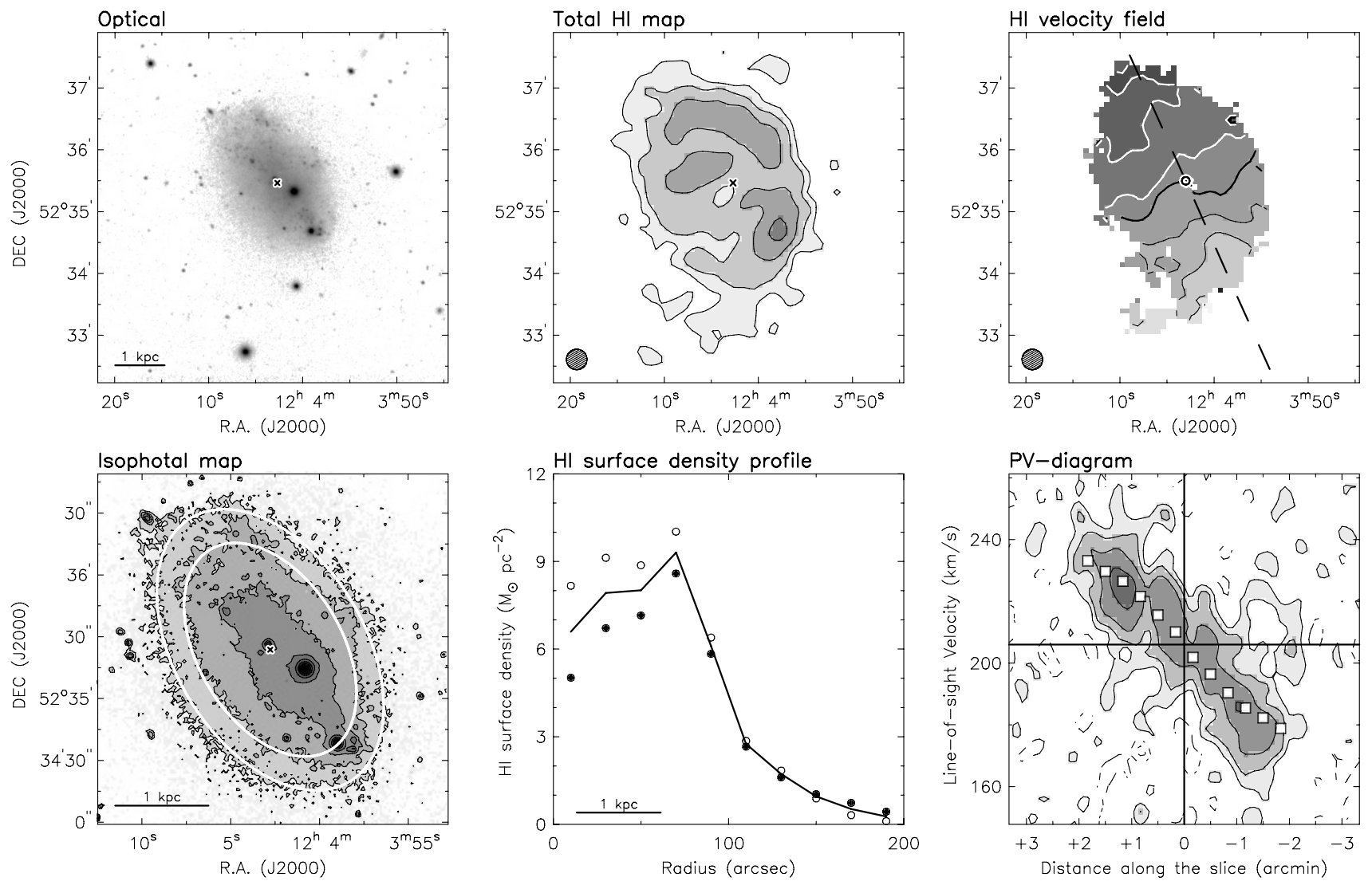

Fig. C.5. Contours: $\mu_{\text {out }}=24.5 \mathrm{R} \mathrm{mag} \operatorname{arcsec}^{-2} ; N_{\mathrm{HI}}(3 \sigma)=3.6 \times 10^{20}$ atoms cm${ }^{-2} ; V_{\text {los }}=206 \pm 10 \mathrm{~km} \mathrm{~s}^{-1}$.

NGC 4163
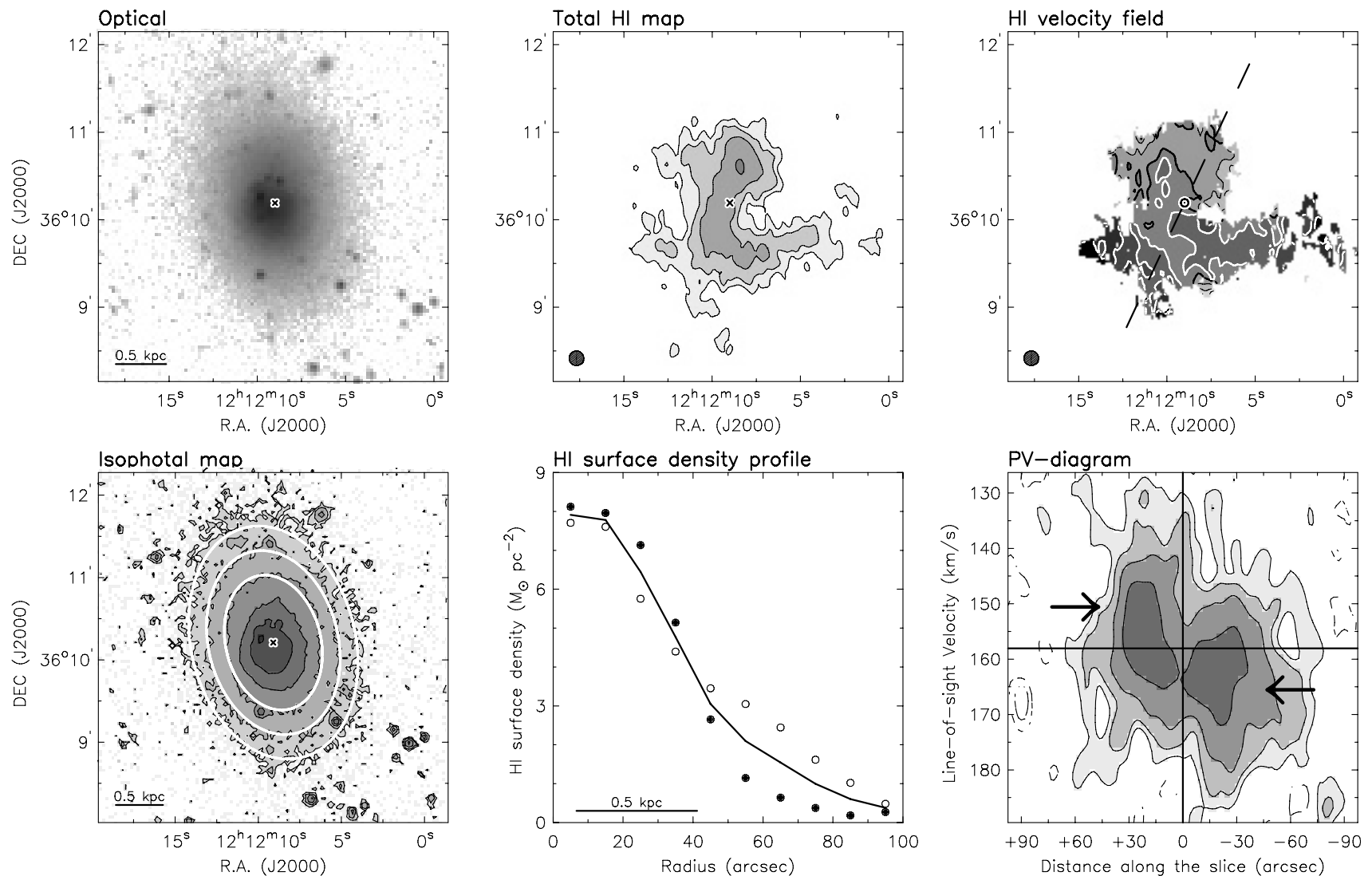

Fig. C.6. Contours: $\mu_{\text {out }}=26.5 \mathrm{~V} \mathrm{mag} \operatorname{arcsec}^{-2} ; N_{\mathrm{HI}}(3 \sigma)=2.7 \times 10^{20}$ atoms cm${ }^{-2} ; V_{\text {los }}=158 \pm 5 \mathrm{~km} \mathrm{~s}^{-1}$. 
F. Lelli et al.: Dynamics of starbursting dwarf galaxies. III.

NGC 4214
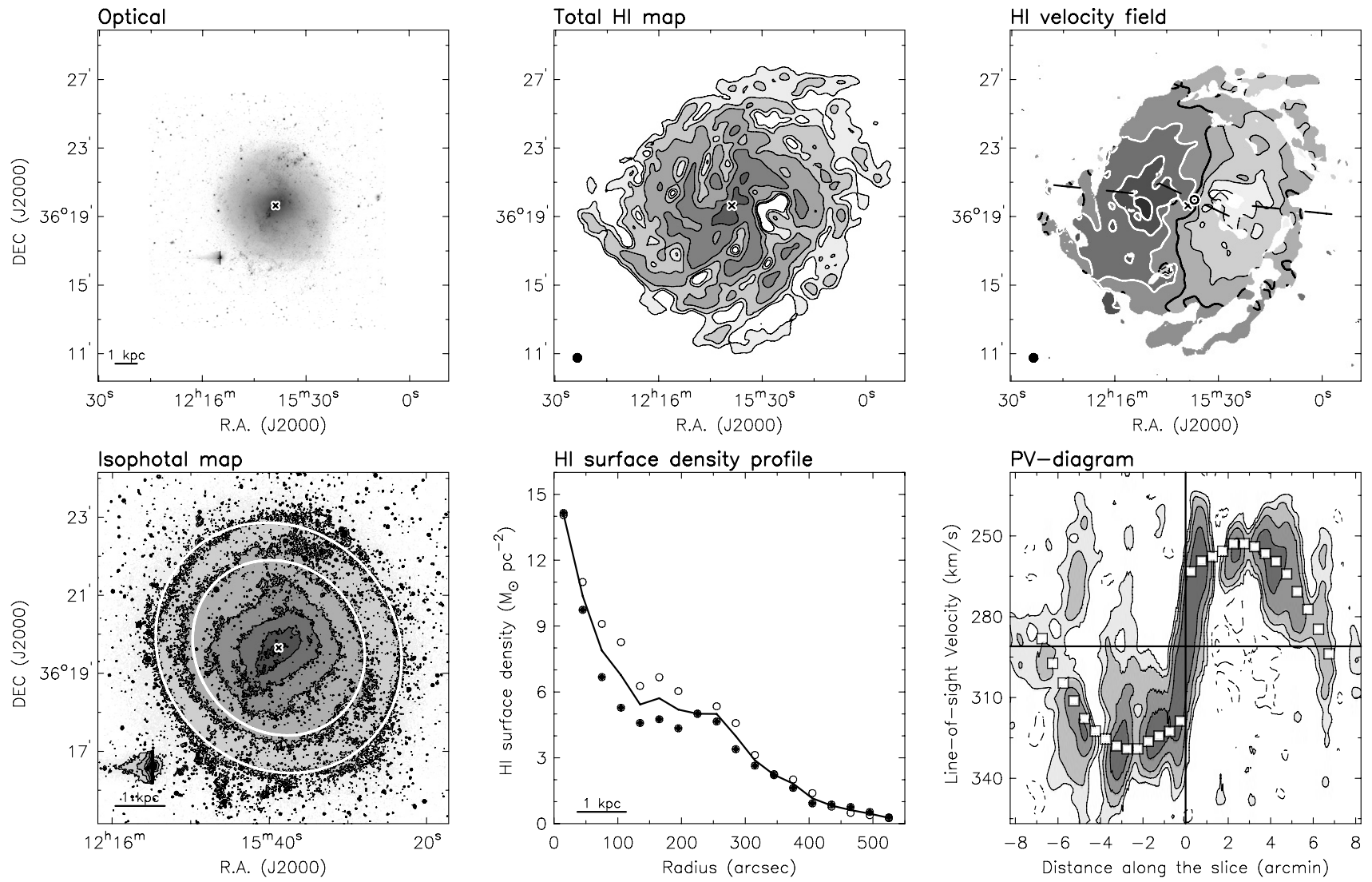

Fig. C.7. Contours: $\mu_{\text {out }}=24.5 \mathrm{~V} \mathrm{mag} \operatorname{arcsec}^{-2} ; N_{\mathrm{HI}}(3 \sigma)=1.2 \times 10^{20}$ atoms $\mathrm{cm}^{-2} ; V_{\text {los }}=291 \pm 15 \mathrm{~km} \mathrm{~s}^{-1}$.

NGC 4449
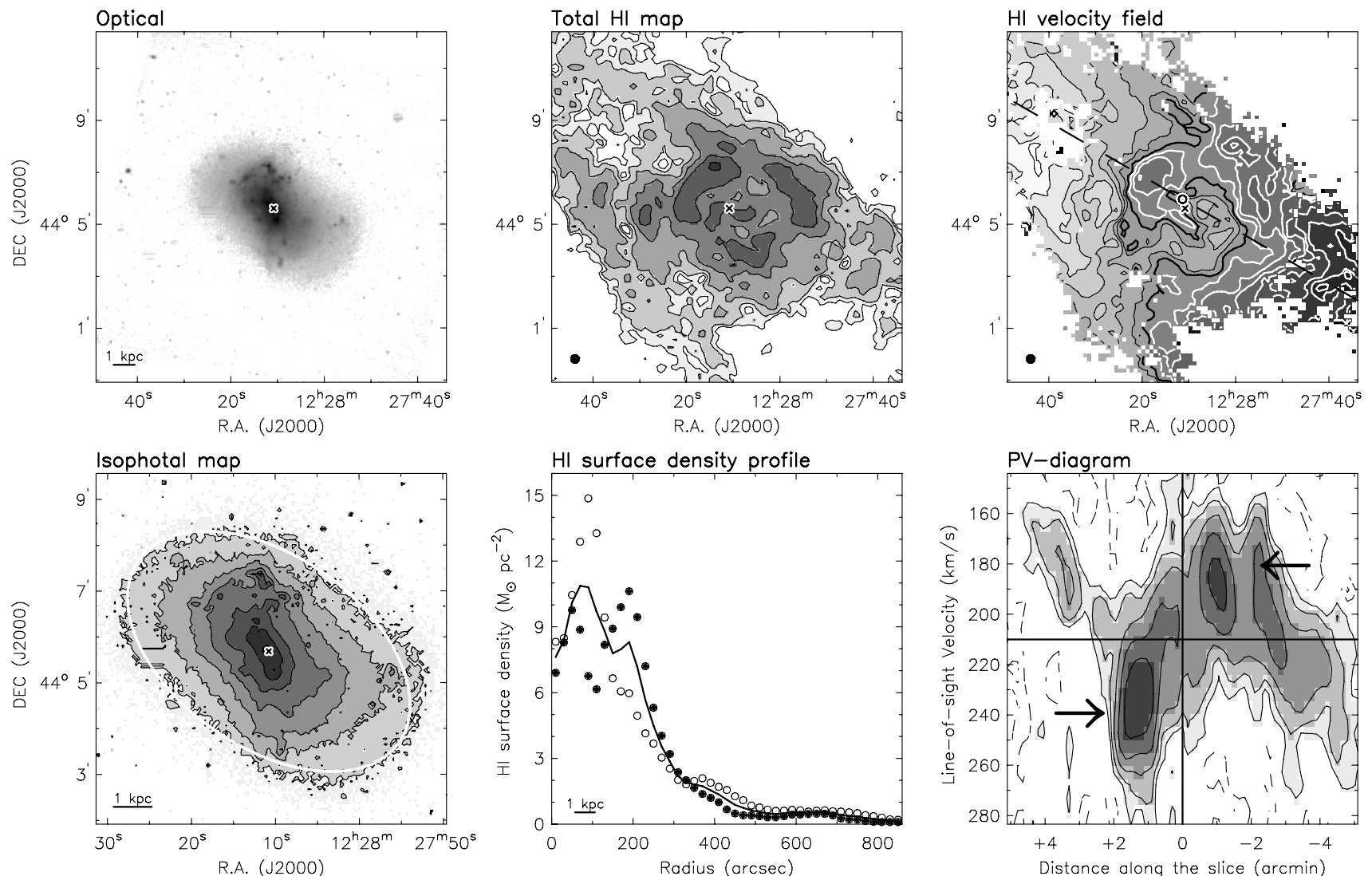

Fig. C.8. Contours: $\mu_{\text {out }}=25 \mathrm{~V} \mathrm{mag} \operatorname{arcsec}^{-2} ; N_{\mathrm{HI}}(3 \sigma)=1.4 \times 10^{20}$ atoms cm${ }^{-2} ; V_{\mathrm{los}}=210 \pm 10 \mathrm{~km} \mathrm{~s}^{-1}$. 
A\&A 566, A71 (2014)

NGC 5253
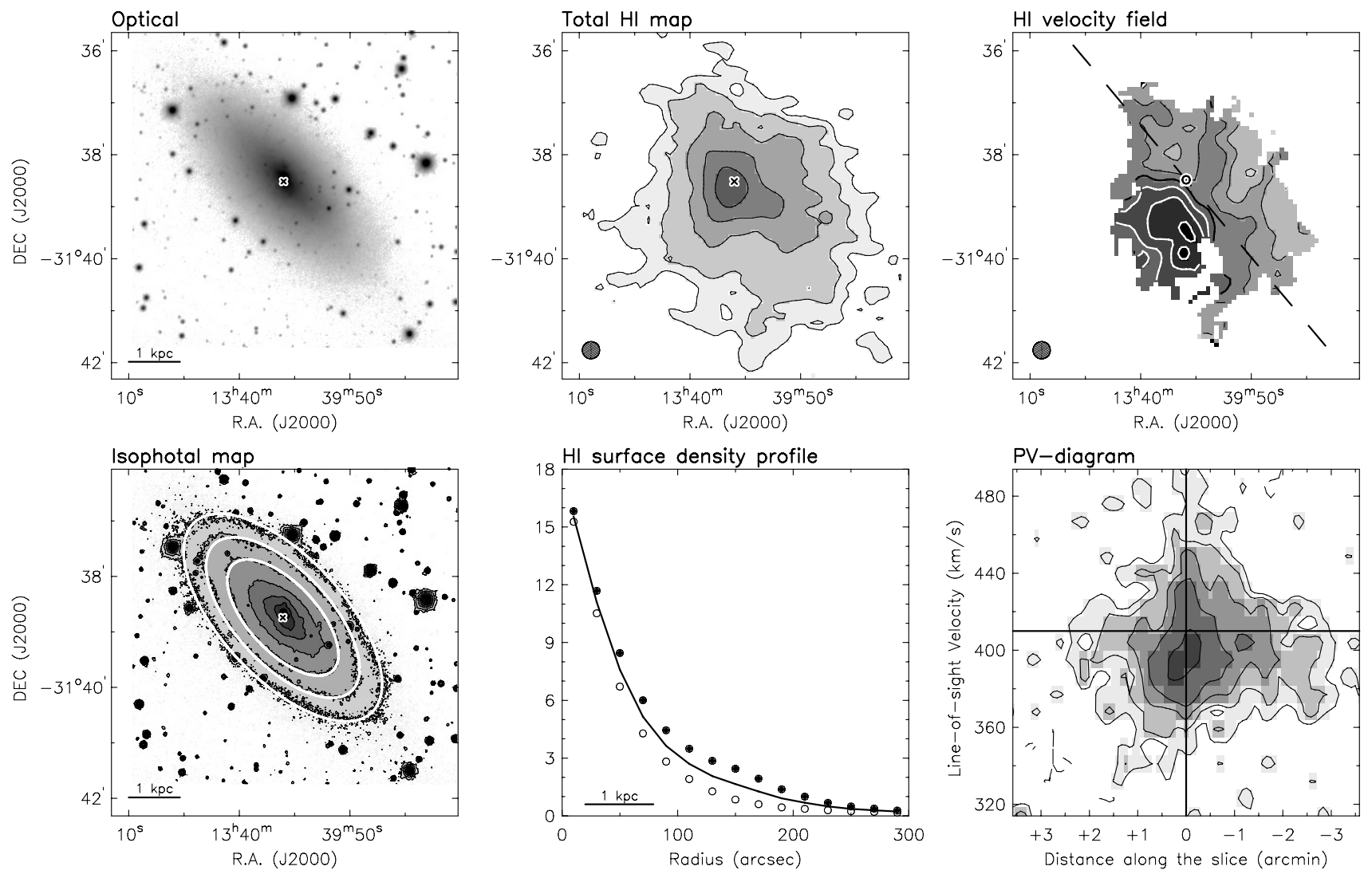

Fig. C.9. Contours: $\mu_{\text {out }}=24 \mathrm{R} \mathrm{mag} \operatorname{arcsec}^{-2} ; N_{\mathrm{HI}}(3 \sigma)=2.1 \times 10^{20}$ atoms cm${ }^{-2} ; V_{\mathrm{los}}=410 \pm 10 \mathrm{~km} \mathrm{~s}^{-1}$.
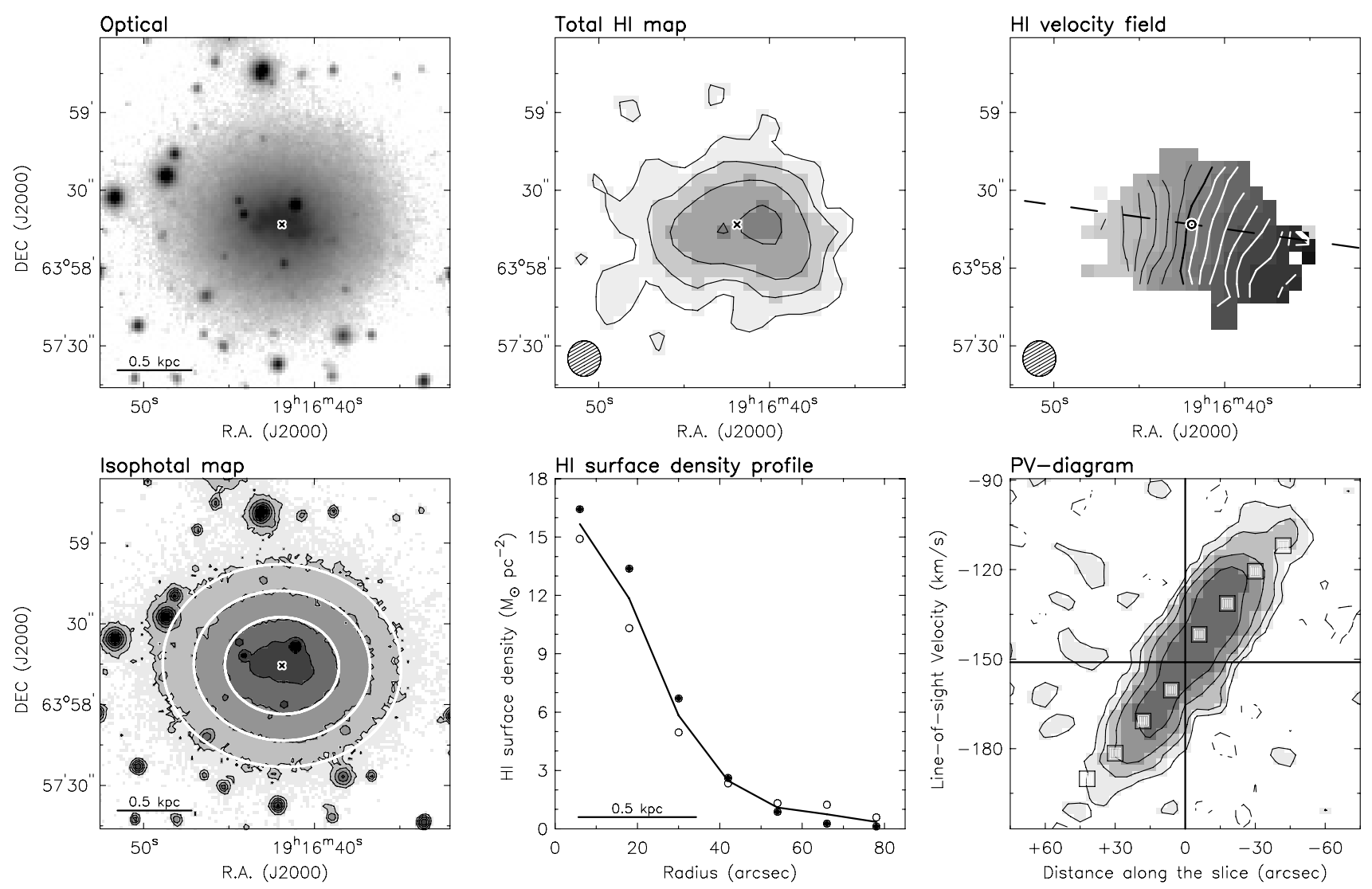

Fig. C.10. Contours: $\mu_{\text {out }}=24.5 \mathrm{R} \mathrm{mag} \operatorname{arcsec}^{-2} ; N_{\mathrm{HI}}(3 \sigma)=3.4 \times 10^{20}$ atoms cm${ }^{-2} ; V_{\mathrm{los}}=-151 \pm 5 \mathrm{~km} \mathrm{~s}^{-1}$. 
F. Lelli et al.: Dynamics of starbursting dwarf galaxies. III.

UGC 4483
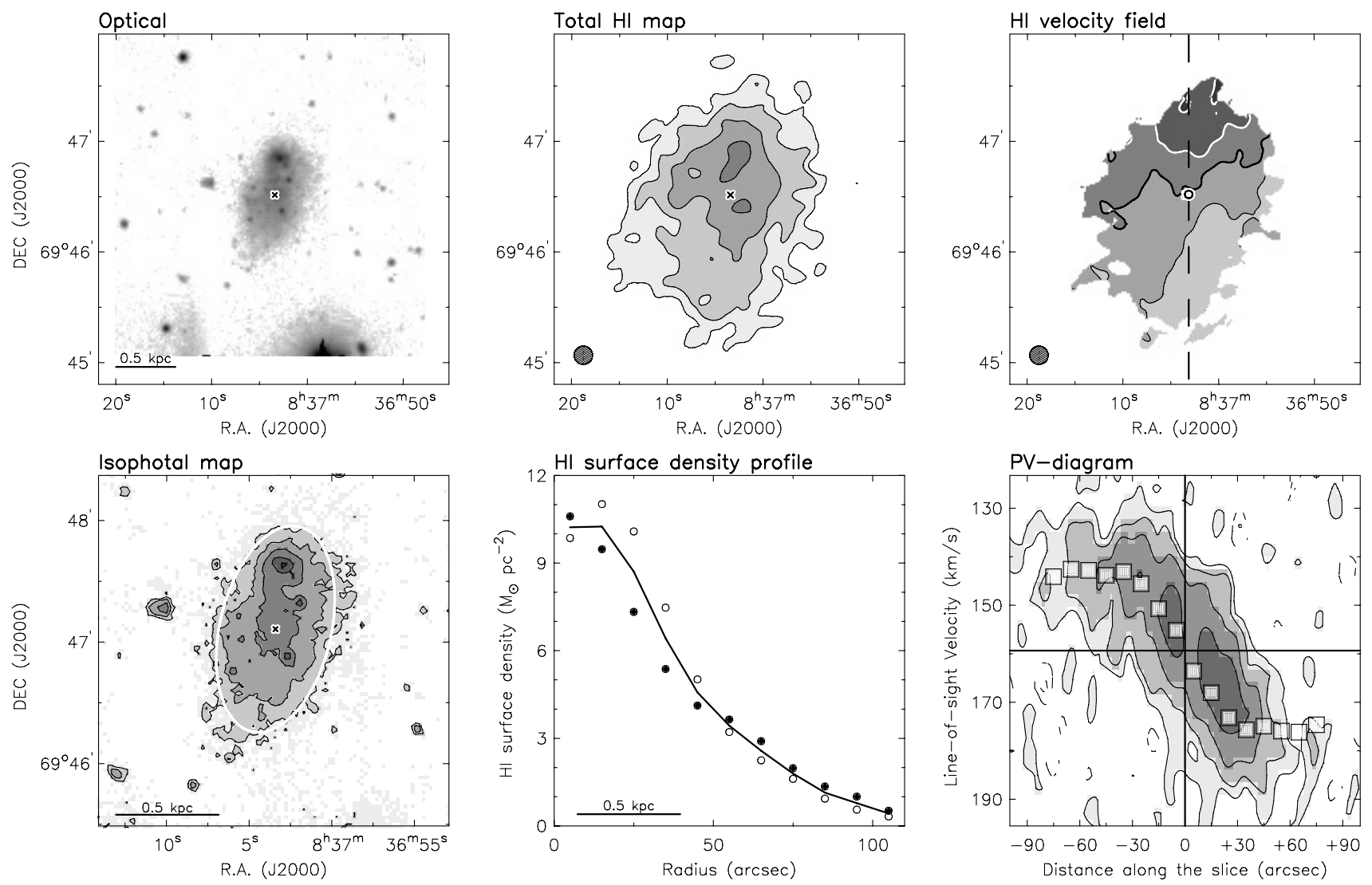

Fig. C.11. Contours: $\mu_{\text {out }}=25 \mathrm{R}$ mag $\operatorname{arcsec}^{-2} ; N_{\mathrm{HI}}(3 \sigma)=3.4 \times 10^{20}$ atoms cm ${ }^{-2} ; V_{\mathrm{los}}=158 \pm 10 \mathrm{~km} \mathrm{~s}^{-1}$.

UGC 6456
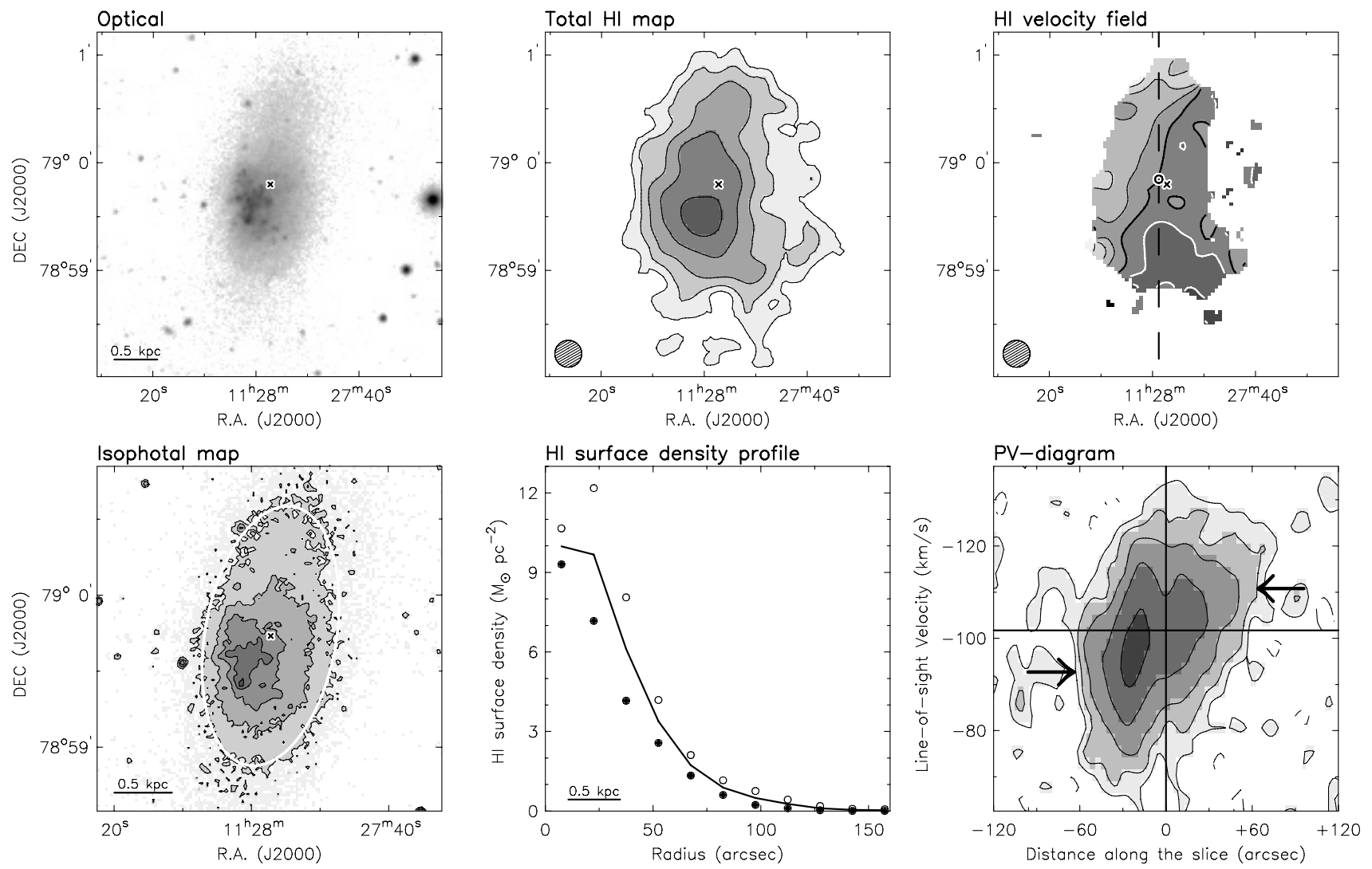

Fig. C.12. Contours: $\mu_{\text {out }}=25 \mathrm{R} \mathrm{mag} \operatorname{arcsec}^{-2} ; N_{\mathrm{HI}}(3 \sigma)=1.8 \times 10^{20}$ atoms cm${ }^{-2} ; V_{\mathrm{los}}=-102 \pm 5 \mathrm{~km} \mathrm{~s}^{-1}$. 
A\&A 566, A71 (2014)

UGC 6541
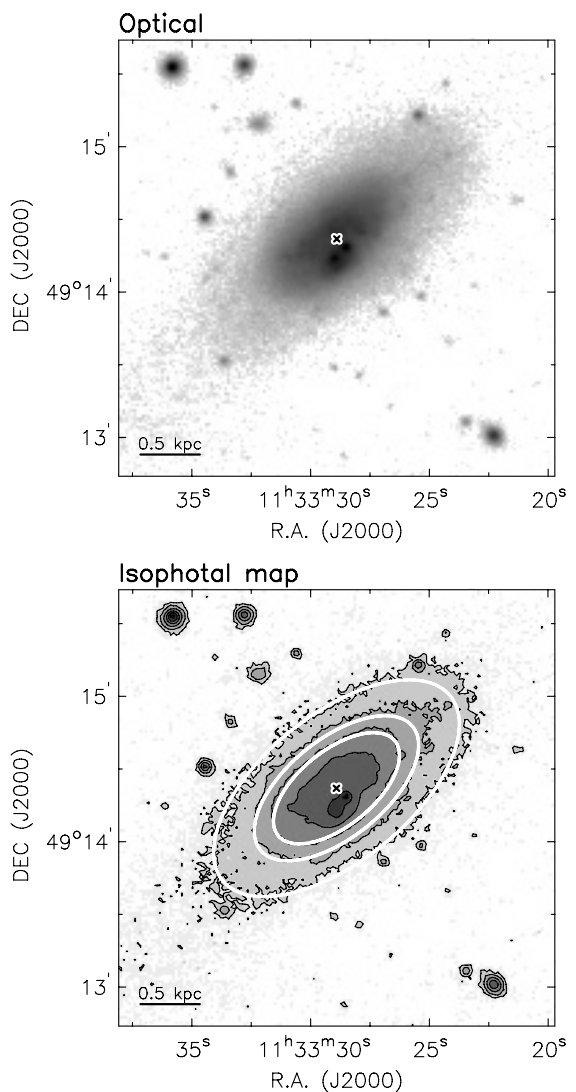
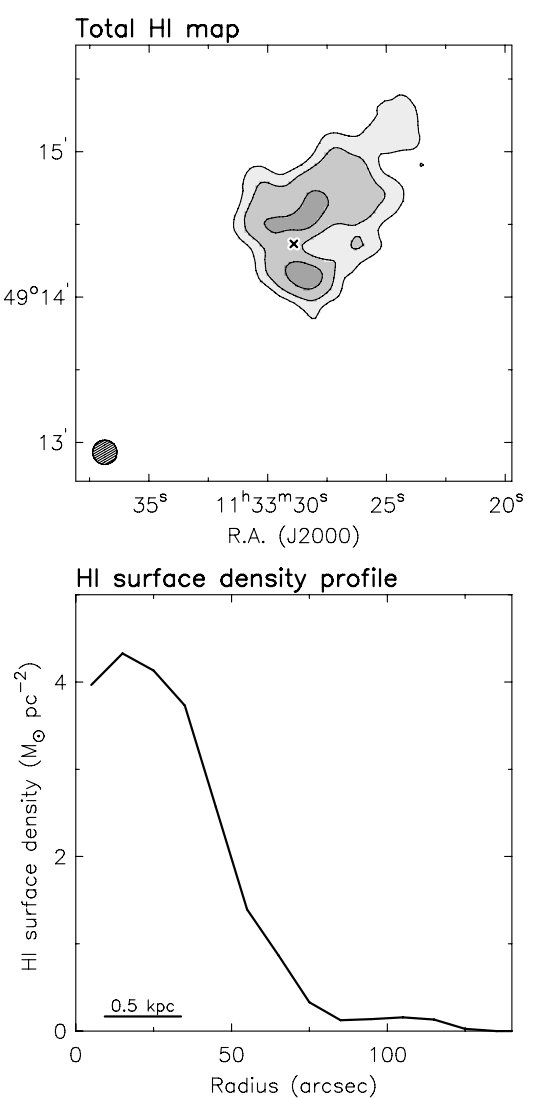
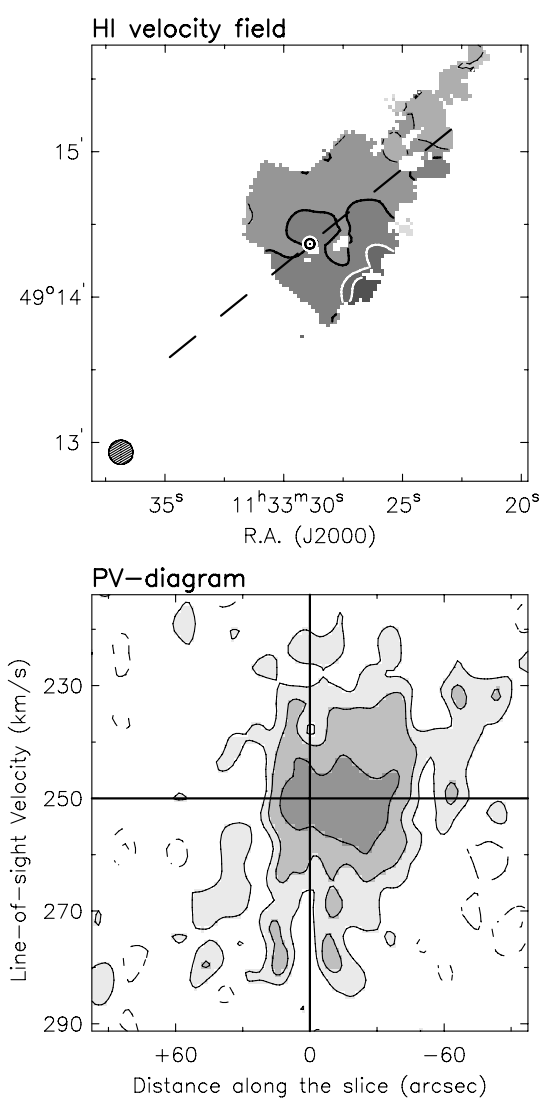

Fig. C.13. Contours: $\mu_{\text {out }}=25.5 \mathrm{R} \mathrm{mag} \operatorname{arcsec}^{-2} ; N_{\mathrm{HI}}(3 \sigma)=3.4 \times 10^{20}$ atoms cm${ }^{-2} ; V_{\mathrm{los}}=250 \pm 10 \mathrm{~km} \mathrm{~s}^{-1}$.

UGC 9128
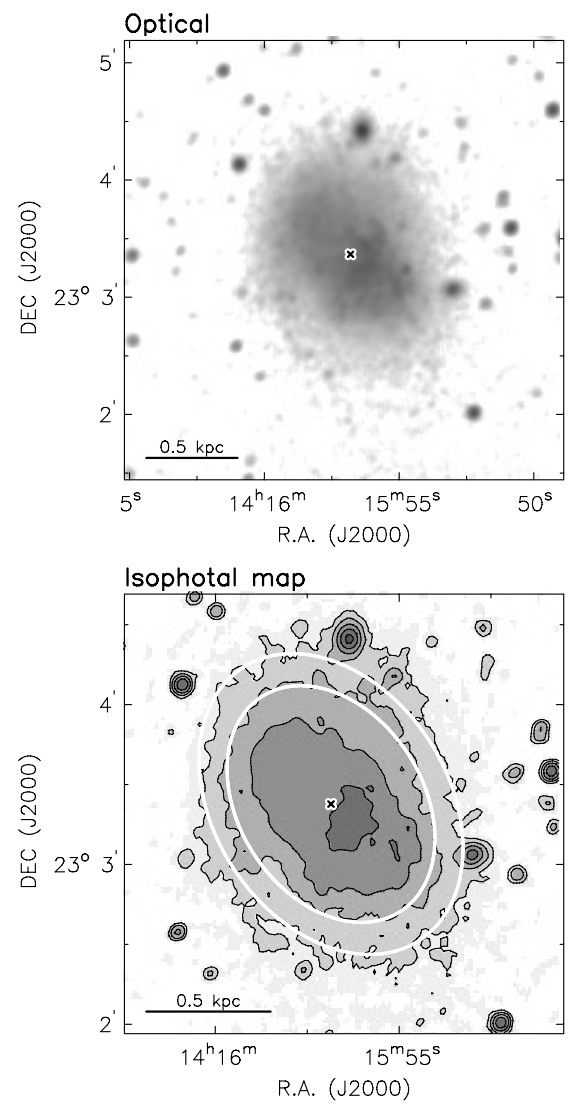

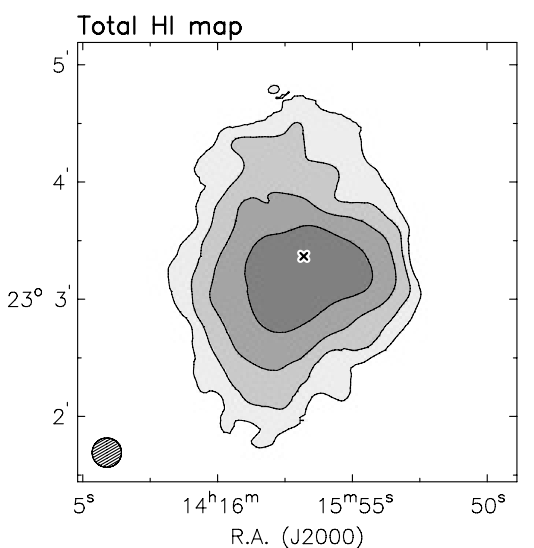

HI surface density profile

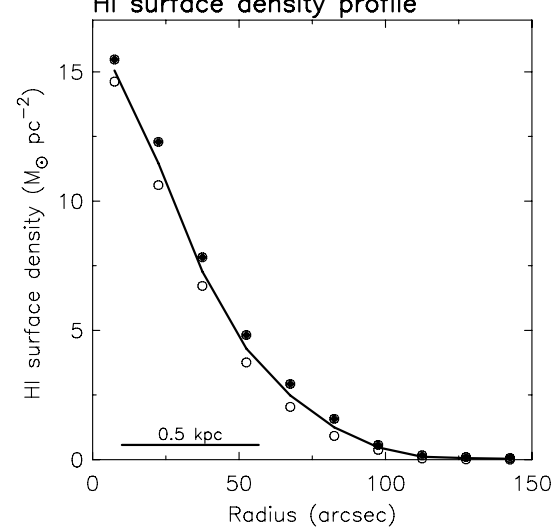

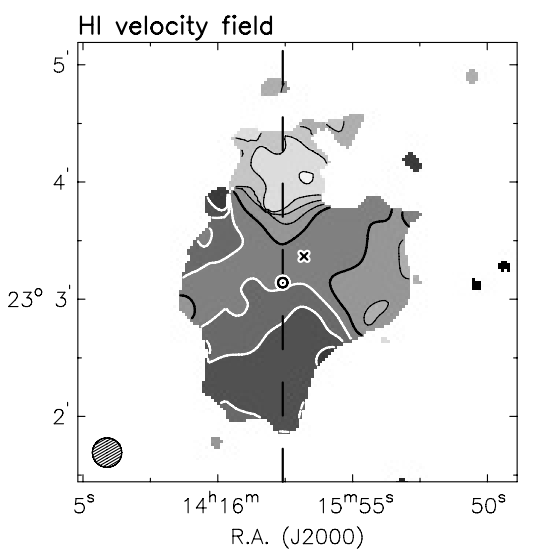

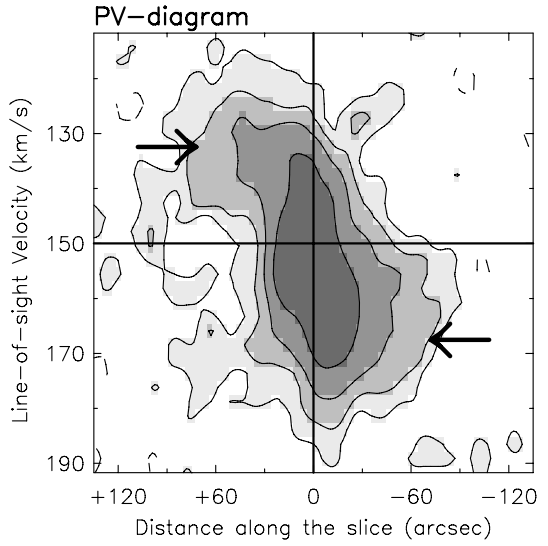

Fig. C.14. Contours: $\mu_{\text {out }}=26.5 \mathrm{~V} \mathrm{mag} \operatorname{arcsec}^{-2} ; N_{\mathrm{HI}}(3 \sigma)=2.0 \times 10^{20}$ atoms cm $\mathrm{cm}^{-2} ; V_{\text {los }}=150 \pm 5 \mathrm{~km} \mathrm{~s}^{-1}$. 
F. Lelli et al.: Dynamics of starbursting dwarf galaxies. III.

UGCA 290
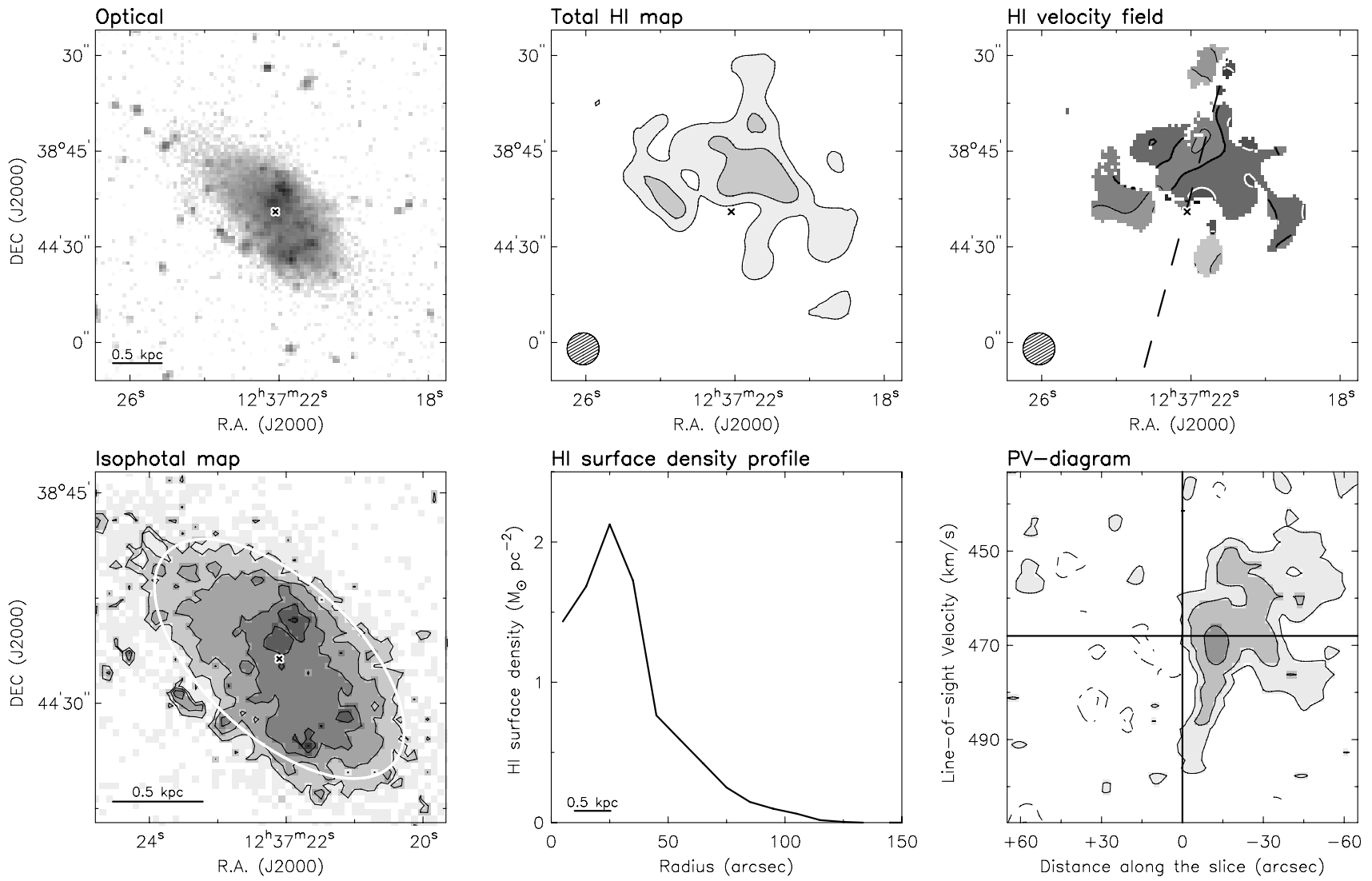

Fig. C.15. Contours: $\mu_{\text {out }}=25 \mathrm{R} \mathrm{mag} \operatorname{arcsec}^{-2} ; N_{\mathrm{HI}}(3 \sigma)=3.1 \times 10^{20}$ atoms cm${ }^{-2} ; V_{\mathrm{los}}=468 \pm 5 \mathrm{~km} \mathrm{~s}^{-1}$.

I Zw 18
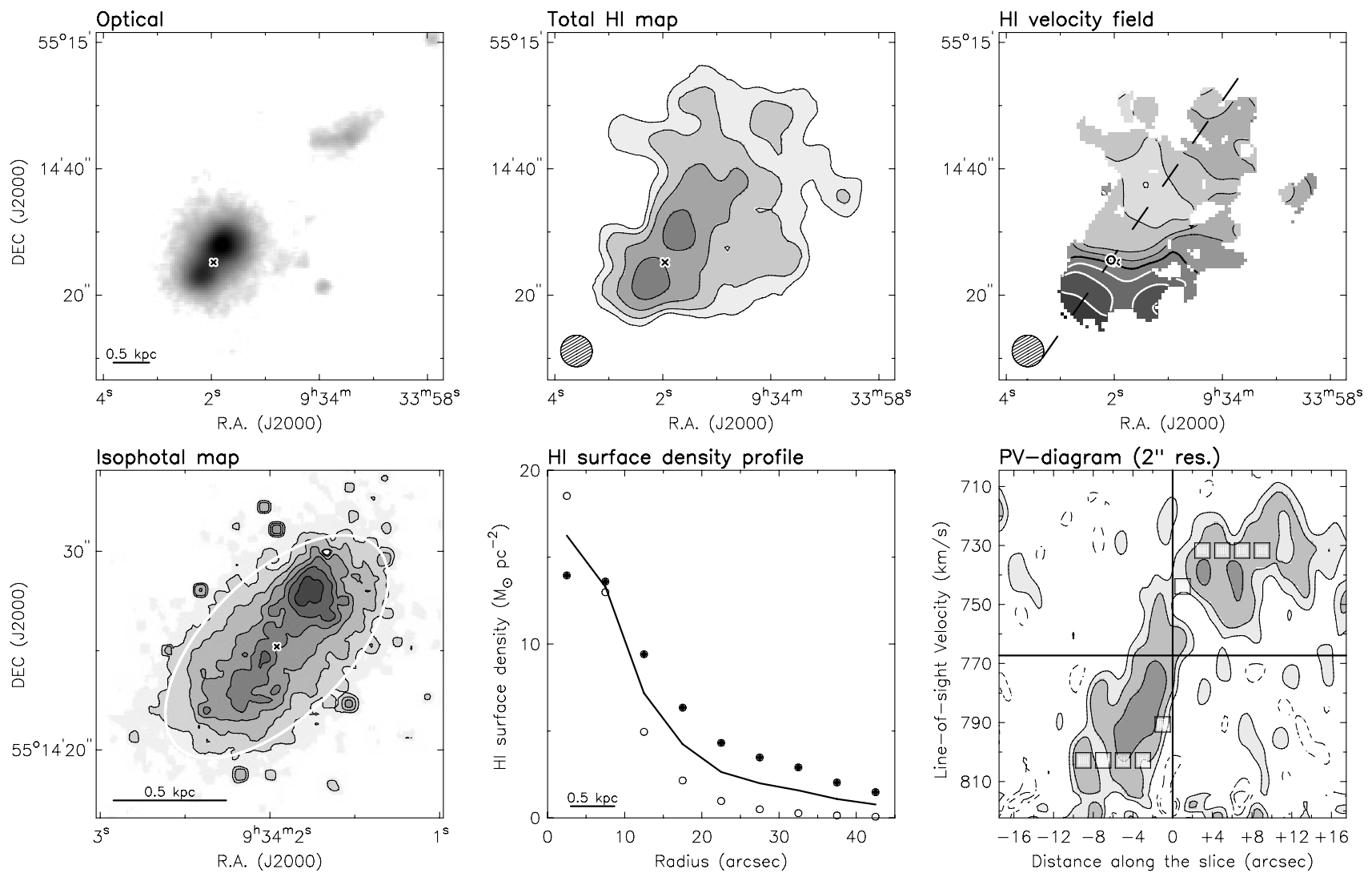

Fig. C.16. Contours: $\mu_{\text {out }}=24 \mathrm{R} \mathrm{mag} \operatorname{arcsec}^{-2} ; N_{\mathrm{HI}}(3 \sigma)=6.3 \times 10^{20}$ atoms cm $\mathrm{cm}^{-2} ; V_{\text {los }}=767 \pm 10 \mathrm{~km} \mathrm{~s}^{-1}$. 
A\&A 566, A71 (2014)

I Zw 36
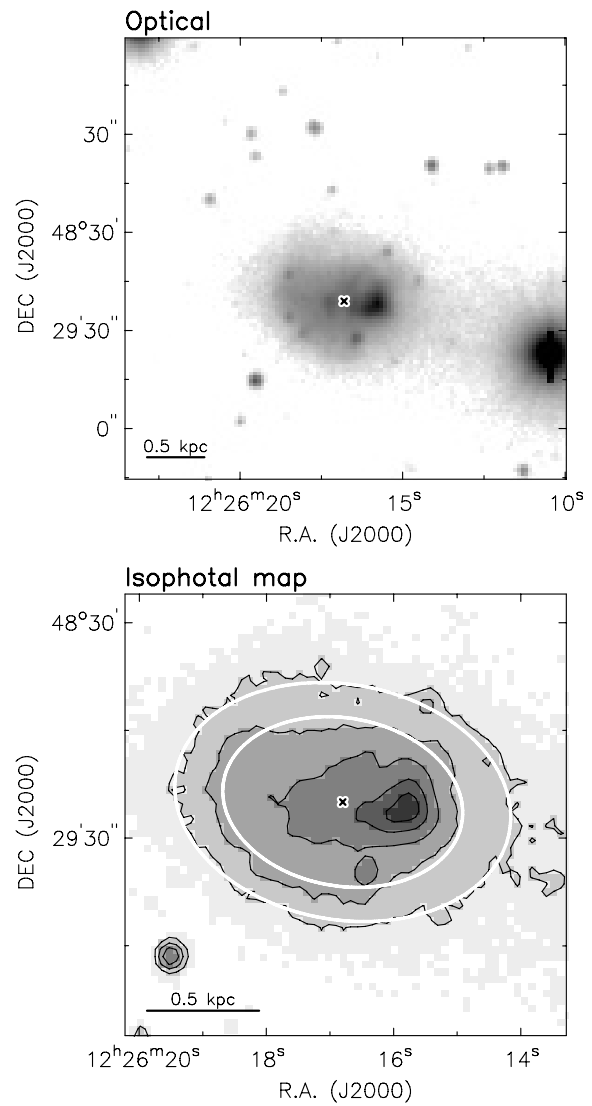
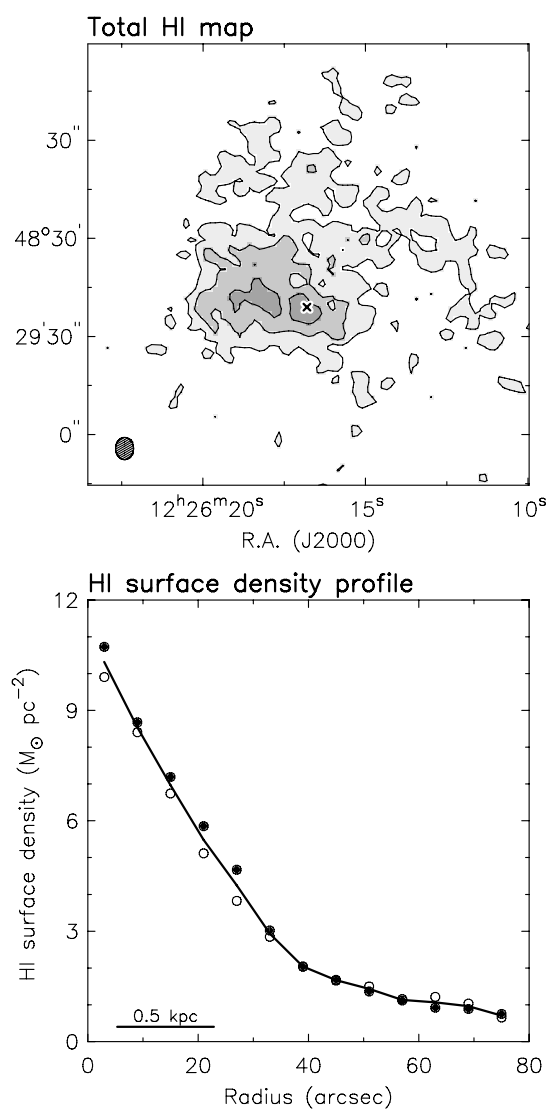
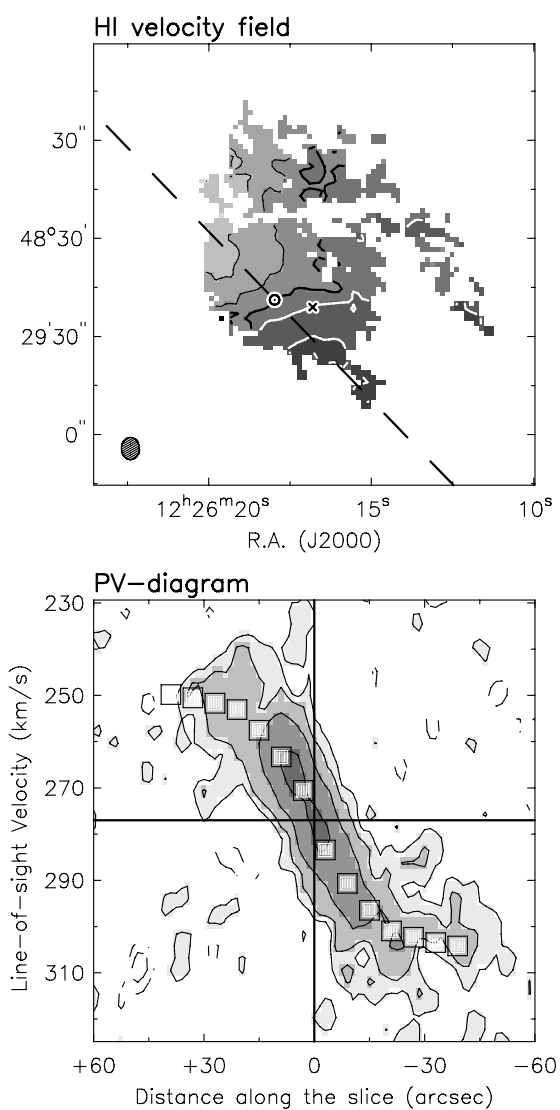

Fig. C.17. Contours: $\mu_{\text {out }}=24.5 \mathrm{R} \mathrm{mag} \operatorname{arcsec}^{-2} ; N_{\mathrm{HI}}(3 \sigma)=7.4 \times 10^{20}$ atoms cm${ }^{-2} ; V_{\mathrm{los}}=277 \pm 10 \mathrm{~km} \mathrm{~s}^{-1}$.

SBS $1415+437$
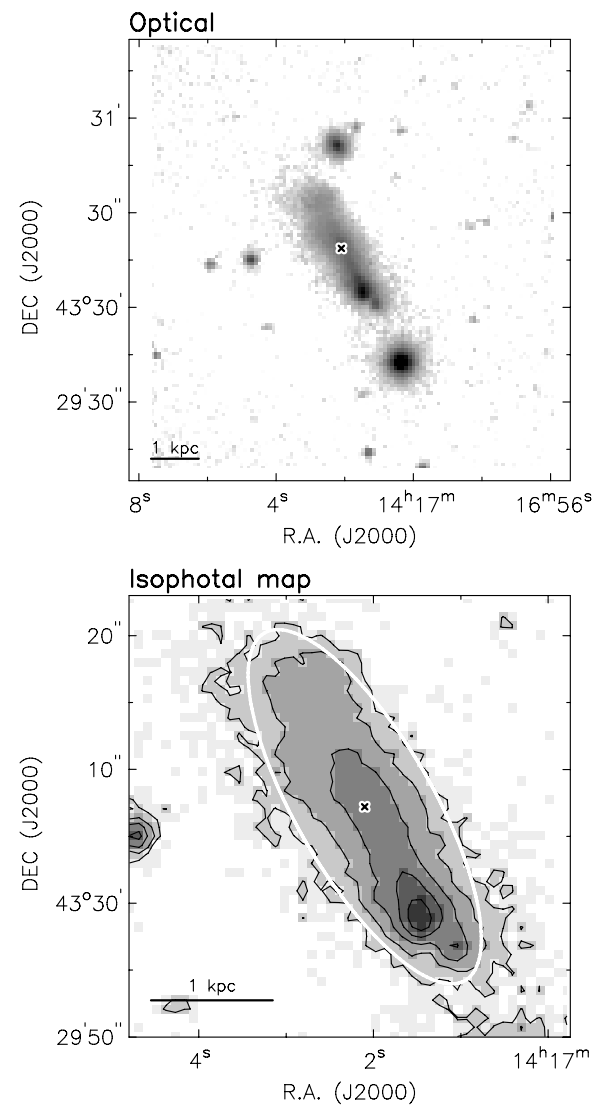
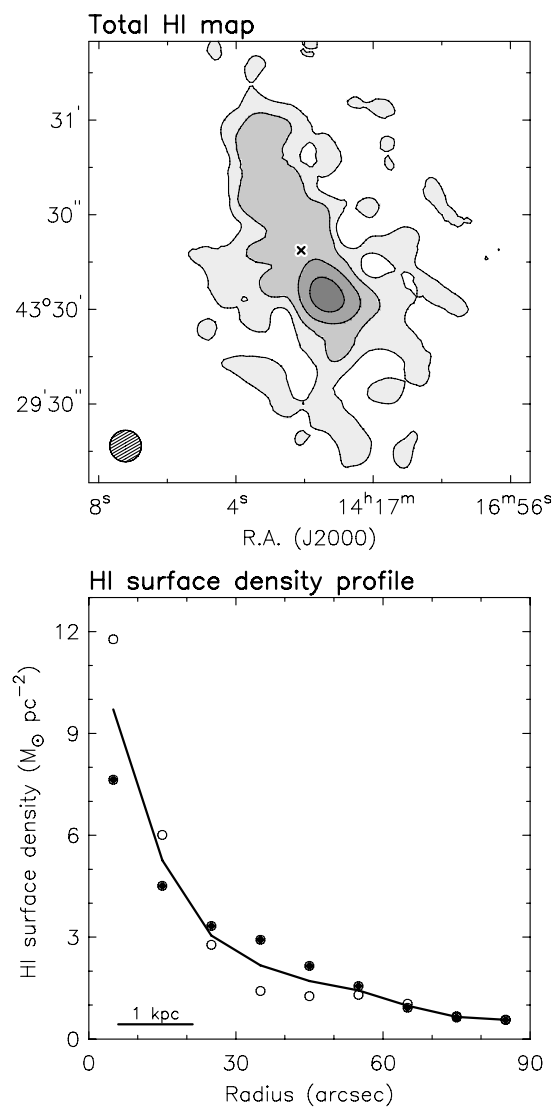
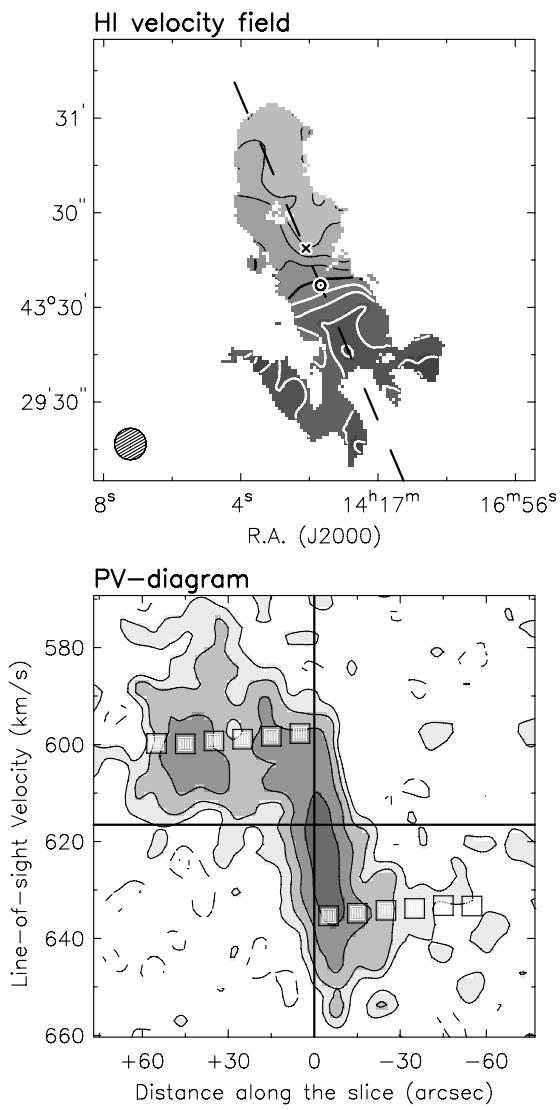

Fig. C.18. Contours: $\mu_{\mathrm{out}}=24.5 \mathrm{R} \mathrm{mag} \operatorname{arcsec}^{-2} ; N_{\mathrm{HI}}(3 \sigma)=3.7 \times 10^{20}$ atoms cm${ }^{-2} ; V_{\mathrm{los}}=616 \pm 5 \mathrm{~km} \mathrm{~s}^{-1}$. 